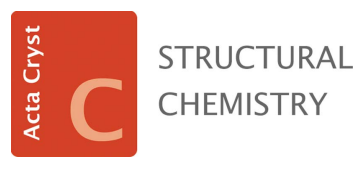

ISSN 2053-2296
Received 23 April 2019

Accepted 30 July 2019

Edited by E. Y. Cheung, Amgen Inc., USA

Keywords: alcohol; hydrogen bond; disorder topological chirality; crystal structure; triphenylmethanol.

CCDC references: 1944593; 1944592

Supporting information: this article has supporting information at journals.iucr.org/c

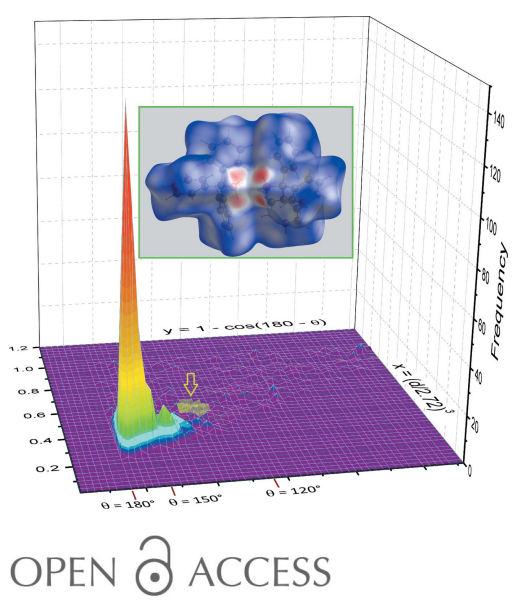

\section{Deciphering the hydrogen-bonding scheme in the crystal structure of triphenylmethanol: a tribute to George Ferguson and co-workers}

\author{
Tomasa Rodríguez Tzompantzi, ${ }^{a}$ Aldo Guillermo Amaro Hernández, ${ }^{a}$ Rosa Luisa \\ Meza-León ${ }^{a}$ and Sylvain Bernès ${ }^{b_{*}}$
}

${ }^{a}$ Facultad de Ciencias Químicas, Benemérita Universidad Autónoma de Puebla, Av. San Claudio y 18 Sur, 72570 Puebla,
Pue., Mexico, and ${ }^{\mathbf{b}}$ Instituto de Física, Benemérita Universidad Autónoma de Puebla, Av. San Claudio y 18 Sur, 72570
Puebla, Pue., Mexico. *Correspondence e-mail: sylvain_bernes@hotmail.com

The crystal structure of triphenylmethanol, $\mathrm{C}_{19} \mathrm{H}_{16} \mathrm{O}$, has been redetermined using data collected at 295 and $153 \mathrm{~K}$, and is compared to the model published by Ferguson et al. over 25 years ago [Ferguson et al. (1992). Acta Cryst. C48, 1272-1275] and that published by Serrano-González et al., using neutron and X-ray diffraction data [Serrano-González et al. (1999). J. Phys. Chem. B, 103, 6215-6223]. As predicted by these authors, the hydroxy groups are involved in weak intermolecular hydrogen bonds in the crystal, forming tetrahedral tetramers based on the two independent molecules in the asymmetric unit, one of which is placed on the threefold symmetry axis of the $R \overline{3}$ space group. However, the reliable determination of the hydroxy $\mathrm{H}$-atom positions is difficult to achieve, for two reasons. Firstly, a positional disorder affects the full asymmetric unit, which is split over two sets of positions, with occupancy factors of $c a 0.74$ and 0.26 . Secondly, all hydroxy $\mathrm{H}$ atoms are further disordered, either by symmetry, or through a positional disorder in the case of parts placed in general positions. We show that the correct description of the hydrogen-bonding scheme is possible only if diffraction data are collected at low temperature. The prochiral character of the hydrogen-bonded tetrameric supramolecular clusters leads to enantiomorphic three-dimensional graphs in each tetramer. The crystal is thus a racemic mixture of ${ }^{\text {sup }} S$ and ${ }^{\text {sup }} R$ motifs, consistent with the centrosymmetric nature of the $R \overline{3}$ space group.

\section{Introduction}

The hydroxy group is known as one of the most efficient nodes for the formation of hydrogen bonds, as a consequence of the polarization of the $\mathrm{O}-\mathrm{H}$ bond, and also because it can behave both as donor and acceptor for building intra- or intermolecular bonds. In this context, the emblematic donoracceptor molecule is water, and many compounds have been crystallized as hydrates, in which the lattice water molecules contribute to a significant part of the crystal free energy (Batsanov, 2018); currently, almost $13 \%$ of the structures deposited in the Cambridge Structural Database are hydrates (CSD, Version 5.40, updated February 2019; Groom et al., 2016). The situation is a bit less favourable in the case of alcohols $(R \mathrm{O}-\mathrm{H})$, especially for tertiary alcohols having the hydroxy group surrounded by bulky hydrocarbon groups. For example, three hydrates for tert-butanol, $\left(\mathrm{CH}_{3}\right)_{3} \mathrm{COH}$, have been successfully characterized [namely the dihydrate and heptahydrate (Mootz \& Stäben, 1993), and the decahydrate (Dobrzycki, 2018)], while tri-tert-butylmethanol, $\left[\left(\mathrm{CH}_{3}\right)_{3} \mathrm{C}\right]_{3^{-}}$ $\mathrm{COH}$, has probably never been crystallized, although it has been studied in the solid state (Malarski, 1974). Although this 
Table 1

Experimental details.

For both determinations: $\mathrm{C}_{19} \mathrm{H}_{16} \mathrm{O}, M_{\mathrm{r}}=260.32$, trigonal, $R \overline{3}: H, Z=24$. Experiments were carried out with $\mathrm{Ag} K \alpha$ radiation $(\lambda=0.56083 \AA)$ using a Stoe Stadivari diffractometer. Absorption was corrected for by multi-scan methods ( $X$-AREA; Stoe \& Cie, 2018). Refinement was on 483 parameters. $\mathrm{H}$-atom parameters were constrained.

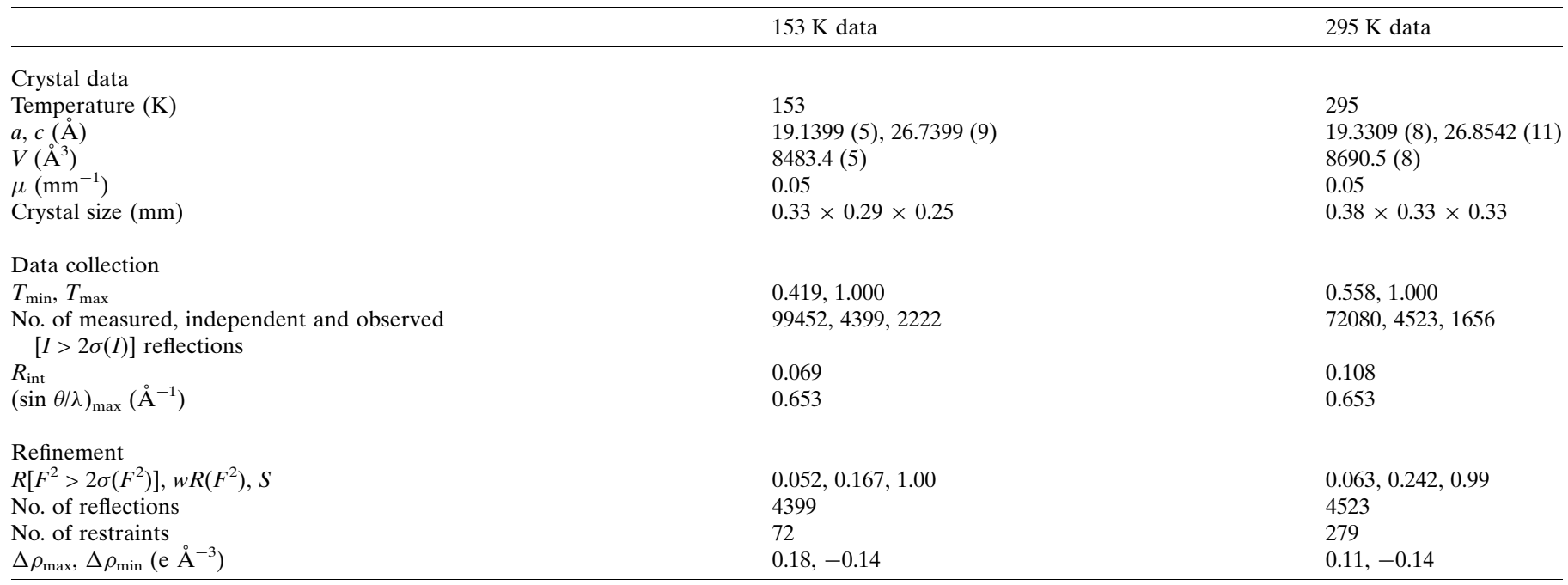

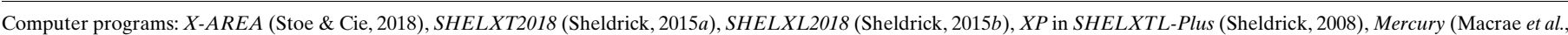
2008), OLEX2 (Dolomanov et al., 2009) and publCIF (Westrip, 2010).

molecule is stable, it is not able to form stabilizing intermolecular $\mathrm{O}-\mathrm{H}$... O hydrogen bonds, because of the steric hindrance of the three tert-butyl groups surrounding the $\mathrm{OH}$ donor group (Majerz \& Natkaniec, 2006).

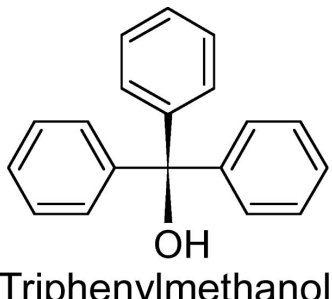

The case of triphenylmethanol, $\left(\mathrm{C}_{6} \mathrm{H}_{5}\right)_{3} \mathrm{COH}$, should be intermediate between tert-butanol and tri-tert-butylmethanol, since it can be used as a clathrate host for methanol (Weber et al., 1989), and may be hydrogen bonded to a water molecule (Batisai et al., 2016), dimethyl sulfoxide, dimethylformamide (Eckardt et al., 1999) or $\mathrm{Ph}_{3} \mathrm{P}=\mathrm{O}$ (Steiner, 2000). Indeed, unsolvated triphenylmethanol can be easily crystallized from

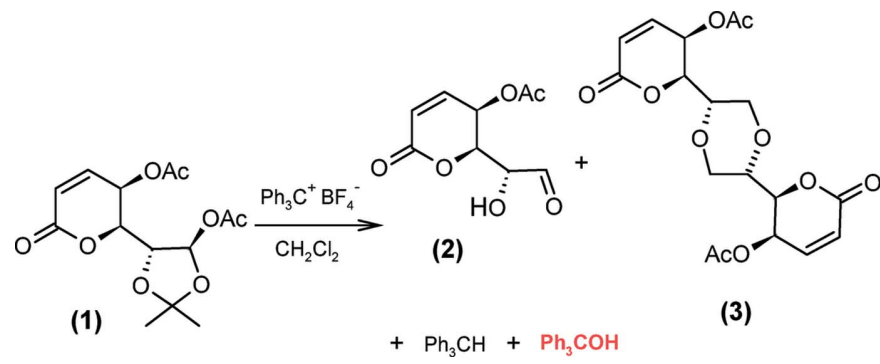

Figure 1

Synthetic step from which triphenylmethanol was crystallized. Note that product (2) was not obtained. benzene or ethanol, affording large well-shaped clear colourless single crystals. However, these crystals are always weakly diffracting samples, as a consequence of a severe structural disorder (vide infra). The resulting ratio of observed to measured reflections is then quite low, which, in turn, makes the refinement very difficult. First attempts to refine a reasonable model failed (Weber et al., 1989), and it was only in 1992 that the crystal structure was published (Ferguson et al., 1992), based on room-temperature intensities measured on a CAD-4 diffractometer, with Mo $K \alpha$ radiation. 2467 unique reflections were used, of which $41 \%$ were observed [ $I>$ $2.5 \sigma(I)]$, and the structure was refined with a structural motif described as a 'hydrogen-bonded pyramidal tetramer which is disordered (71/29) about two interpenetrating sites'. The refinement was of limited accuracy and converged to $R=0.083$ and $w R=0.068$ with rigid idealized phenyl rings for all molecules, and isotropic atoms in the minor-disordered part of the asymmetric unit (253 variable parameters).

Although the structure reported by Ferguson et al. was incomplete, since hydroxy $\mathrm{H}$ atoms could not be located, the savoir-faire used by this team is quite impressive. They were able to solve and refine this challenging disordered structure, while others probably gave up by arguing that crystals were badly twinned. Above all, they did not attempt to over-interpret their data, and were aware that hydroxy $\mathrm{H}$ atoms were very imprecisely determined in their X-ray diffraction experiment. However, they indirectly recognized and described the presence of a weak hydrogen-bonding scheme, reflected in intermolecular $\mathrm{O}$. . O contacts.

In 1999, Serrano-González et al. (1999) published a more elaborate article, focussed on the characterization of the hydrogen-bonding arrangement in triphenylmethanol, using neutron $(T=100 \mathrm{~K})$ and X-ray diffraction data $(T=113$ and 
$293 \mathrm{~K}$ ), as well as solid-state ${ }^{13} \mathrm{C}$ NMR spectroscopy. A reliable structure based on neutron diffraction data was obtained, showing that each independent $\mathrm{OH}$ group has the $\mathrm{H}$ atom disordered over three sites. This model was then a suitable starting point for the refinement of X-ray structures, both at 113 and $293 \mathrm{~K}$. Unfortunately, the final atomic coordinates were never deposited in the CSD, and there is no CIF available as supporting information. Fractional coordinates are tabulated, however, for $\mathrm{X}$-ray refinements, $\mathrm{H}$ atoms are missing. Moreover, even using the favourable neutron scattering length for the protium nucleus, it was not possible to complete the structure. As stated in this article 'The hydroxy hydrogens of the minor tetramer could not be located from the difference Fourier map, and these hydrogen atoms were inserted in calculated positions based on those determined [...] for the major tetramer'.

We have now completed these works, using X-ray data collected at room temperature and low temperature with the $\mathrm{Ag} K \alpha$ radiation, revealing the accurate localization of the hydroxy $\mathrm{H}$ atoms in the disordered structure. A comprehensive insight into the hydrogen-bonding scheme that held together the tetrameric clusters is now afforded.

\section{Experimental}

\subsection{Synthesis and crystallization}

We obtained triphenylmethanol as a by-product during the oxidative hydrolysis of the diacetylated compound (1), following a method proposed by Barton et al. (1972) (Fig. 1). Compound (1) (0.100 g, $0.318 \mathrm{mmol})$ and triphenylcarbenium tetrafluoroborate $(0.099 \mathrm{~g}, 0.380 \mathrm{mmol})$ were mixed in dry $\mathrm{CH}_{2} \mathrm{Cl}_{2}(20 \mathrm{ml})$ and stirred for $15 \mathrm{~min}$ at room temperature. A saturated solution of $\mathrm{NaHCO}_{3}$ was then added $(10 \mathrm{ml})$, the organic phase dried over $\mathrm{Na}_{2} \mathrm{SO}_{4}$ and the crude product chromatographed (silica gel, hexane-ethyl acetate, 90:10 v/v). The expected hydroxy aldehyde (2) was not observed, and the dimer (3) was isolated instead, mixed with triphenylmethane and triphenylmethanol. It was not possible to separate (3) and triphenylmethanol by chromatography, whereby the mixture was purified by crystallization in hexane-ethyl acetate (95:5 v/ $v$ ), affording large single crystals of triphenylmethanol [yield 30 mg; m.p. 431-433 K, literature 434-435 K (Zeiss \& Tsutsui, 1953)]. ${ }^{1} \mathrm{H}$ NMR ( $\left.\mathrm{CDCl}_{3} / \mathrm{TMS}, 300 \mathrm{MHz}\right): \delta 2.8(s, 1 \mathrm{H}, \mathrm{OH})$, $7.30(s, 15 \mathrm{H}, \mathrm{Ph}) \cdot{ }^{13} \mathrm{C}$ NMR $\left(\mathrm{CDCl}_{3} / \mathrm{TMS}, 75 \mathrm{MHz}\right): \delta 99.8$ $(\mathrm{C}-\mathrm{OH}), 127.2,127.9,146.8(\mathrm{Ph})$.

\subsection{Refinements}

Crystal data, data collection and structure refinement details are summarized in Table 1 for the two different crystals obtained from a single crystallization batch, but diffracted at different temperatures, i.e. 153 and $295 \mathrm{~K}$. The disorder in the asymmetric unit was solved using the low-temperature data set, and all phenyl rings were restrained to be flat, with standard deviations of $0.1 \AA^{3}$. Additionally, the phenyl rings in the minor part (molecules $C$ and $D$ ) were restrained to have 1,3 distances similar to those in the corresponding rings of the disordered counterpart (molecules $A$ and $B$ ), within standard deviations of $0.02 \AA$. $\mathrm{H}$ atoms in the phenyl rings were placed in idealized positions and refined as riding to their carrier $\mathrm{C}$ atoms, with $U_{\text {iso }}(\mathrm{H})=1.2 U_{\text {eq }}(\mathrm{C})$. Hydroxy $\mathrm{H}$ atoms were

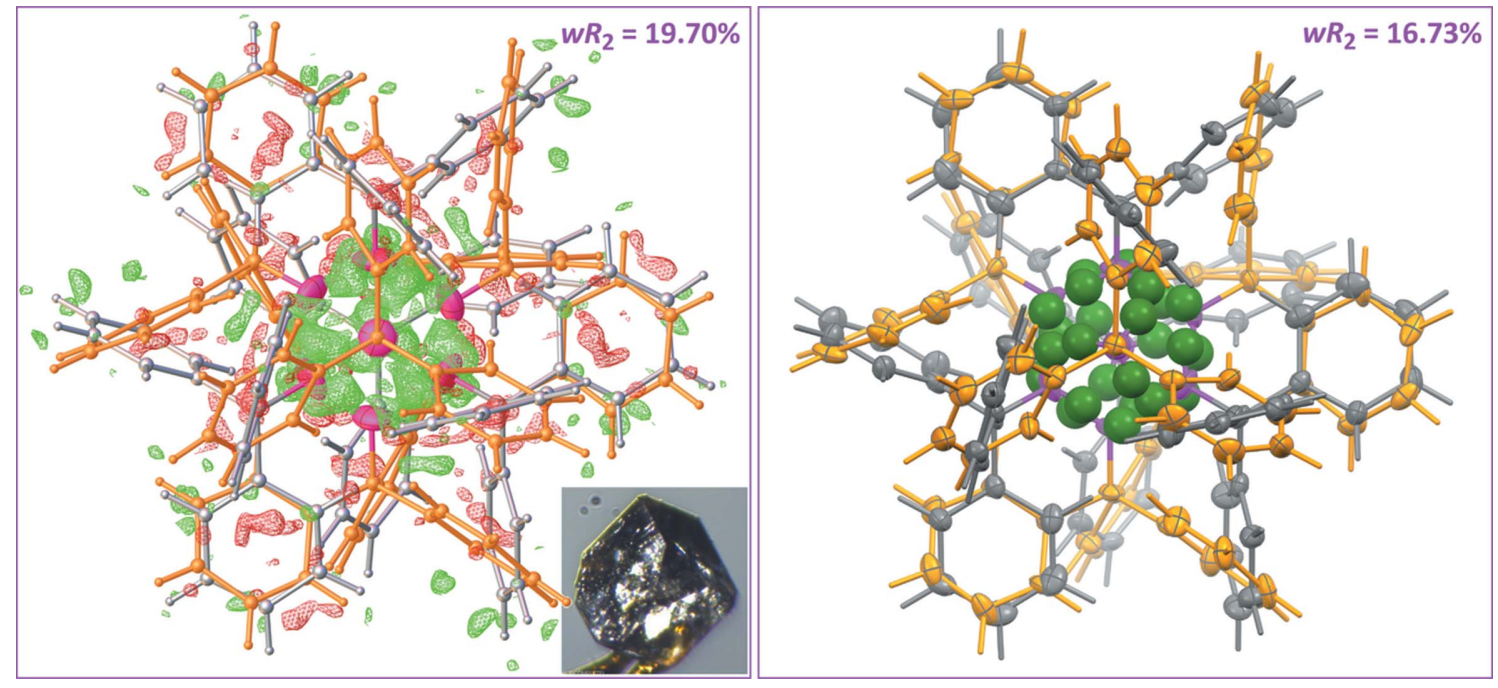

Figure 2

(Left) Difference electron-density map computed using low-temperature data, after refining the full disordered model but omitting hydroxy $\mathrm{H}$ atoms. Grey molecules correspond to the main part $A / B$ (occupancy 0.74) and gold molecules to the minor part $C / D$ (occupancy 0.26 ); hydroxy $\mathrm{O}$ atoms are represented with magenta ellipsoids. Two tetramers are displayed in a projection along the threefold crystallographic axis. The difference map is plotted at the 0.33 e $\AA^{-3}$ level (green wire for $\Delta \rho>0$ and red wire for $\Delta \rho<0$; Dolomanov et al., 2009). Note how most of the positive residuals are concentrated within the cavity delimited by the eight clustered $\mathrm{O}$ atoms. The inset is the crystal used for data collection. Note the triangular face on the top of the crystal, corresponding to the (003) face. (Right) Final model, including 24 disordered hydroxy H atoms, shown as green spheres with a radius corresponding to $33 \%$ of the van der Waals radius. All C and O atoms are displayed with displacement ellipsoids at the $20 \%$ probability level (Mercury; Macrae et al., 2008). 
found in a difference map (Fig. 2, left) and refined with $U_{\text {iso }}(\mathrm{H})=1.5 U_{\text {eq }}(\mathrm{O})$. Atoms $\mathrm{H} 1 A$ and $\mathrm{H} 1 C$ are disordered by symmetry, and their site occupancies were fixed as one-third of the occupancy of the part to which they belong. The hydroxy $\mathrm{H}$ atoms for molecules in general positions are disordered over sites $\mathrm{H} 1 B A, \mathrm{H} 1 B B$ and $\mathrm{H} 1 B C$ for molecule $B$, and $\mathrm{H} 1 D A, \mathrm{H} 1 D B$ and $\mathrm{H} 1 D C$ for molecule $D$, and the occupancy for each site was also fixed as one-third of the occupancy of the part to which it belongs. The geometry of the $\mathrm{C}-\mathrm{O}-\mathrm{H}$ groups was first restrained to a sensible target, by restraining distances to $\mathrm{O}-\mathrm{H}=0.85$ (1) $\AA$ and $\mathrm{C} \cdots \mathrm{H}=1.93$ (2) $\AA$ in molecules $A$ and $C$; for molecules $B$ and $D$, the applied restraints were $\mathrm{O}-\mathrm{H}=d, \mathrm{H} \cdots \mathrm{H}=(8 / 3)^{1 / 2} \times d$ and $\mathrm{C} \cdots \mathrm{H}=$ $2.27 \times d$, where $d$ is a common free variable. Standard deviations for these restraints were $0.02,0.03$ and $0.03 \AA$, respectively. After convergence, the positions of all hydroxy $\mathrm{H}$ atoms were fixed, and these atoms were refined as riding on their carrier $\mathrm{O}$ atoms. The final model for the complete structure at $153 \mathrm{~K}$ was refined against data collected at $295 \mathrm{~K}$, with an extra restraint: in the minor-disordered part (molecules $C$ and $D$ ), rigid-bond restraints were applied with standard deviations of $0.004 \AA$ for the 1,2 and 1,3 distances (Thorn et al., 2012; Sheldrick, 2015b).

\section{Results and discussion}

The asymmetric unit of the trigonal cell includes two disordered parts with site-occupancy factors converging at $153 \mathrm{~K}$
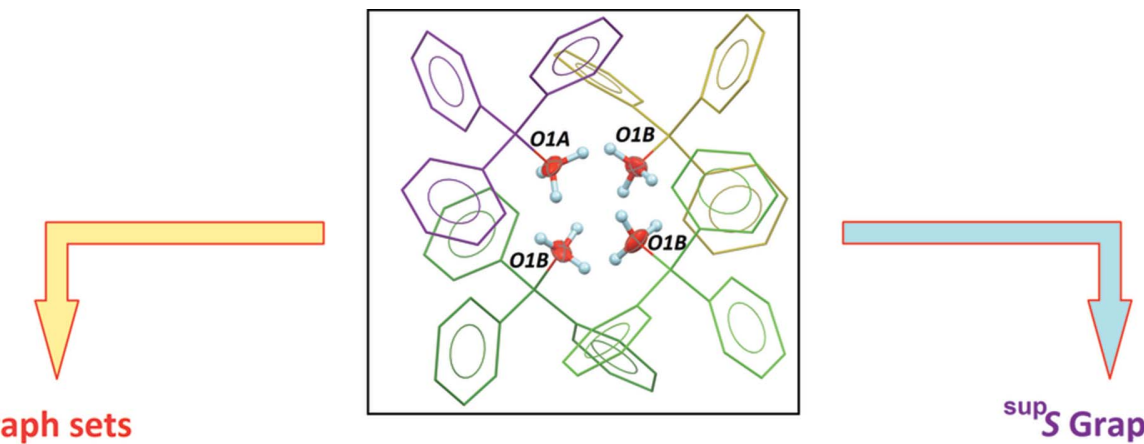

\section{${ }^{\sup } R$ Graph sets}
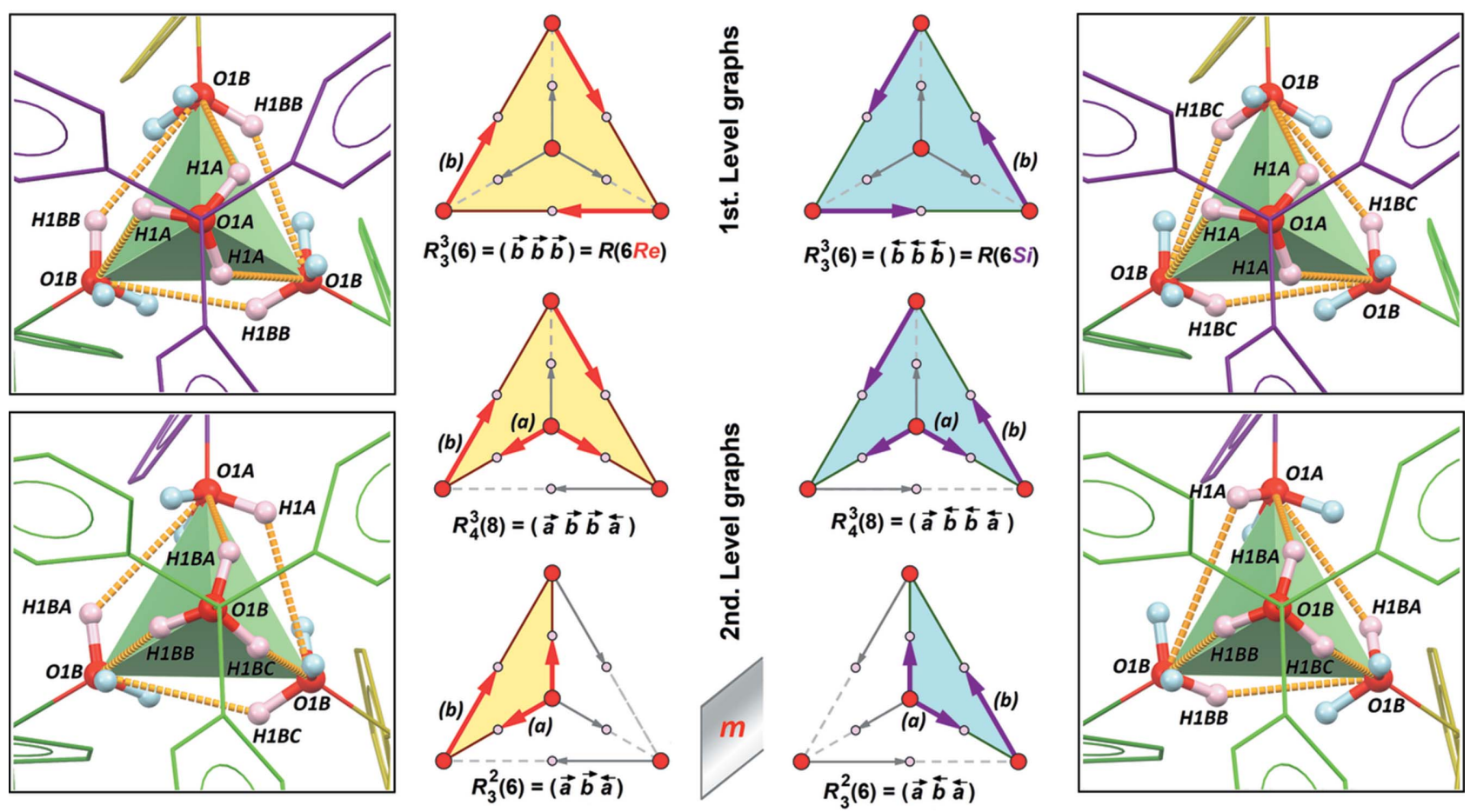

Figure 3

Hydrogen-bonding schemes in the tetramer formed by $A / B$ molecules. The top figure shows the arrangement of the four molecules and the 12 hydroxy $\mathrm{H}$-atom sites. The left and right panels represent right- $\left({ }^{\sup } R\right)$ and left-handed $\left({ }^{\text {sup }} S\right)$ supramolecular clusters, respectively. The first figure is oriented down the crystallographic threefold axis and the other is oriented down a noncrystallographic threefold axis. Each cluster comprises six hydrogen bonds (dashed gold bonds), involving six $\mathrm{H}$-atom sites (pink $\mathrm{H}$ atoms). Topological graphs $G(4,6)$ for supramolecular clusters based on $\mathrm{O}-\mathrm{H} \cdots \mathrm{O}$ hydrogen bonds are represented in the centre. Nodes are represented as red balls ( $\mathrm{O}$ atoms). Arrows forming a ring $R_{d}^{a}(n)$ are stacked over $\mathrm{O}-\mathrm{H}$ covalent bonds and oriented in the direction $d \rightarrow a$, where $d$ is the donor and $a$ the acceptor for a hydrogen bond. Arrows involved in a ring are shown in bold, while those not participating in a ring are greyed out. Polygons delimited by $R$ rings in the 2 -space are coloured yellow and blue for ${ }^{\text {sup }} R$ and ${ }^{\text {sup }} S$ clusters, respectively, and rings in the projection plane are read clockwise in all cases. For the first-level graphs, Re stands for Rectus and Si for Sinister. Note that all figures on the left are mirror images of the figures on the right, including descriptors of the $R$ rings. 
Table 2

Hydrogen-bond geometry $\left(\AA{ }^{\circ}\right)$ for the $153 \mathrm{~K}$ data.

\begin{tabular}{lllll}
\hline$D-\mathrm{H} \cdots A$ & $D-\mathrm{H}$ & $\mathrm{H} \cdots A$ & $D \cdots A$ & $D-\mathrm{H} \cdots A$ \\
\hline $\mathrm{O} 1 A-\mathrm{H} 1 A \cdots \mathrm{O} 1 B$ & 0.85 & 2.33 & $2.869(3)$ & 121 \\
$\mathrm{O} 1 B-\mathrm{H} 1 B A \cdots \mathrm{O} 1 A$ & 0.84 & 2.27 & $2.869(3)$ & 129 \\
$\mathrm{O} 1 B-\mathrm{H} 1 B B \cdots \mathrm{O} 1 B^{\mathrm{i}}$ & 0.82 & 2.21 & $2.869(3)$ & 138 \\
$\mathrm{O} 1 B-\mathrm{H} 1 B C \cdots \mathrm{O} 1 B^{\mathrm{ii}}$ & 0.86 & 2.24 & $2.869(3)$ & 130 \\
$\mathrm{O} 1 C-\mathrm{H} 1 C \cdots \mathrm{O} 1 D$ & 0.85 & 2.22 & $2.856(7)$ & 131 \\
$\mathrm{O} 1 D-\mathrm{H} 1 D A \cdots \mathrm{O} 1 C$ & 0.84 & 2.24 & $2.856(7)$ & 131 \\
$\mathrm{O} 1 D-\mathrm{H} 1 D B \cdots \mathrm{O} 1 D^{\mathrm{ii}}$ & 0.82 & 2.38 & $2.951(8)$ & 127 \\
$\mathrm{O} 1 D-\mathrm{H} 1 D C \cdots \mathrm{O} 1 D^{\mathrm{i}}$ & 0.85 & 2.33 & $2.951(8)$ & 131 \\
\hline
\end{tabular}

Symmetry codes: (i) $-y+1, x-y, z$; (ii) $-x+y+1,-x+1, z$.

towards 0.7436 (17) (molecules $A$ and $B$ hereafter) and 0.2564 (17) (molecules $C$ and $D$ hereafter), close to the occupancies reported by Ferguson et al. of 0.71 and 0.29. Each part contains two independent molecules, one of which has the $\sigma \mathrm{C}-\mathrm{O}$ bond lying on the threefold axis in the space group $R \overline{3}$, while the other is located in a general position. The arrangement of these four disordered molecules generates overlapped phenyl rings in the asymmetric unit, making the refinement of displacement parameters a tedious task (see \$2.2). However, the molecular structure based on data collected at $153 \mathrm{~K}$ can be considered as satisfactory, although the refinement was carried out with restrained geometry for the phenyl rings. The refinement based on data collected at $295 \mathrm{~K}$ is not as easy, since the scattering power of the crystal decreases dramatically: the fraction of observed data $[I / \sigma(I)>2]$ drops from $50 \%$ at $153 \mathrm{~K}$ to $36 \%$ at $295 \mathrm{~K}$. However, the structure is essentially unmodified, and occupancies for the disordered parts refined to 0.761 (3) and 0.239 (3). At both temperatures, all non-H atoms could be refined anisotropically (see Fig. 2, right), after which phenyl $\mathrm{H}$ atoms were placed in idealized positions.

The localization of the hydroxy $\mathrm{H}$ atoms was far more complex. A difference map using room-temperature data is useless, in contrast to the map computed with data collected at low temperature. At $153 \mathrm{~K}$, most of the positive residuals are found close to the $\mathrm{O}$ atoms (Fig. 2, left), allowing the determination of sensible coordinates for $\mathrm{H}$ atoms disordered by symmetry ( $\mathrm{H} 1 A$ and $\mathrm{H} 1 C$ for molecules $A$ and $C$ in special positions). At this point, the highest residuals are found close to $\mathrm{O} 1 B$, forming a tetrahedral geometry with $\mathrm{O} 1 B$; although molecule $B$ is located in a general position, the $\mathrm{O}-\mathrm{H}$ group emulates the disorder imposed by symmetry in molecule $A$ (see top inset in Fig. 3). The same situation is repeated for molecule $D$, with much smaller residuals because this molecule belongs to the minor part of the disordered asymmetric unit. Ultimately, all the molecules in the crystal have their hydroxy $\mathrm{H}$ atoms disordered over three sites, and once the asymmetric unit is expanded to tetramers, eight independent molecules are clustered in such a way that the cavity delimited by eight $\mathrm{O}$ atoms is filled with 24 sites for disordered hydroxy $\mathrm{H}$ atoms (Fig. 2, right). Each $\mathrm{C}-\mathrm{O}-\mathrm{H}$ group can also be seen as a rigid group rotating about its $\mathrm{C}-\mathrm{O}$ axis, producing for the $\mathrm{H}$ atom an electron density smeared out over a ring; nevertheless, the free rotation should be hindered through the formation of weak hydrogen bonds (Schröder et al., 2004). Such a description would be consistent with ${ }^{2} \mathrm{H}$ NMR spectroscopy experiments carried out on $\mathrm{Ph}_{3} \mathrm{COD}$, showing that each hydroxy group is dynamic by rotation about the $\mathrm{C}-\mathrm{OD}$ bond, on the $10^{-3}$ to $10^{-8}$ s time scale (Aliev et al., 1998).

All $\mathrm{OH}$ groups behave as donor groups for intermolecular $\mathrm{O}-\mathrm{H} \cdots \mathrm{O}$ hydrogen bonds. For the main part $A / B$, with occupancy $=0.74$, three $B$ molecules placed close to the threefold axis are connected to form an $R_{3}^{3}(6)$ ring, corresponding to a first-level graph with $\mathrm{H} 1 B B$ as donor (Table 2, entry 3 ). This motif is repeated with $\mathrm{H} 1 B C$ (Table 2, entry 4), however, if the crystal orientation is preserved, this ring motif is enantiomorphic with the previous one. Finally, the third disordered site for the hydroxy $\mathrm{H}$ atom, $\mathrm{H} 1 B A$, is engaged in a second-level graph with $\mathrm{O} 1 A$ as acceptor (Table 2, entry 2), giving a ring motif $R_{3}^{2}(6)$. Site O1 $A$ also serves as a donor, forming three symmetry-equivalent $\mathrm{O} 1 A-\mathrm{H} 1 A \cdots \mathrm{O} 1 B$ hydro-
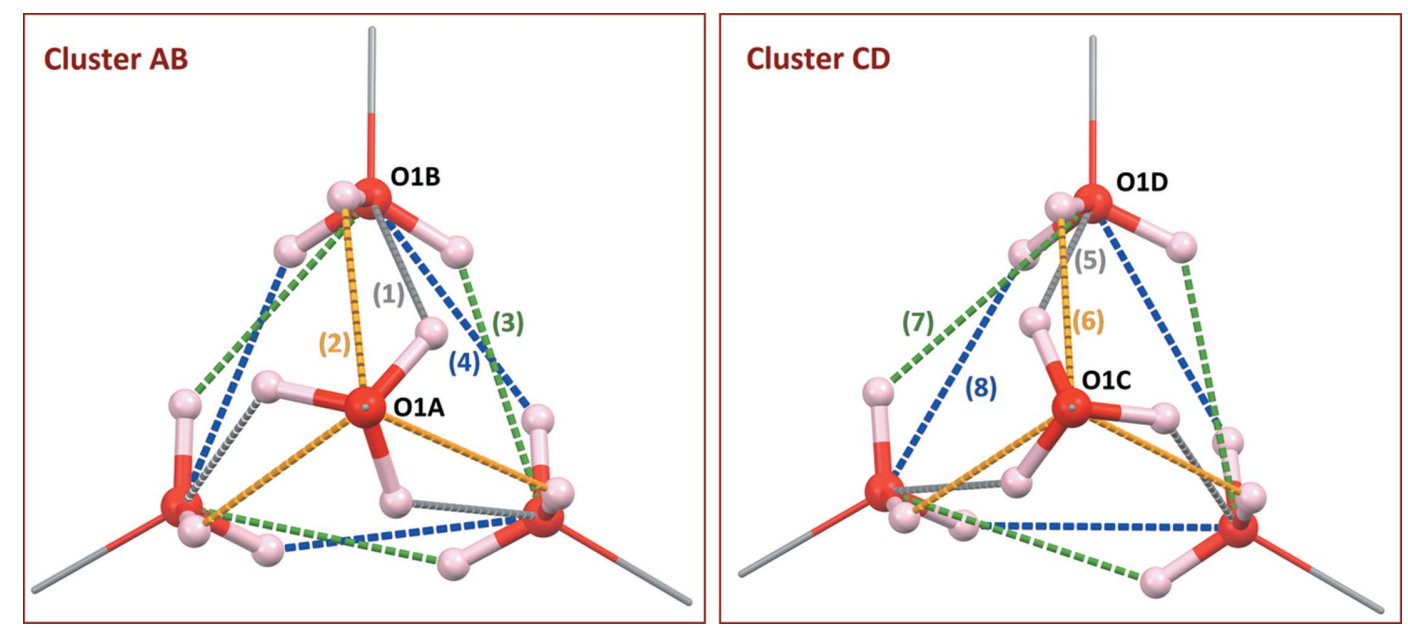

Figure 4

Complete set of hydrogen bonds, represented as dashed lines, in the tetramer formed by molecules $A$ and $B$ (left), and in the tetramer formed by molecules $C$ and $D$ (right). Figures are oriented down the crystallographic threefold axis. Labels (1) . .(8) on hydrogen bonds indicate the entry in Table 2. Each cluster has four independent bonds, affording 12 bonds for the tetramer, consistent with the $C_{3}$ point symmetry of the tetramer. These figures can be compared to the model published in 1999 (see Fig. 4 in Serrano-González et al., 1999). 
Table 3

Hydrogen-bond geometry $\left(\AA{ }^{\circ}\right)$ for the $295 \mathrm{~K}$ data.

\begin{tabular}{lllll}
\hline$D-\mathrm{H} \cdots A$ & $D-\mathrm{H}$ & $\mathrm{H} \cdots A$ & $D \cdots A$ & $D-\mathrm{H} \cdots A$ \\
\hline $\mathrm{O} 1 A-\mathrm{H} 1 A \cdots \mathrm{O} 1 B$ & 0.85 & 2.56 & $2.917(3)$ & 106 \\
$\mathrm{O} 1 B-\mathrm{H} 1 B A \cdots \mathrm{O} 1 A$ & 0.82 & 2.27 & $2.917(3)$ & 136 \\
$\mathrm{O} 1 B-\mathrm{H} 1 B B \cdots \mathrm{O} 1 B^{\mathrm{i}}$ & 0.82 & 2.31 & $2.912(3)$ & 131 \\
$\mathrm{O} 1 B-\mathrm{H} 1 B C \cdots \mathrm{O} 1 B^{\mathrm{ii}}$ & 0.85 & 2.31 & $2.912(4)$ & 128 \\
$\mathrm{O} 1 C-\mathrm{H} 1 C \cdots \mathrm{O} 1 D$ & 0.85 & 2.27 & $2.916(13)$ & 133 \\
$\mathrm{O} 1 D-\mathrm{H} 1 D A \cdots \mathrm{O} 1 C$ & 0.83 & 2.26 & $2.916(13)$ & 136 \\
$\mathrm{O} 1 D-\mathrm{H} 1 D B \cdots \mathrm{O} 1 D^{\mathrm{ii}}$ & 0.83 & 2.35 & $2.947(13)$ & 130 \\
$\mathrm{O} 1 D-\mathrm{H} 1 D C \cdots \mathrm{O} 1 D^{\mathrm{i}}$ & 0.84 & 2.33 & $2.947(13)$ & 132 \\
\hline
\end{tabular}

Symmetry codes: (i) $-y+1, x-y, z$; (ii) $-x+y+1,-x+1, z$.

gen bonds (Table 2, entry 1), and is involved in the largest rings, $R_{4}^{3}(8)$. All rings are depicted in Fig. 3, along with schematic representations of the corresponding graphs and their pathways [i.e. the constructor graphs and the qualitative descriptors, in the terminology coined by Motherwell et al. (1999)].

The tetramer based on $A$ and $B$ molecules includes 12 hydrogen bonds. Each disordered hydroxy $\mathrm{H}$ atom is engaged in a single hydrogen bond, and each $\mathrm{O}$ atom serves three times as acceptor (Fig. 4, left). The hydrogen-bonded supramolecular cluster formed in the tetramer is associated with a topological graph embedded in 3-space, i.e. $G(4,6)=\left[R_{3}^{3}(6)\right.$ $R_{4}^{3}(8) R_{3}^{2}(6)$ ], for which the faces are the $R(n)$ rings described in Fig. 3. In the parlance of graph theory, the finite directed graph $G(4,6)$ based on the 'pyramidal tetramer' mentioned by Ferguson et al. is regular, complete and intrinsically chiral (Flapan, 1995). The four nodes for $G(4,6)$ are provided by four molecules (or four hydroxy $\mathrm{O}$ atoms for simplicity) and the six arrows are oriented $\mathrm{O}-\mathrm{H} \cdots \mathrm{O}$ hydrogen bonds, the tail of the arrow being the donor $\mathrm{OH}$ group and the head being the acceptor $\mathrm{O}$ atom. It is noteworthy that for each right-handed $R(n)$ ring, there is one related left-handed ring, as illustrated in Fig. 3. For example, in the first-level $R_{3}^{3}(6)$ subgraph embedded in the 2-space, arrows rotate clockwise around the crystallographic threefold axis for the ring including arrows $\mathrm{O} 1 B-$ $\mathrm{H} 1 B B \cdots \mathrm{O} 1 B$ (Rectus face for topological graph $G$ ), and anticlockwise for the ring including arrows $\mathrm{O} 1 B-$ $\mathrm{H} 1 B C \cdots \mathrm{O} 1 B$ (Sinister face for topological graph $G$ ).

A topologically isomorphous graph $G^{\prime}(4,6)$ can be built with the minor part of the asymmetric unit, including hydrogen bonds similar to those described for the main tetramer, although the relative positions of the $12 \mathrm{H}$-atom sites is modified through a small rotation around the $\mathrm{C} 1 \mathrm{C}-$ $\mathrm{O} 1 \mathrm{C}$ axis (Fig. 4, right). Therefore, the mixture of eight molecules built on the asymmetric unit, as represented in Fig. 2, affords a racemic mixture of supramolecular enantiomorphic tetramers ${ }^{\text {sup }} R$ and ${ }^{\text {sup }} S$ built on 24 hydrogen bonds. Obviously, the molecules themselves are achiral, but the supramolecular chirality results from the asymmetric configuration of the hydrogen bonds (Sasaki et al., 2014). Neither the unit cell nor the crystal are chiral objects, since the molecule crystallizes in a centrosymmetric space group, $R \overline{3}$.

The set of 24 hydrogen bonds depicted in Fig. 4 comprises only weak hydrogen bonds, with $\mathrm{H}$... O separations in the range 2.21-2.38 $\AA$ and $\mathrm{O}-\mathrm{H}$. . O angles far from linearity, in the range $121.4-138.2^{\circ}$, at $153 \mathrm{~K}$. The refinement using roomtemperature data indicates that the supramolecular chiral clusters are retained, although hydrogen bonds are slightly weakened by $c a 0.06 \AA$ for $\mathrm{H}$. . O separations (compare Tables 2 and 3 ). These geometric parameters were compared with those found in other supramolecular networks formed in the crystalline state by tertiary alcohols, using the methodology developed at the CCDC (Wood et al., 2009). Parameters for intermolecular $\mathrm{O}-\mathrm{H} \cdots \mathrm{O}$ contacts in tertiary alcohols were retrieved from the current release of the CSD, omitting disordered, polymeric and ionic structures. Hydroxy $\mathrm{H}$-atom positions were normalized within ConQuest to $\mathrm{O}-\mathrm{H}$ $=0.993 \AA$, in order to avoid systematic errors in contact distances (Bruno et al., 2002). The search was limited to organic compounds not flagged with errors, and reported with $R_{1}<0.075$, affording 1215 hits, corresponding to 1812 raw data $(d, \theta)$, where $d$ is the $\mathrm{H} \cdots \mathrm{O}$ distance and $\theta$ is the $\mathrm{O}-\mathrm{H} \cdots \mathrm{O}$ angle. Only contacts with $d$ shorter than the van der Waals (vdW) distance were retained $\left[r_{\mathrm{vdW}}(\mathrm{H})+r_{\mathrm{vdW}}(\mathrm{O})=2.72 \AA\right.$; Bondi, 1964]. Data were converted into spherical polar coordinates $(x, y)$, where $x=(d / 2.72)^{3}$ and $y=1-\cos (180-\theta)$,

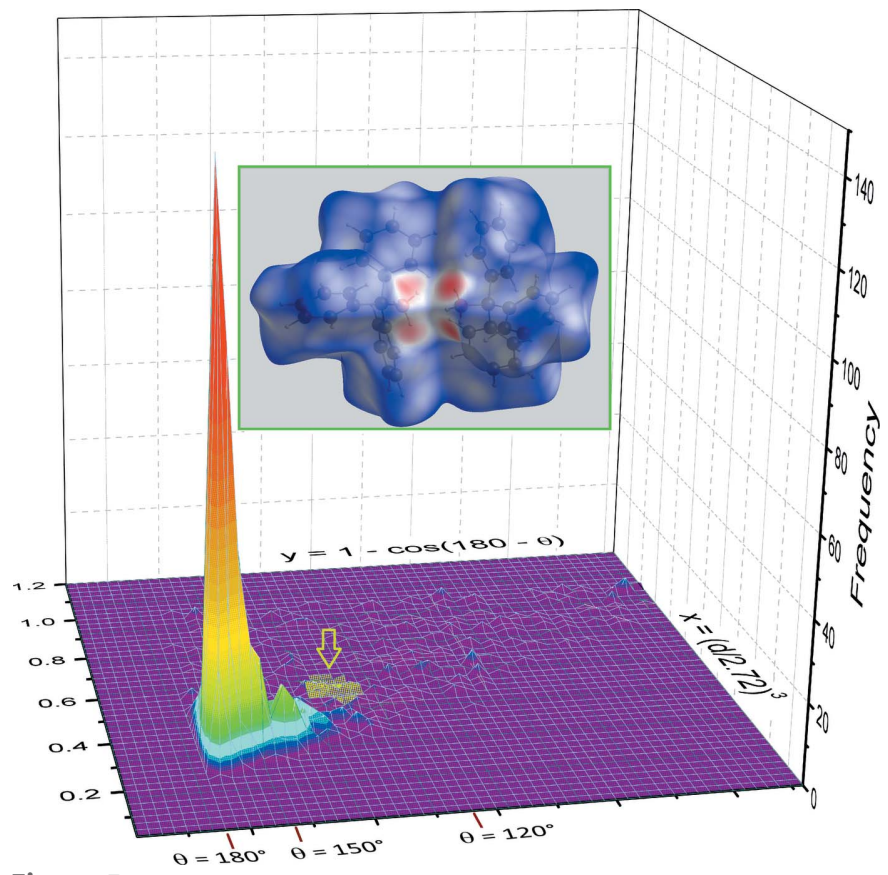

Figure 5

Histogram of the $\mathrm{O}-\mathrm{H} \cdots \mathrm{O}$ intermolecular hydrogen-bond geometry in the crystal structures of tertiary alcohols, in spherical polar coordinates $(x, y)$, with the CSD frequency shown in the third dimension (OriginLab, 2012). A $\log _{10}$ rainbow colour scheme is used to highlight small frequencies. Some values for the $\mathrm{O}-\mathrm{H} \cdots \mathrm{O}$ angles $\theta$ are reported on axis $y$, for reference. Note the similarity of the distribution with that depicted in the CCDC article about the directionality of hydrogen bonds (Wood et al., 2009; see Fig. 2 in this article). The green patch marked with an arrow in the $(x, y)$ plane defines the area for hydrogen bonds in the $A / B$ tetrameric cluster of the title compound at $153 \mathrm{~K}$. The inset shows the Hirshfeld surface mapped over $d$ ( -1 to $1 \AA$ ) for the $A / B$ molecules at $153 \mathrm{~K}$ (Turner et al., 2017); each of the four red patches on the surface is related to a single node for the topological graph $G(4,6)$ of the $A / B$ tetramer (see Fig. 3). 
Table 4

Comparison between the three X-ray structures of triphenylmethanol determined at room temperature.

\begin{tabular}{llll}
\hline Date of publication & $1992^{a}$ & $1999^{b}$ & $2019^{c}$ \\
Diffractometer & CAD-4 & R-Axis II & Stadivari $^{d}$ \\
Detector & NaI scintillator & Image plate & HPAD $^{d}$ \\
$T(\mathrm{~K})$ & 294 & 293 & 295 \\
No. independent reflections & 2467 & 3448 & 4523 \\
Refined parameters & 253 & 322 & 483 \\
Data resolution $(\AA)$ & 0.89 & 0.82 & 0.77 \\
Range for $\sigma(\mathrm{C}-\mathrm{C})$ & $0.040-0.007 \AA$ & - & $0.020-0.003 \AA$
\end{tabular}

Notes and references: (a) Ferguson et al. (1992); (b) Serrano-González et al. (1999); (c) this work; (d) Hybrid Pixel Array Detector.

assuming that $d$ and $\theta$ are expressed in $\AA$ and ${ }^{\circ}$, respectively (Lommerse et al., 1996). A two-dimensional (2D) frequency binning histogram representation of the $(x, y)$ distribution shows a sharp peak about $(d, \theta)=\left(1.82 \AA, 180^{\circ}\right)$, as expected for $\mathrm{O}-\mathrm{H} \cdots \mathrm{O}$ hydrogen bonds (Fig. 5). A significant frequency is still observed about $(d, \theta)=\left(1.87 \AA, 145^{\circ}\right)$. Outside these well-defined territories, the observed frequency collapses.

Interestingly, the title compound displays $\mathrm{O}-\mathrm{H} \cdots \mathrm{O}$ contacts on the borderline between truly hydrogen-bonded alcohols and the near-zero frequency area (see green patch on the ground level in Fig. 5). However, although of very limited strength, hydrogen bonds in the tetramers depicted in Fig. 3 are genuinely present, as reflected in the Hirshfeld surface for any pair of molecules involved in a tetrameric supramolecular cluster (Fig. 5, inset). In other words, triphenylmethanol could represent the boundary between hydrogen-bonded and nonhydrogen-bonded tertiary alcohols. This also opens the possibility that a phase transition occurs for triphenylmethanol somewhere between $T=293$ and $433 \mathrm{~K}$ (melting point), if thermal energy $k T$ is able to dismantle the network of weak hydrogen bonds.

In order to evaluate the stability of the noncovalent bonds in this crystal, George Ferguson and co-workers came across a more chemical strategy, by determining the crystal structures of molecules isoelectronic with triphenylmethanol (Glidewell \& Ferguson, 1994). Their hypothesis was that 'with only modest changes in the steric demands at the unique central $\mathrm{C}$ atom, while keeping the number of hydrogen-bond donors and acceptors unchanged, the patterns of hydrogen bonding can be altered drastically'. Indeed, diphenyl(pyridin-4-yl)methanol has a simple achiral supramolecular structure based on $C$ (7) chains. For triphenylmethanamine, two polymorphic forms have been described: the orthorhombic phase does not form hydrogen bonds at all (Glidewell \& Ferguson, 1994), while the triclinic phase features dimers through the formation of $\mathrm{N}-\mathrm{H} \cdots \mathrm{N}$ hydrogen bonds, due to the statistical disordering of the amino $\mathrm{H}$ atoms (Khrustalev et al., 2009; Schulz et al., 2013). The $\mathrm{NH}_{2}$ group then displays a geometry reminiscent of that of the $\mathrm{OH}$ groups in triphenylmethanol. However, no chiral supramolecular clusters are formed with the amine, and the asymmetric unit includes a single nondisordered molecule. A rhombohedral polymorph for this amine has also been deposited recently; unfortunately, after inspection of this structure, it turns out that the formula is wrong: the diffracted crystal was almost certainly triphenylmethanol (Bagchi et al., 2014; $R \overline{3}$ space group, $T=298 \mathrm{~K}, R_{1}=10 \%$ ). In the opposite direction, strong $\mathrm{O}-\mathrm{H} \cdots \mathrm{O}$ hydrogen bonds can be restored in sterically hindered tertiary alcohols by just adding a methylene group: in the crystal structure of triphenylethanol, $\mathrm{Ph}_{3} \mathrm{CCH}_{2} \mathrm{OH}$ (nondisordered $P 2_{1} / c$ crystal, $Z^{\prime}=2$; Ferguson et al., 1994), molecules aggregate into discrete achiral $R_{4}^{4}(8)$ rings. In comparison with triphenylmethanol, the prochiral character of the supramolecular structure is lost, and the compound lies within a sharp peak of 'normal' crystal structures in a $(x, y)$ distribution similar to that depicted for tertiary alcohols in Fig. 5.

\section{Concluding remarks}

Triphenylmethanol is a small simple molecule with a structure unexpectedly difficult to refine compared, for example, to that of triphenylsilanol (Bowes et al., 2002). It is worthwhile to consider the evolution of X-ray diffractometry over the last 25 years, using triphenylmethanol as a benchmark (Table 4; data at room temperature were retained in order to avoid biases). The time taken for data collection is almost identical in the three cases, ca $20-30 \mathrm{~h}$, and there is little doubt that the diffracted samples were of similar quality. Over time, a steady progress is noted. Development of new technologies for the detection of scattered X-ray photons seems to be the key point, in such a way that location of tiny fractions of electrons in the crystal space is now routinely affordable, outside the multipole density formalism. There is indeed a consensus that the hybrid pixel detectors with CdTe or GaAs sensors represent the ultimate state-of-art technology in this field, since they detect all scattered X-rays, with 100\% efficiency and without any noise (Allé et al., 2016). For the herein presented study, a Pilatus detector was used, with a $1000 \mu \mathrm{m}$-thick silicon sensor, which has a quantum efficiency of $50 \%$ for $22.2 \mathrm{keV}$ photons (Ag $K \alpha$ radiation).

\section{Acknowledgements}

The submitting author thanks an anonymous referee for his guidance regarding the accurate interpretation of the difference map depicted in Fig. 2.

\section{Funding information}

Funding for this research was provided by: Consejo Nacional de Ciencia y Tecnología (grant No. 268178; scholarship No. 304678); VIEP-BUAP (grant No. 100317000-VIEP2018).

\section{References}

Aliev, A. E., MacLean, E. J., Harris, K. D. M., Kariuki, B. M. \& Glidewell, C. (1998). J. Phys. Chem. B, 102, 2165-2175.

Allé, P., Wenger, E., Dahaoui, S., Schaniel, D. \& Lecomte, C. (2016). Phys. Scr. 91, 063001.

Bagchi, V., Paraskevopoulou, P., Das, P., Chi, L., Wang, Q., Choudhury, A., Mathieson, J. S., Cronin, L., Pardue, D. B., Cundari, T. R., Mitrikas, G., Sanakis, Y. \& Stavropoulos, P. (2014). J. Am. Chem. Soc. 136, 11362-11381. 
Barton, D. H. R., Magnus, P. D., Smith, G., Streckert, G. \& Zurr, D. (1972). J. Chem. Soc. Perkin Trans. 1, pp. 542-552.

Batisai, E., Su, H. \& Nassimbeni, L. R. (2016). CrystEngComm, 18, 5952-5958.

Batsanov, A. S. (2018). Acta Cryst. E74, 570-574.

Bondi, A. (1964). J. Phys. Chem. 68, 441-451.

Bowes, K. F., Glidewell, C. \& Low, J. N. (2002). Acta Cryst. C58, o409o415.

Bruno, I. J., Cole, J. C., Edgington, P. R., Kessler, M., Macrae, C. F., McCabe, P., Pearson, J. \& Taylor, R. (2002). Acta Cryst. B58, 389397.

Dobrzycki, L. (2018). Z. Kristallogr. Cryst. Mater. 233, 41-49.

Dolomanov, O. V., Bourhis, L. J., Gildea, R. J., Howard, J. A. K. \& Puschmann, H. (2009). J. Appl. Cryst. 42, 339-341.

Eckardt, K., Paulus, H., Fuess, H., Onoda-Yamamuro, N., Ikeda, R. \& Weiss, A. (1999). J. Inclusion Phenom. Macrocycl. Chem. 35, 431449.

Ferguson, G., Gallagher, J. F., Glidewell, C., Low, J. N. \& Scrimgeour, S. N. (1992). Acta Cryst. C48, 1272-1275.

Ferguson, G., Glidewell, C. \& Zakaria, C. M. (1994). Acta Cryst. C50, 928-931.

Flapan, E. (1995). J. Mol. Struct. (Theochem), 336, 157-164.

Glidewell, C. \& Ferguson, G. (1994). Acta Cryst. C50, 924-928.

Groom, C. R., Bruno, I. J., Lightfoot, M. P. \& Ward, S. C. (2016). Acta Cryst. B72, 171-179.

Khrustalev, V. N., Borisova, I. V., Zemlyansky, N. N. \& Antipin, M. Y. (2009). Acta Cryst. C65, o31-o34.

Lommerse, J. P. M., Stone, A. J., Taylor, R. \& Allen, F. H. (1996). J. Am. Chem. Soc. 118, 3108-3116.

Macrae, C. F., Bruno, I. J., Chisholm, J. A., Edgington, P. R., McCabe, P., Pidcock, E., Rodriguez-Monge, L., Taylor, R., van de Streek, J. \& Wood, P. A. (2008). J. Appl. Cryst. 41, 466-470.

Majerz, I. \& Natkaniec, I. (2006). J. Mol. Struct. 788, 93-101.
Malarski, Z. (1974). Mol. Cryst. Liq. Cryst. 25, 259-272.

Mootz, D. \& Stäben, D. (1993). Z. Naturforsch. Teil B, 48, 1325-1330.

Motherwell, W. D. S., Shields, G. P. \& Allen, F. H. (1999). Acta Cryst. B55, 1044-1056.

OriginLab (2012). OriginPro 9.1. OriginLab Corporation, Northampton, MA, USA.

Sasaki, T., Ida, Y., Isaki, I., Yuge, T., Uchida, Y., Tohnai, N. \& Miyata, M. (2014). Chem. Eur. J. 20, 2478-2487.

Schröder, L., Watkin, D. J., Cousson, A., Cooper, R. I. \& Paulus, W. (2004). J. Appl. Cryst. 37, 545-550.

Schulz, A., Villinger, A. \& Westenkirchner, A. (2013). Inorg. Chem. 52, 11457-11468.

Serrano-González, H., Harris, K. D. M., Wilson, C. C., Aliev, A. E., Kitchin, S. J., Kariuki, B. M., Bach-Vergés, M., Glidewell, C., MacLean, E. J. \& Kagunya, W. W. (1999). J. Phys. Chem. B, 103, 6215-6223.

Sheldrick, G. M. (2008). Acta Cryst. A64, 112-122.

Sheldrick, G. M. (2015a). Acta Cryst. A71, 3-8.

Sheldrick, G. M. (2015b). Acta Cryst. C71, 3-8.

Steiner, T. (2000). Acta Cryst. C56, 1033-1034.

Stoe \& Cie (2018). $X$-AREA and X-RED32. Stoe \& Cie, Darmstadt, Germany.

Thorn, A., Dittrich, B. \& Sheldrick, G. M. (2012). Acta Cryst. A68, 448-451.

Turner, M. J., McKinnon, J. J., Wolff, S. K., Grimwood, D. J., Spackman, P. R., Jayatilaka, D. \& Spackman, M. A. (2017). CrystalExplorer17. University of Western Australia.

Weber, E., Skobridis, K. \& Goldberg, I. (1989). J. Chem. Soc. Chem. Commun. pp. 1195-1197.

Westrip, S. P. (2010). J. Appl. Cryst. 43, 920-925.

Wood, P. A., Allen, F. H. \& Pidcock, E. (2009). CrystEngComm, 11, $1563-1571$

Zeiss, H. H. \& Tsutsui, M. (1953). J. Am. Chem. Soc. 75, 897-900. 


\section{supporting information}

Acta Cryst. (2019). C75, 1266-1273 [https://doi.org/10.1107/S2053229619010714]

Deciphering the hydrogen-bonding scheme in the crystal structure of triphenylmethanol: a tribute to George Ferguson and co-workers

Tomasa Rodríguez Tzompantzi, Aldo Guillermo Amaro Hernández, Rosa Luisa Meza-León and Sylvain Bernès

Computing details

For both structures, data collection: $X$-AREA (Stoe \& Cie, 2018); cell refinement: $X$-AREA (Stoe \& Cie, 2018); data reduction: $X$-AREA (Stoe \& Cie, 2018); program(s) used to solve structure: SHELXT2018 (Sheldrick, 2015a); program(s) used to refine structure: SHELXL2018 (Sheldrick, 2015b); molecular graphics: XP in SHELXTL-Plus (Sheldrick, 2008), Mercury (Macrae et al., 2008) and OLEX2 (Dolomanov et al., 2009); software used to prepare material for publication: publCIF (Westrip, 2010).

Triphenylmethanol (153K_data)

Crystal data

$\mathrm{C}_{19} \mathrm{H}_{16} \mathrm{O}$

$M_{r}=260.32$

Trigonal, $R \overline{3}: H$

$a=19.1399(5) \AA$

$c=26.7399(9) \AA$

$V=8483.4(5) \AA^{3}$

$Z=24$

$F(000)=3312$

$D_{\mathrm{x}}=1.223 \mathrm{Mg} \mathrm{m}^{-3}$

\section{Data collection}

Stoe Stadivari diffractometer

Radiation source: Sealed X-ray tube, Axo Astixf Microfocus source

Graded multilayer mirror monochromator

Detector resolution: 5.81 pixels $\mathrm{mm}^{-1}$

$\omega$ scans

Absorption correction: multi-scan

(X-AREA; Stoe \& Cie, 2018)

\section{Refinement}

Refinement on $F^{2}$

Least-squares matrix: full

$R\left[F^{2}>2 \sigma\left(F^{2}\right)\right]=0.052$

$w R\left(F^{2}\right)=0.167$

$S=1.00$

4399 reflections
Melting point: $433 \mathrm{~K}$

$\mathrm{Ag} K \alpha$ radiation, $\lambda=0.56083 \AA$

Cell parameters from 38242 reflections

$\theta=2.3-27.9^{\circ}$

$\mu=0.05 \mathrm{~mm}^{-1}$

$T=153 \mathrm{~K}$

Prism, colourless

$0.33 \times 0.29 \times 0.25 \mathrm{~mm}$

$T_{\min }=0.419, T_{\max }=1.000$

99452 measured reflections

4399 independent reflections

2222 reflections with $I>2 \sigma(I)$

$R_{\text {int }}=0.069$

$\theta_{\text {max }}=21.5^{\circ}, \theta_{\min }=2.3^{\circ}$

$h=-24 \rightarrow 24$

$k=-24 \rightarrow 24$

$l=-34 \rightarrow 34$

483 parameters

72 restraints

0 constraints

Primary atom site location: dual

Secondary atom site location: difference Fourier map 
Hydrogen site location: mixed $\mathrm{H}$-atom parameters constrained $w=1 /\left[\sigma^{2}\left(F_{\mathrm{o}}^{2}\right)+(0.0683 P)^{2}+2.9871 P\right]$ where $P=\left(F_{\mathrm{o}}^{2}+2 F_{\mathrm{c}}^{2}\right) / 3$
$(\Delta / \sigma)_{\max }<0.001$

$\Delta \rho_{\max }=0.18 \mathrm{e} \AA^{-3}$

$\Delta \rho_{\min }=-0.14$ e $\AA^{-3}$

Fractional atomic coordinates and isotropic or equivalent isotropic displacement parameters $\left(\hat{A}^{2}\right)$

\begin{tabular}{|c|c|c|c|c|c|}
\hline & $x$ & $y$ & $z$ & $U_{\text {iso }} * / U_{\text {eq }}$ & Occ. $(<1)$ \\
\hline O1A & 0.666667 & 0.333333 & $0.51277(9)$ & $0.0911(10)$ & $0.7436(17)$ \\
\hline $\mathrm{H} 1 \mathrm{~A}$ & 0.708695 & 0.373627 & 0.525137 & $0.137^{*}$ & $0.2479(6)$ \\
\hline $\mathrm{C} 1 \mathrm{~A}$ & 0.666667 & 0.333333 & $0.45915(12)$ & $0.0585(9)$ & $0.7436(17)$ \\
\hline $\mathrm{C} 2 \mathrm{~A}$ & $0.58184(15)$ & $0.30928(15)$ & $0.44155(8)$ & $0.0574(5)$ & $0.7436(17)$ \\
\hline $\mathrm{C} 3 \mathrm{~A}$ & $0.53537(17)$ & $0.33357(18)$ & $0.46817(10)$ & $0.0780(7)$ & $0.7436(17)$ \\
\hline $\mathrm{H} 3 \mathrm{~A}$ & 0.555863 & 0.362570 & 0.498506 & $0.094 *$ & $0.7436(17)$ \\
\hline $\mathrm{C} 4 \mathrm{~A}$ & $0.4606(4)$ & $0.3168(5)$ & $0.4518(3)$ & $0.096(2)$ & $0.7436(17)$ \\
\hline $\mathrm{H} 4 \mathrm{~A}$ & 0.429793 & 0.333886 & 0.470662 & $0.115^{*}$ & $0.7436(17)$ \\
\hline $\mathrm{C} 5 \mathrm{~A}$ & $0.4305(4)$ & $0.2749(5)$ & $0.4078(3)$ & $0.094(3)$ & $0.7436(17)$ \\
\hline $\mathrm{H} 5 \mathrm{~A}$ & 0.379487 & 0.264579 & 0.395771 & $0.113^{*}$ & $0.7436(17)$ \\
\hline C6A & $0.4737(4)$ & $0.2481(5)$ & $0.3813(3)$ & $0.086(2)$ & $0.7436(17)$ \\
\hline H6A & 0.452087 & 0.217872 & 0.351492 & $0.103 *$ & $0.7436(17)$ \\
\hline C7A & $0.5487(4)$ & $0.2652(4)$ & $0.3981(2)$ & $0.0698(15)$ & $0.7436(17)$ \\
\hline H7A & 0.578423 & 0.246450 & 0.379498 & $0.084^{*}$ & $0.7436(17)$ \\
\hline O1B & $0.76424(10)$ & $0.36344(13)$ & $0.60040(7)$ & $0.1001(7)$ & $0.7436(17)$ \\
\hline H1BA & 0.761666 & 0.351068 & 0.570135 & $0.150^{*}$ & $0.2479(6)$ \\
\hline H1BB & 0.749287 & 0.395976 & 0.605330 & $0.150 *$ & $0.2479(6)$ \\
\hline $\mathrm{H} 1 \mathrm{BC}$ & 0.730448 & 0.319412 & 0.615019 & $0.150^{*}$ & $0.2479(6)$ \\
\hline $\mathrm{C} 1 \mathrm{~B}$ & $0.84281(12)$ & $0.38633(15)$ & $0.61999(8)$ & $0.0648(6)$ & $0.7436(17)$ \\
\hline $\mathrm{C} 2 \mathrm{~B}$ & $0.86638(13)$ & $0.32631(14)$ & $0.59957(8)$ & $0.0657(6)$ & $0.7436(17)$ \\
\hline $\mathrm{C} 3 \mathrm{~B}$ & $0.8535(2)$ & $0.3054(2)$ & $0.54985(11)$ & $0.1044(11)$ & $0.7436(17)$ \\
\hline H3B & 0.830275 & 0.328339 & 0.528779 & $0.125^{*}$ & $0.7436(17)$ \\
\hline C4B & $0.8740(5)$ & $0.2516(6)$ & $0.5304(3)$ & $0.119(3)$ & $0.7436(17)$ \\
\hline $\mathrm{H} 4 \mathrm{~B}$ & 0.863199 & 0.236550 & 0.496240 & $0.143^{*}$ & $0.7436(17)$ \\
\hline $\mathrm{C} 5 \mathrm{~B}$ & $0.9095(5)$ & $0.2196(5)$ & $0.5591(3)$ & $0.109(3)$ & $0.7436(17)$ \\
\hline H5B & 0.924530 & 0.183335 & 0.545252 & $0.131^{*}$ & $0.7436(17)$ \\
\hline C6B & $0.9231(4)$ & $0.2407(4)$ & $0.6084(3)$ & $0.093(2)$ & $0.7436(17)$ \\
\hline H6B & 0.947373 & 0.218561 & 0.629241 & $0.112^{*}$ & $0.7436(17)$ \\
\hline $\mathrm{C} 7 \mathrm{~B}$ & $0.9018(2)$ & $0.2941(2)$ & $0.62816(16)$ & $0.0777(9)$ & $0.7436(17)$ \\
\hline H7B & 0.912098 & 0.308569 & 0.662466 & $0.093^{*}$ & $0.7436(17)$ \\
\hline $\mathrm{C} 8 \mathrm{~B}$ & $0.90166(14)$ & $0.47184(15)$ & $0.60289(9)$ & $0.0668(6)$ & $0.7436(17)$ \\
\hline C9B & $0.8772(2)$ & $0.5287(2)$ & $0.59841(10)$ & $0.0883(9)$ & $0.7436(17)$ \\
\hline H9B & 0.822728 & 0.513460 & 0.605400 & $0.106^{*}$ & $0.7436(17)$ \\
\hline C10B & $0.9281(6)$ & $0.6036(5)$ & $0.5846(3)$ & $0.111(3)$ & $0.7436(17)$ \\
\hline H10B & 0.908782 & 0.640297 & 0.580162 & $0.133^{*}$ & $0.7436(17)$ \\
\hline C11B & $1.0077(5)$ & $0.6296(4)$ & $0.5767(3)$ & $0.130(3)$ & $0.7436(17)$ \\
\hline H11B & 1.043803 & 0.684533 & 0.568696 & $0.156^{*}$ & $0.7436(17)$ \\
\hline $\mathrm{C} 12 \mathrm{~B}$ & $1.0361(5)$ & $0.5760(6)$ & $0.5802(4)$ & $0.121(3)$ & $0.7436(17)$ \\
\hline H12B & 1.091060 & 0.592640 & 0.573739 & $0.145^{*}$ & $0.7436(17)$ \\
\hline C13B & $0.9815(3)$ & 0.4974 & $0.59348(16)$ & $0.0874(11)$ & $0.7436(17)$ \\
\hline
\end{tabular}




\begin{tabular}{|c|c|c|c|c|c|}
\hline H13B & 0.999752 & 0.459704 & 0.596214 & $0.105^{*}$ & 0.7436 \\
\hline C14B & 0.83584 (19) & $0.38110(17)$ & $0.67725(10)$ & $0.0612(6)$ & $0.7436(17)$ \\
\hline C15B & $0.77028(16)$ & $0.3162(2)$ & $0.69875(11)$ & $0.0917(9)$ & $0.7436(17)$ \\
\hline H15B & 0.728841 & 0.277251 & 0.677965 & $0.110^{*}$ & $0.7436(17)$ \\
\hline C16B & $0.7637(3)$ & 0.3068 (4) & $0.7494(2)$ & $0.0951(17)$ & $0.7436(17)$ \\
\hline H16B & 0.717770 & 0.261153 & 0.763094 & $0.114^{*}$ & $0.7436(17)$ \\
\hline C17B & 0.8224 & 0.3621 & $0.78128(17)$ & $0.0783(11)$ & $0.7436(17)$ \\
\hline H17B & 0.818095 & 0.355011 & 0.816530 & $0.094 *$ & $0.7436(17)$ \\
\hline C18B & 0.88692 (19) & $0.42746(19)$ & $0.75964(10)$ & $0.0742(8)$ & $0.7436(17)$ \\
\hline H18B & 0.928135 & 0.466765 & 0.780380 & $0.089 *$ & $0.7436(17)$ \\
\hline C19B & 0.8933 (3) & $0.4374(4)$ & $0.7088(2)$ & $0.0655(12)$ & $0.7436(17)$ \\
\hline H19B & 0.938180 & 0.483932 & 0.694994 & $0.079 *$ & $0.7436(17)$ \\
\hline $\mathrm{O} 1 \mathrm{C}$ & 0.666667 & 0.333333 & $0.6432(3)$ & $0.083(3)$ & $0.2564(17)$ \\
\hline $\mathrm{H} 1 \mathrm{C}$ & 0.682226 & 0.379070 & 0.629806 & $0.124^{*}$ & $0.0855(6)$ \\
\hline $\mathrm{C} 1 \mathrm{C}$ & 0.666667 & 0.333333 & $0.6960(4)$ & $0.058(3)$ & $0.2564(17)$ \\
\hline $\mathrm{C} 2 \mathrm{C}$ & $0.7503(5)$ & $0.3564(5)$ & $0.7147(3)$ & $0.073(2)$ & 0.2564 \\
\hline $\mathrm{C} 3 \mathrm{C}$ & $0.7666(11)$ & $0.3251(10)$ & $0.7590(5)$ & $0.113(8)$ & $0.2564(17)$ \\
\hline $\mathrm{H} 3 \mathrm{C}$ & 0.724063 & 0.289629 & 0.780547 & $0.136^{*}$ & $0.2564(17)$ \\
\hline $\mathrm{C} 4 \mathrm{C}$ & $0.8465(10)$ & $0.3481(10)$ & $0.7697(5)$ & $0.117(5)$ & $0.2564(17)$ \\
\hline $\mathrm{H} 4 \mathrm{C}$ & 0.858261 & 0.325808 & 0.798051 & $0.140^{*}$ & $0.2564(17)$ \\
\hline $\mathrm{C} 5 \mathrm{C}$ & $0.9068(7)$ & $0.4009(8)$ & $0.7409(7)$ & $0.149(7)$ & $0.2564(17)$ \\
\hline $\mathrm{H} 5 \mathrm{C}$ & 0.960816 & 0.418783 & 0.750763 & $0.179 *$ & $0.2564(17)$ \\
\hline $\mathrm{C} 6 \mathrm{C}$ & $0.8931(12)$ & $0.4299(17)$ & 0.6977 (9) & $0.124(10)$ & $0.2564(17)$ \\
\hline $\mathrm{H} 6 \mathrm{C}$ & 0.936377 & 0.464379 & 0.676274 & $0.149 *$ & $0.2564(17)$ \\
\hline $\mathrm{C} 7 \mathrm{C}$ & $0.8166(5)$ & 0.4083 (6) & $0.6862(4)$ & $0.094(3)$ & $0.2564(17)$ \\
\hline $\mathrm{H} 7 \mathrm{C}$ & 0.807355 & 0.430151 & 0.656715 & $0.113^{*}$ & $0.2564(17)$ \\
\hline O1D & 0.7440 & 0.4306 & 0.55748 (19) & $0.0885(17)$ & $0.2564(17)$ \\
\hline H1DA & 0.730812 & 0.432220 & 0.587335 & $0.133^{*}$ & $0.0855(6)$ \\
\hline H1DB & 0.761560 & 0.399257 & 0.554882 & $0.133^{*}$ & $0.0855(6)$ \\
\hline H1DC & 0.701323 & 0.413280 & 0.540448 & $0.133^{*}$ & $0.0855(6)$ \\
\hline C1D & 0.8005 (4) & $0.5111(4)$ & $0.5411(2)$ & $0.0642(17)$ & 0.2564 \\
\hline $\mathrm{C} 2 \mathrm{D}$ & $0.7764(3)$ & $0.5690(4)$ & $0.5642(2)$ & $0.0642(18)$ & $0.2564(17)$ \\
\hline $\mathrm{C} 3 \mathrm{D}$ & $0.7632(5)$ & $0.5676(6)$ & 0.6149 (3) & $0.106(3)$ & $0.2564(17)$ \\
\hline H3D & 0.766173 & 0.527914 & 0.634760 & $0.128 *$ & 0.2564 \\
\hline C4D & $0.7462(7)$ & $0.6211(9)$ & $0.6377(5)$ & $0.106(5)$ & 0.2564 \\
\hline H4D & 0.738431 & 0.618454 & 0.672879 & $0.127^{*}$ & 0.2564 \\
\hline C5D & $0.7400(14)$ & 0.6797 (14) & $0.6101(8)$ & $0.108(8)$ & $0.2564(17)$ \\
\hline H5D & 0.729664 & 0.718341 & 0.625539 & $0.129 *$ & $0.2564(17)$ \\
\hline C6D & $0.7497(13)$ & $0.6782(14)$ & $0.5595(8)$ & $0.105(7)$ & $0.2564(17)$ \\
\hline H6D & 0.746374 & 0.717334 & 0.539303 & $0.126^{*}$ & $0.2564(17)$ \\
\hline C7D & $0.7639(18)$ & $0.6222(16)$ & 0.5368 & $0.111(8)$ & $0.2564(17)$ \\
\hline H7D & 0.765172 & 0.620367 & 0.501360 & $0.134 *$ & $0.2564(17)$ \\
\hline $\mathrm{C} 8 \mathrm{D}$ & $0.8844(4)$ & 0.5289 & 0.5587 & $0.0620(17)$ & $0.2564(17)$ \\
\hline C9D & $0.9455(10)$ & $0.6073(9)$ & 0.5784 & $0.089(6)$ & $0.2564(17)$ \\
\hline H9D & 0.932585 & 0.648849 & 0.582298 & $0.107^{*}$ & 0.2564 \\
\hline C10D & $1.0181(9)$ & $0.6216(10)$ & $0.5909(5)$ & $0.095(7)$ & 0.2564 \\
\hline H10D & 1.058560 & 0.673426 & 0.601934 & $0.113 *$ & $0.2564(17)$ \\
\hline C11D & $1.0330(11)$ & $0.5589(12)$ & $0.5873(8)$ & $0.096(8)$ & 0.2564 \\
\hline
\end{tabular}




$\begin{array}{llllll}\text { H11D } & 1.082620 & 0.566820 & 0.600425 & 0.115^{*} & 0.2564(17) \\ \text { C12D } & 0.9796(8) & 0.4827(8) & 0.5654(5) & 0.101(4) & 0.2564(17) \\ \text { H12D } & 0.994971 & 0.443504 & 0.558556 & 0.121^{*} & 0.2564(17) \\ \text { C13D } & 0.9044(5) & 0.4705(4) & 0.5552(4) & 0.098(3) & 0.2564(17) \\ \text { H13D } & 0.863835 & 0.418145 & 0.544928 & 0.118^{*} & 0.2564(17) \\ \text { C14D } & 0.7983(4) & 0.5139(4) & 0.4833(3) & 0.0577(15) & 0.2564(17) \\ \text { C15D } & 0.7290(4) & 0.4634(4) & 0.4585(3) & 0.0679(18) & 0.2564(17) \\ \text { H15D } & 0.682472 & 0.426339 & 0.476747 & 0.081^{*} & 0.2564(17) \\ \text { C16D } & 0.7256(13) & 0.4656(13) & 0.4069(6) & 0.084(7) & 0.2564(17) \\ \text { H16D } & 0.677505 & 0.428401 & 0.390046 & 0.101^{*} & 0.2564(17) \\ \text { C17D } & 0.7909(12) & 0.5208(14) & 0.3795(6) & 0.068(5) & 0.2564(17) \\ \text { H17D } & 0.787198 & 0.524908 & 0.344309 & 0.082^{*} & 0.2564(17) \\ \text { C18D } & 0.8615(12) & 0.5698(12) & 0.4044(6) & 0.073(4) & 0.2564(17) \\ \text { H18D } & 0.908149 & 0.606844 & 0.386238 & 0.087^{*} & 0.2564(17) \\ \text { C19D } & 0.8650(11) & 0.5653(11) & 0.4560(6) & 0.085(5) & 0.2564(17) \\ \text { H19D } & 0.914603 & 0.598511 & 0.472742 & 0.102^{*} & 0.2564(17)\end{array}$

Atomic displacement parameters $\left(\AA^{2}\right)$

\begin{tabular}{|c|c|c|c|c|c|c|}
\hline & $U^{11}$ & $U^{22}$ & $U^{33}$ & $U^{12}$ & $U^{13}$ & $U^{23}$ \\
\hline O1A & $0.1199(16)$ & $0.1199(16)$ & $0.0335(13)$ & $0.0599(8)$ & 0.000 & 0.000 \\
\hline $\mathrm{C} 1 \mathrm{~A}$ & $0.0713(15)$ & $0.0713(15)$ & $0.0329(16)$ & $0.0357(7)$ & 0.000 & 0.000 \\
\hline $\mathrm{C} 2 \mathrm{~A}$ & $0.0639(14)$ & $0.0594(14)$ & $0.0467(12)$ & $0.0293(12)$ & 0.0094 (11) & $0.0053(10)$ \\
\hline $\mathrm{C} 3 \mathrm{~A}$ & $0.0717(18)$ & 0.0823 (19) & $0.0780(17)$ & $0.0371(16)$ & $0.0175(14)$ & $-0.0053(14)$ \\
\hline $\mathrm{C} 4 \mathrm{~A}$ & $0.075(4)$ & $0.092(4)$ & $0.124(4)$ & $0.044(3)$ & $0.027(3)$ & $0.002(3)$ \\
\hline $\mathrm{C} 5 \mathrm{~A}$ & $0.046(2)$ & $0.088(5)$ & $0.133(5)$ & $0.022(2)$ & $0.002(2)$ & $0.016(3)$ \\
\hline C6A & $0.064(3)$ & $0.088(5)$ & $0.095(3)$ & $0.029(3)$ & $-0.013(2)$ & $-0.001(3)$ \\
\hline C7A & $0.072(3)$ & $0.087(3)$ & $0.0562(17)$ & $0.044(2)$ & $-0.005(2)$ & $-0.0042(17)$ \\
\hline O1B & $0.0517(10)$ & 0.1542 (19) & $0.0920(13)$ & $0.0496(11)$ & $-0.0238(9)$ & $-0.0112(12)$ \\
\hline $\mathrm{C} 1 \mathrm{~B}$ & $0.0431(11)$ & $0.0886(16)$ & $0.0611(13)$ & $0.0318(11)$ & $-0.0094(9)$ & $-0.0070(11)$ \\
\hline $\mathrm{C} 2 \mathrm{~B}$ & $0.0477(12)$ & $0.0767(15)$ & $0.0608(13)$ & $0.0221(11)$ & $-0.0055(10)$ & $-0.0089(11)$ \\
\hline C3B & $0.129(3)$ & $0.127(3)$ & $0.0727(18)$ & $0.075(2)$ & $-0.0188(17)$ & $-0.0283(18)$ \\
\hline C4B & $0.164(7)$ & $0.138(6)$ & $0.079(3)$ & $0.093(5)$ & $-0.013(4)$ & -0.039 (4) \\
\hline C5B & $0.126(6)$ & $0.102(5)$ & $0.104(4)$ & $0.062(4)$ & 0.017 (3) & $-0.026(3)$ \\
\hline C6B & 0.103 (4) & $0.100(5)$ & $0.096(4)$ & $0.066(4)$ & $0.002(3)$ & $-0.008(3)$ \\
\hline $\mathrm{C} 7 \mathrm{~B}$ & $0.081(2)$ & $0.086(3)$ & 0.0704 (19) & $0.045(2)$ & $-0.0051(18)$ & -0.0155 (19) \\
\hline C8B & $0.0611(14)$ & $0.0884(17)$ & $0.0549(13)$ & $0.0403(13)$ & $-0.0037(10)$ & $-0.0008(12)$ \\
\hline C9B & $0.106(2)$ & $0.126(3)$ & $0.0599(17)$ & $0.078(2)$ & $0.0093(15)$ & $0.0162(16)$ \\
\hline $\mathrm{C} 10 \mathrm{~B}$ & $0.145(7)$ & $0.133(6)$ & $0.081(3)$ & $0.090(5)$ & $0.025(3)$ & 0.037 (3) \\
\hline $\mathrm{C} 11 \mathrm{~B}$ & $0.157(7)$ & $0.094(4)$ & $0.105(5)$ & $0.036(5)$ & $0.020(4)$ & $0.027(3)$ \\
\hline $\mathrm{C} 12 \mathrm{~B}$ & $0.086(5)$ & $0.100(4)$ & $0.138(7)$ & $0.017(3)$ & $0.024(4)$ & $0.026(4)$ \\
\hline C13B & $0.063(2)$ & $0.082(3)$ & $0.113(3)$ & $0.0326(18)$ & $0.006(2)$ & $0.006(2)$ \\
\hline C14B & 0.0430 & 0.0768 (19) & 0.0647 (14) & 0.0307 (14) & $-0.0015(12)$ & $-0.0041(12)$ \\
\hline C15B & $0.0624(16)$ & $0.099(2)$ & $0.0743(18)$ & $0.0114(15)$ & $0.0047(13)$ & $-0.0138(15)$ \\
\hline C16B & $0.072(3)$ & $0.098(3)$ & $0.078(2)$ & $0.015(2)$ & $0.0179(18)$ & $-0.004(2)$ \\
\hline C17B & $0.087(3)$ & $0.093(3)$ & $0.0624(17)$ & $0.050(2)$ & 0.0047 (17) & $-0.0068(16)$ \\
\hline C18B & 0.0747 (19) & $0.078(2)$ & $0.0666(16)$ & $0.0360(16)$ & $-0.0144(13)$ & $-0.0065(14)$ \\
\hline C19B & $0.055(2)$ & $0.075(3)$ & $0.0624(18)$ & $0.029(2)$ & $-0.0078(15)$ & $-0.0024(17)$ \\
\hline
\end{tabular}


supporting information

$\begin{array}{lllllll}\text { O1C } & 0.105(4) & 0.105(4) & 0.039(4) & 0.052(2) & 0.000 & 0.000 \\ \text { C1C } & 0.066(4) & 0.066(4) & 0.041(5) & 0.033(2) & 0.000 & 0.000 \\ \text { C2C } & 0.095(6) & 0.080(5) & 0.059(4) & 0.054(5) & 0.001(4) & 0.007(4) \\ \text { C3C } & 0.146(15) & 0.121(14) & 0.081(10) & 0.074(12) & -0.016(9) & 0.034(10) \\ \text { C4C } & 0.140(15) & 0.119(11) & 0.108(11) & 0.077(11) & -0.046(10) & 0.011(9) \\ \text { C5C } & 0.081(8) & 0.083(8) & 0.28(2) & 0.040(7) & -0.022(11) & 0.012(11) \\ \text { C6C } & 0.115(16) & 0.126(18) & 0.15(2) & 0.074(15) & 0.021(14) & 0.042(16) \\ \text { C7C } & 0.072(6) & 0.108(8) & 0.121(8) & 0.059(6) & 0.024(5) & 0.041(6) \\ \text { O1D } & 0.073(3) & 0.061(3) & 0.097(4) & 0.008(2) & -0.007(3) & 0.031(3) \\ \text { C1D } & 0.054(4) & 0.047(3) & 0.073(4) & 0.012(3) & -0.001(3) & 0.017(3) \\ \text { C2D } & 0.041(3) & 0.067(4) & 0.070(4) & 0.015(3) & 0.008(3) & 0.020(3) \\ \text { C3D } & 0.124(8) & 0.133(8) & 0.082(5) & 0.078(6) & 0.051(5) & 0.046(5) \\ \text { C4D } & 0.101(10) & 0.162(13) & 0.087(8) & 0.091(10) & 0.026(7) & 0.014(8) \\ \text { C5D } & 0.082(10) & 0.19(2) & 0.087(11) & 0.091(12) & -0.019(7) & -0.024(11) \\ \text { C6D } & 0.124(18) & 0.110(14) & 0.097(12) & 0.072(13) & -0.006(11) & 0.011(10) \\ \text { C7D } & 0.126(13) & 0.149(19) & 0.101(12) & 0.100(14) & -0.003(9) & -0.020(11) \\ \text { C8D } & 0.055(4) & 0.059(4) & 0.065(4) & 0.023(3) & 0.006(3) & 0.011(3) \\ \text { C9D } & 0.044(5) & 0.063(7) & 0.133(15) & 0.005(5) & 0.001(7) & 0.025(7) \\ \text { C10D } & 0.060(6) & 0.162(17) & 0.056(6) & 0.052(8) & -0.010(5) & -0.050(8) \\ \text { C11D } & 0.072(13) & 0.17(3) & 0.057(6) & 0.071(17) & 0.001(7) & 0.005(10) \\ \text { C12D } & 0.111(9) & 0.101(8) & 0.115(9) & 0.071(7) & -0.034(8) & -0.014(7) \\ \text { C13D } & 0.080(6) & 0.057(4) & 0.152(9) & 0.030(4) & -0.026(5) & -0.001(5) \\ \text { C14D } & 0.052(4) & 0.040(3) & 0.083(4) & 0.024(3) & -0.003(3) & -0.001(3) \\ \text { C15D } & 0.058(4) & 0.053(4) & 0.084(5) & 0.021(3) & 0.000(4) & -0.002(4) \\ \text { C16D } & 0.071(8) & 0.077(9) & 0.114(14) & 0.044(7) & -0.033(8) & -0.027(8) \\ \text { C17D } & 0.093(11) & 0.084(9) & 0.055(5) & 0.065(9) & -0.006(5) & -0.010(5) \\ \text { C18D } & 0.072(7) & 0.097(9) & 0.068(7) & 0.056(7) & 0.004(5) & 0.007(6) \\ \text { C19D } & 0.075(8) & 0.084(12) & 0.082(9) & 0.029(7) & -0.002(6) & 0.027(8)\end{array}$

Geometric parameters ( $\left.\AA,{ }^{\circ}\right)$

\begin{tabular}{llll}
\hline $\mathrm{O} 1 \mathrm{~A}-\mathrm{C} 1 \mathrm{~A}$ & $1.434(4)$ & $\mathrm{O} 1 \mathrm{C}-\mathrm{C} 1 \mathrm{C}$ & $1.411(11)$ \\
$\mathrm{O} 1 \mathrm{~A}-\mathrm{H} 1 \mathrm{~A}$ & 0.8549 & $\mathrm{O} 1 \mathrm{C}-\mathrm{H} 1 \mathrm{C}$ & 0.8500 \\
$\mathrm{O} 1 \mathrm{~A}-\mathrm{H} 1 \mathrm{~A}^{\mathrm{i}}$ & 0.8549 & $\mathrm{O} 1 \mathrm{C}-\mathrm{H} 1 \mathrm{C}^{\mathrm{i}}$ & 0.8500 \\
$\mathrm{O} 1 \mathrm{~A}-\mathrm{H} 1 \mathrm{~A}^{\mathrm{ii}}$ & 0.8549 & $\mathrm{O} 1 \mathrm{C}-\mathrm{H} 1 \mathrm{C}$ ii & $1.517(8)$ \\
$\mathrm{C} 1 \mathrm{~A}-\mathrm{C} 2 \mathrm{~A}$ & $1.524(3)$ & $\mathrm{C} 1 \mathrm{C}-\mathrm{C} 2 \mathrm{C}$ & $1.518(8)$ \\
$\mathrm{C} 1 \mathrm{~A}-\mathrm{C} 2 \mathrm{~A}^{\mathrm{ii}}$ & $1.524(3)$ & $\mathrm{C} 1 \mathrm{C}-\mathrm{C} 2 \mathrm{C}^{\mathrm{ii}}$ & $1.518(8)$ \\
$\mathrm{C} 1 \mathrm{~A}-\mathrm{C} 2 \mathrm{~A}^{\mathrm{i}}$ & $1.524(3)$ & $\mathrm{C} 1 \mathrm{C}-\mathrm{C} 2 \mathrm{C}^{\mathrm{i}}$ & $1.384(10)$ \\
$\mathrm{C} 2 \mathrm{~A}-\mathrm{C} 3 \mathrm{~A}$ & $1.388(3)$ & $\mathrm{C} 2 \mathrm{C}-\mathrm{C} 7 \mathrm{C}$ & $1.430(14)$ \\
$\mathrm{C} 2 \mathrm{~A}-\mathrm{C} 7 \mathrm{~A}$ & $1.389(6)$ & $\mathrm{C} 2 \mathrm{C}-\mathrm{C} 3 \mathrm{C}$ & $1.394(17)$ \\
$\mathrm{C} 3 \mathrm{~A}-\mathrm{C} 4 \mathrm{~A}$ & $1.373(7)$ & $\mathrm{C} 3 \mathrm{C}-\mathrm{C} 4 \mathrm{C}$ & 0.9500 \\
$\mathrm{C} 3 \mathrm{~A}-\mathrm{H} 3 \mathrm{~A}$ & 0.9500 & $\mathrm{C} 3 \mathrm{C}-\mathrm{H} 3 \mathrm{C}$ & $1.335(15)$ \\
$\mathrm{C} 4 \mathrm{~A}-\mathrm{C} 5 \mathrm{~A}$ & $1.377(9)$ & $\mathrm{C} 4 \mathrm{C}-\mathrm{C} 5 \mathrm{C}$ & 0.9500 \\
$\mathrm{C} 4 \mathrm{~A}-\mathrm{H} 4 \mathrm{~A}$ & 0.9500 & $\mathrm{C} 4 \mathrm{C}-\mathrm{H} 4 \mathrm{C}$ & $1.363(18)$ \\
$\mathrm{C} 5 \mathrm{~A}-\mathrm{C} 6 \mathrm{~A}$ & $1.368(8)$ & $\mathrm{C} 5 \mathrm{C}-\mathrm{C} 6 \mathrm{C}$ & 0.9500 \\
$\mathrm{C} 5 \mathrm{~A}-\mathrm{H} 5 \mathrm{~A}$ & 0.9500 & $\mathrm{C} 5 \mathrm{C}-\mathrm{H} 5 \mathrm{C}$ & $1.343(17)$ \\
$\mathrm{C} 6 \mathrm{~A}-\mathrm{C} 7 \mathrm{~A}$ & $1.379(7)$ & $\mathrm{C} 6 \mathrm{C}-\mathrm{C} 7 \mathrm{C}$ & 0.9500 \\
$\mathrm{C} 6 \mathrm{~A}-\mathrm{H} 6 \mathrm{~A}$ & 0.9500 & $\mathrm{C} 6 \mathrm{C}-\mathrm{H} 6 \mathrm{C}$ &
\end{tabular}




\begin{tabular}{|c|c|c|c|}
\hline $\mathrm{C} 7 \mathrm{~A}-\mathrm{H} 7 \mathrm{~A}$ & 0.9500 & $\mathrm{C} 7 \mathrm{C}-\mathrm{H} 7 \mathrm{C}$ & 0.9500 \\
\hline $\mathrm{O} 1 \mathrm{~B}-\mathrm{C} 1 \mathrm{~B}$ & $1.438(2)$ & $\mathrm{O} 1 \mathrm{D}-\mathrm{C} 1 \mathrm{D}$ & $1.438(7)$ \\
\hline $\mathrm{O} 1 \mathrm{~B}-\mathrm{H} 1 \mathrm{BA}$ & 0.8376 & $\mathrm{O} 1 \mathrm{D}-\mathrm{H} 1 \mathrm{DA}$ & 0.8426 \\
\hline $\mathrm{O} 1 \mathrm{~B}-\mathrm{H} 1 \mathrm{BB}$ & 0.8158 & $\mathrm{O} 1 \mathrm{D}-\mathrm{H} 1 \mathrm{DB}$ & 0.8241 \\
\hline $\mathrm{O} 1 \mathrm{~B}-\mathrm{H} 1 \mathrm{BC}$ & 0.8580 & $\mathrm{O} 1 \mathrm{D}-\mathrm{H} 1 \mathrm{DC}$ & 0.8453 \\
\hline $\mathrm{C} 1 \mathrm{~B}-\mathrm{C} 8 \mathrm{~B}$ & $1.521(3)$ & $\mathrm{C} 1 \mathrm{D}-\mathrm{C} 2 \mathrm{D}$ & $1.527(9)$ \\
\hline $\mathrm{C} 1 \mathrm{~B}-\mathrm{C} 2 \mathrm{~B}$ & $1.530(3)$ & $\mathrm{C} 1 \mathrm{D}-\mathrm{C} 8 \mathrm{D}$ & $1.540(9)$ \\
\hline $\mathrm{C} 1 \mathrm{~B}-\mathrm{C} 14 \mathrm{~B}$ & $1.536(3)$ & $\mathrm{C} 1 \mathrm{D}-\mathrm{C} 14 \mathrm{D}$ & $1.548(9)$ \\
\hline $\mathrm{C} 2 \mathrm{~B}-\mathrm{C} 7 \mathrm{~B}$ & $1.357(5)$ & $\mathrm{C} 2 \mathrm{D}-\mathrm{C} 7 \mathrm{D}$ & $1.368(16)$ \\
\hline $\mathrm{C} 2 \mathrm{~B}-\mathrm{C} 3 \mathrm{~B}$ & $1.374(3)$ & $\mathrm{C} 2 \mathrm{D}-\mathrm{C} 3 \mathrm{D}$ & $1.379(9)$ \\
\hline $\mathrm{C} 3 \mathrm{~B}-\mathrm{C} 4 \mathrm{~B}$ & $1.375(8)$ & $\mathrm{C} 3 \mathrm{D}-\mathrm{C} 4 \mathrm{D}$ & $1.364(13)$ \\
\hline $\mathrm{C} 3 \mathrm{~B}-\mathrm{H} 3 \mathrm{~B}$ & 0.9500 & $\mathrm{C} 3 \mathrm{D}-\mathrm{H} 3 \mathrm{D}$ & 0.9500 \\
\hline $\mathrm{C} 4 \mathrm{~B}-\mathrm{C} 5 \mathrm{~B}$ & $1.357(11)$ & $C 4 D-C 5 D$ & $1.40(2)$ \\
\hline $\mathrm{C} 4 \mathrm{~B}-\mathrm{H} 4 \mathrm{~B}$ & 0.9500 & $\mathrm{C} 4 \mathrm{D}-\mathrm{H} 4 \mathrm{D}$ & 0.9500 \\
\hline $\mathrm{C} 5 \mathrm{~B}-\mathrm{C} 6 \mathrm{~B}$ & $1.364(6)$ & $\mathrm{C} 5 \mathrm{D}-\mathrm{C} 6 \mathrm{D}$ & $1.368(15)$ \\
\hline $\mathrm{C} 5 \mathrm{~B}-\mathrm{H} 5 \mathrm{~B}$ & 0.9500 & $\mathrm{C} 5 \mathrm{D}-\mathrm{H} 5 \mathrm{D}$ & 0.9500 \\
\hline $\mathrm{C} 6 \mathrm{~B}-\mathrm{C} 7 \mathrm{~B}$ & $1.381(7)$ & C6D-C7D & $1.37(2)$ \\
\hline $\mathrm{C} 6 \mathrm{~B}-\mathrm{H} 6 \mathrm{~B}$ & 0.9500 & C6D-H6D & 0.9500 \\
\hline $\mathrm{C} 7 \mathrm{~B}-\mathrm{H} 7 \mathrm{~B}$ & 0.9500 & C7D-H7D & 0.9500 \\
\hline $\mathrm{C} 8 \mathrm{~B}-\mathrm{C} 13 \mathrm{~B}$ & $1.374(5)$ & $\mathrm{C} 8 \mathrm{D}-\mathrm{C} 13 \mathrm{D}$ & $1.354(9)$ \\
\hline $\mathrm{C} 8 \mathrm{~B}-\mathrm{C} 9 \mathrm{~B}$ & $1.389(4)$ & $\mathrm{C} 8 \mathrm{D}-\mathrm{C} 9 \mathrm{D}$ & $1.463(15)$ \\
\hline $\mathrm{C} 9 \mathrm{~B}-\mathrm{C} 10 \mathrm{~B}$ & $1.320(8)$ & $\mathrm{C} 9 \mathrm{D}-\mathrm{C} 10 \mathrm{D}$ & $1.318(17)$ \\
\hline C9B-H9B & 0.9500 & C9D-H9D & 0.9500 \\
\hline $\mathrm{C} 10 \mathrm{~B}-\mathrm{C} 11 \mathrm{~B}$ & $1.364(9)$ & $\mathrm{C} 10 \mathrm{D}-\mathrm{C} 11 \mathrm{D}$ & $1.37(2)$ \\
\hline $\mathrm{C} 10 \mathrm{~B}-\mathrm{H} 10 \mathrm{~B}$ & 0.9500 & $\mathrm{C} 10 \mathrm{D}-\mathrm{H} 10 \mathrm{D}$ & 0.9500 \\
\hline $\mathrm{C} 11 \mathrm{~B}-\mathrm{C} 12 \mathrm{~B}$ & $1.382(11)$ & $\mathrm{C} 11 \mathrm{D}-\mathrm{C} 12 \mathrm{D}$ & $1.423(18)$ \\
\hline $\mathrm{C} 11 \mathrm{~B}-\mathrm{H} 11 \mathrm{~B}$ & 0.9500 & C11D-H11D & 0.9500 \\
\hline $\mathrm{C} 12 \mathrm{~B}-\mathrm{C} 13 \mathrm{~B}$ & $1.382(10)$ & $\mathrm{C} 12 \mathrm{D}-\mathrm{C} 13 \mathrm{D}$ & $1.367(12)$ \\
\hline $\mathrm{C} 12 \mathrm{~B}-\mathrm{H} 12 \mathrm{~B}$ & 0.9500 & $\mathrm{C} 12 \mathrm{D}-\mathrm{H} 12 \mathrm{D}$ & 0.9500 \\
\hline $\mathrm{C} 13 \mathrm{~B}-\mathrm{H} 13 \mathrm{~B}$ & 0.9500 & $\mathrm{C} 13 \mathrm{D}-\mathrm{H} 13 \mathrm{D}$ & 0.9500 \\
\hline $\mathrm{C} 14 \mathrm{~B}-\mathrm{C} 15 \mathrm{~B}$ & $1.374(4)$ & $\mathrm{C} 14 \mathrm{D}-\mathrm{C} 15 \mathrm{D}$ & $1.361(9)$ \\
\hline $\mathrm{C} 14 \mathrm{~B}-\mathrm{C} 19 \mathrm{~B}$ & $1.376(7)$ & $\mathrm{C} 14 \mathrm{D}-\mathrm{C} 19 \mathrm{D}$ & $1.369(16)$ \\
\hline $\mathrm{C} 15 \mathrm{~B}-\mathrm{C} 16 \mathrm{~B}$ & $1.363(6)$ & $\mathrm{C} 15 \mathrm{D}-\mathrm{C} 16 \mathrm{D}$ & $1.383(16)$ \\
\hline $\mathrm{C} 15 \mathrm{~B}-\mathrm{H} 15 \mathrm{~B}$ & 0.9500 & $\mathrm{C} 15 \mathrm{D}-\mathrm{H} 15 \mathrm{D}$ & 0.9500 \\
\hline $\mathrm{C} 16 \mathrm{~B}-\mathrm{C} 17 \mathrm{~B}$ & $1.386(7)$ & $\mathrm{C} 16 \mathrm{D}-\mathrm{C} 17 \mathrm{D}$ & $1.376(18)$ \\
\hline $\mathrm{C} 16 \mathrm{~B}-\mathrm{H} 16 \mathrm{~B}$ & 0.9500 & $\mathrm{C} 16 \mathrm{D}-\mathrm{H} 16 \mathrm{D}$ & 0.9500 \\
\hline $\mathrm{C} 17 \mathrm{~B}-\mathrm{C} 18 \mathrm{~B}$ & $1.371(5)$ & $\mathrm{C} 17 \mathrm{D}-\mathrm{C} 18 \mathrm{D}$ & $1.371(18)$ \\
\hline $\mathrm{C} 17 \mathrm{~B}-\mathrm{H} 17 \mathrm{~B}$ & 0.9500 & $\mathrm{C} 17 \mathrm{D}-\mathrm{H} 17 \mathrm{D}$ & 0.9500 \\
\hline $\mathrm{C} 18 \mathrm{~B}-\mathrm{C} 19 \mathrm{~B}$ & $1.370(7)$ & $\mathrm{C} 18 \mathrm{D}-\mathrm{C} 19 \mathrm{D}$ & $1.386(17)$ \\
\hline $\mathrm{C} 18 \mathrm{~B}-\mathrm{H} 18 \mathrm{~B}$ & 0.9500 & $\mathrm{C} 18 \mathrm{D}-\mathrm{H} 18 \mathrm{D}$ & 0.9500 \\
\hline C19B-H19B & 0.9500 & C19D-H19D & 0.9500 \\
\hline $\mathrm{C} 1 \mathrm{~A}-\mathrm{O} 1 \mathrm{~A}-\mathrm{H} 1 \mathrm{~A}$ & 112.8 & $\mathrm{C} 14 \mathrm{~B}-\mathrm{C} 19 \mathrm{~B}-\mathrm{H} 19 \mathrm{~B}$ & 119.4 \\
\hline $\mathrm{C} 1 \mathrm{~A}-\mathrm{O} 1 \mathrm{~A}-\mathrm{H} 1 \mathrm{~A}^{\mathrm{i}}$ & 112.8 & $\mathrm{C} 1 \mathrm{C}-\mathrm{O} 1 \mathrm{C}-\mathrm{H} 1 \mathrm{C}$ & 114.9 \\
\hline $\mathrm{C} 1 \mathrm{~A}-\mathrm{O} 1 \mathrm{~A}-\mathrm{H} 1 \mathrm{~A}^{\mathrm{ii}}$ & $112.754(1)$ & $\mathrm{C} 1 \mathrm{C}-\mathrm{O} 1 \mathrm{C}-\mathrm{H} 1 \mathrm{C}^{\mathrm{i}}$ & $114.914(3)$ \\
\hline $\mathrm{H} 1 \mathrm{~A}-\mathrm{O} 1 \mathrm{~A}-\mathrm{H} 1 \mathrm{~A}^{\mathrm{i}}$ & 106.0 & $\mathrm{H} 1 \mathrm{C}-\mathrm{O} 1 \mathrm{C}-\mathrm{H} 1 \mathrm{C}^{\mathrm{i}}$ & 103.5 \\
\hline $\mathrm{H} 1 \mathrm{~A}-\mathrm{O} 1 \mathrm{~A}-\mathrm{H} 1 \mathrm{~A}^{\mathrm{ii}}$ & 106.0 & $\mathrm{O} 1 \mathrm{C}-\mathrm{C} 1 \mathrm{C}-\mathrm{C} 2 \mathrm{C}$ & $109.3(4)$ \\
\hline $\mathrm{H} 1 \mathrm{~A}-\mathrm{O} 1 \mathrm{~A}-\mathrm{H} 1 \mathrm{~A}^{\mathrm{ii}}$ & 106.0 & $\mathrm{O} 1 \mathrm{C}-\mathrm{C} 1 \mathrm{C}-\mathrm{C} 2 \mathrm{C}^{\mathrm{ii}}$ & $109.3(4)$ \\
\hline
\end{tabular}




\begin{tabular}{|c|c|}
\hline $\mathrm{O} 1 \mathrm{~A}-\mathrm{C} 1 \mathrm{~A}-\mathrm{C} 2 \mathrm{~A}$ & $107.99(14)$ \\
\hline $\mathrm{O} 1 \mathrm{~A}-\mathrm{C} 1 \mathrm{~A}-\mathrm{C} 2 \mathrm{~A}^{\mathrm{ii}}$ & $107.99(14)$ \\
\hline $\mathrm{C} 2 \mathrm{~A}-\mathrm{C} 1 \mathrm{~A}-\mathrm{C} 2 \mathrm{~A}^{\mathrm{ii}}$ & $110.91(13)$ \\
\hline $\mathrm{O} 1 \mathrm{~A}-\mathrm{C} 1 \mathrm{~A}-\mathrm{C} 2 \mathrm{~A}^{\mathrm{i}}$ & $107.99(14)$ \\
\hline $\mathrm{C} 2 \mathrm{~A}-\mathrm{C} 1 \mathrm{~A}-\mathrm{C} 2 \mathrm{~A}^{\mathrm{i}}$ & $110.91(13)$ \\
\hline $\mathrm{C} 2 \mathrm{~A}^{\mathrm{ii}}-\mathrm{C} 1 \mathrm{~A}-\mathrm{C} 2 \mathrm{~A}^{\mathrm{i}}$ & $110.90(13)$ \\
\hline $\mathrm{C} 3 \mathrm{~A}-\mathrm{C} 2 \mathrm{~A}-\mathrm{C} 7 \mathrm{~A}$ & $117.3(4)$ \\
\hline $\mathrm{C} 3 \mathrm{~A}-\mathrm{C} 2 \mathrm{~A}-\mathrm{C} 1 \mathrm{~A}$ & $120.3(2)$ \\
\hline $\mathrm{C} 7 \mathrm{~A}-\mathrm{C} 2 \mathrm{~A}-\mathrm{C} 1 \mathrm{~A}$ & $122.4(4)$ \\
\hline $\mathrm{C} 4 \mathrm{~A}-\mathrm{C} 3 \mathrm{~A}-\mathrm{C} 2 \mathrm{~A}$ & $121.7(4)$ \\
\hline $\mathrm{C} 4 \mathrm{~A}-\mathrm{C} 3 \mathrm{~A}-\mathrm{H} 3 \mathrm{~A}$ & 119.1 \\
\hline $\mathrm{C} 2 \mathrm{~A}-\mathrm{C} 3 \mathrm{~A}-\mathrm{H} 3 \mathrm{~A}$ & 119.1 \\
\hline $\mathrm{C} 3 \mathrm{~A}-\mathrm{C} 4 \mathrm{~A}-\mathrm{C} 5 \mathrm{~A}$ & $119.5(6)$ \\
\hline $\mathrm{C} 3 \mathrm{~A}-\mathrm{C} 4 \mathrm{~A}-\mathrm{H} 4 \mathrm{~A}$ & 120.2 \\
\hline $\mathrm{C} 5 \mathrm{~A}-\mathrm{C} 4 \mathrm{~A}-\mathrm{H} 4 \mathrm{~A}$ & 120.2 \\
\hline $\mathrm{C} 6 \mathrm{~A}-\mathrm{C} 5 \mathrm{~A}-\mathrm{C} 4 \mathrm{~A}$ & $120.3(6)$ \\
\hline $\mathrm{C} 6 \mathrm{~A}-\mathrm{C} 5 \mathrm{~A}-\mathrm{H} 5 \mathrm{~A}$ & 119.8 \\
\hline $\mathrm{C} 4 \mathrm{~A}-\mathrm{C} 5 \mathrm{~A}-\mathrm{H} 5 \mathrm{~A}$ & 119.8 \\
\hline $\mathrm{C} 5 \mathrm{~A}-\mathrm{C} 6 \mathrm{~A}-\mathrm{C} 7 \mathrm{~A}$ & $119.7(7)$ \\
\hline $\mathrm{C} 5 \mathrm{~A}-\mathrm{C} 6 \mathrm{~A}-\mathrm{H} 6 \mathrm{~A}$ & 120.1 \\
\hline $\mathrm{C} 7 \mathrm{~A}-\mathrm{C} 6 \mathrm{~A}-\mathrm{H} 6 \mathrm{~A}$ & 120.1 \\
\hline $\mathrm{C} 6 \mathrm{~A}-\mathrm{C} 7 \mathrm{~A}-\mathrm{C} 2 \mathrm{~A}$ & $121.4(7)$ \\
\hline $\mathrm{C} 6 \mathrm{~A}-\mathrm{C} 7 \mathrm{~A}-\mathrm{H} 7 \mathrm{~A}$ & 119.3 \\
\hline $\mathrm{C} 2 \mathrm{~A}-\mathrm{C} 7 \mathrm{~A}-\mathrm{H} 7 \mathrm{~A}$ & 119.3 \\
\hline $\mathrm{C} 1 \mathrm{~B}-\mathrm{O} 1 \mathrm{~B}-\mathrm{H} 1 \mathrm{BA}$ & 110.1 \\
\hline $\mathrm{C} 1 \mathrm{~B}-\mathrm{O} 1 \mathrm{~B}-\mathrm{H} 1 \mathrm{BB}$ & 114.9 \\
\hline $\mathrm{C} 1 \mathrm{~B}-\mathrm{O} 1 \mathrm{~B}-\mathrm{H} 1 \mathrm{BC}$ & 107.0 \\
\hline $\mathrm{H} 1 \mathrm{BA}-\mathrm{O} 1 \mathrm{~B}-\mathrm{H} 1 \mathrm{BB}$ & 112.2 \\
\hline $\mathrm{H} 1 \mathrm{BA}-\mathrm{O} 1 \mathrm{~B}-\mathrm{H} 1 \mathrm{BC}$ & 104.7 \\
\hline $\mathrm{H} 1 \mathrm{BB}-\mathrm{O} 1 \mathrm{~B}-\mathrm{H} 1 \mathrm{BC}$ & 107.2 \\
\hline $\mathrm{O} 1 \mathrm{~B}-\mathrm{C} 1 \mathrm{~B}-\mathrm{C} 8 \mathrm{~B}$ & $108.49(19)$ \\
\hline $\mathrm{O} 1 \mathrm{~B}-\mathrm{C} 1 \mathrm{~B}-\mathrm{C} 2 \mathrm{~B}$ & $107.29(18)$ \\
\hline $\mathrm{C} 8 \mathrm{~B}-\mathrm{C} 1 \mathrm{~B}-\mathrm{C} 2 \mathrm{~B}$ & $111.28(18)$ \\
\hline $\mathrm{O} 1 \mathrm{~B}-\mathrm{C} 1 \mathrm{~B}-\mathrm{C} 14 \mathrm{~B}$ & $107.45(19)$ \\
\hline $\mathrm{C} 8 \mathrm{~B}-\mathrm{C} 1 \mathrm{~B}-\mathrm{C} 14 \mathrm{~B}$ & $111.3(2)$ \\
\hline $\mathrm{C} 2 \mathrm{~B}-\mathrm{C} 1 \mathrm{~B}-\mathrm{C} 14 \mathrm{~B}$ & $110.8(2)$ \\
\hline $\mathrm{C} 7 \mathrm{~B}-\mathrm{C} 2 \mathrm{~B}-\mathrm{C} 3 \mathrm{~B}$ & $118.1(3)$ \\
\hline $\mathrm{C} 7 \mathrm{~B}-\mathrm{C} 2 \mathrm{~B}-\mathrm{C} 1 \mathrm{~B}$ & $122.8(2)$ \\
\hline $\mathrm{C} 3 \mathrm{~B}-\mathrm{C} 2 \mathrm{~B}-\mathrm{C} 1 \mathrm{~B}$ & $119.1(2)$ \\
\hline $\mathrm{C} 2 \mathrm{~B}-\mathrm{C} 3 \mathrm{~B}-\mathrm{C} 4 \mathrm{~B}$ & $120.4(5)$ \\
\hline $\mathrm{C} 2 \mathrm{~B}-\mathrm{C} 3 \mathrm{~B}-\mathrm{H} 3 \mathrm{~B}$ & 119.8 \\
\hline $\mathrm{C} 4 \mathrm{~B}-\mathrm{C} 3 \mathrm{~B}-\mathrm{H} 3 \mathrm{~B}$ & 119.8 \\
\hline $\mathrm{C} 5 \mathrm{~B}-\mathrm{C} 4 \mathrm{~B}-\mathrm{C} 3 \mathrm{~B}$ & $121.3(6)$ \\
\hline $\mathrm{C} 5 \mathrm{~B}-\mathrm{C} 4 \mathrm{~B}-\mathrm{H} 4 \mathrm{~B}$ & 119.4 \\
\hline $\mathrm{C} 3 \mathrm{~B}-\mathrm{C} 4 \mathrm{~B}-\mathrm{H} 4 \mathrm{~B}$ & 119.4 \\
\hline $\mathrm{C} 4 \mathrm{~B}-\mathrm{C} 5 \mathrm{~B}-\mathrm{C} 6 \mathrm{~B}$ & $118.5(7)$ \\
\hline $\mathrm{C} 4 \mathrm{~B}-\mathrm{C} 5 \mathrm{~B}-\mathrm{H} 5 \mathrm{~B}$ & 120.7 \\
\hline & \\
\hline
\end{tabular}

\begin{tabular}{|c|c|}
\hline $\mathrm{C} 2 \mathrm{C}-\mathrm{C} 1 \mathrm{C}-\mathrm{C} 2 \mathrm{C}$ & $109.6(4)$ \\
\hline $\mathrm{O} 1 \mathrm{C}-\mathrm{C} 1 \mathrm{C}-\mathrm{C} 2 \mathrm{C}^{\mathrm{i}}$ & $109.3(4)$ \\
\hline $\mathrm{C} 2 \mathrm{C}-\mathrm{C} 1 \mathrm{C}-\mathrm{C} 2 \mathrm{C}^{\mathrm{i}}$ & $109.6(4)$ \\
\hline $\mathrm{C} 2 \mathrm{C}^{\mathrm{ii}}-\mathrm{C} 1 \mathrm{C}-\mathrm{C} 2 \mathrm{C}^{\mathrm{i}}$ & $109.6(4)$ \\
\hline $\mathrm{C} 7 \mathrm{C}-\mathrm{C} 2 \mathrm{C}-\mathrm{C} 3 \mathrm{C}$ & $116.3(10)$ \\
\hline $\mathrm{C} 7 \mathrm{C}-\mathrm{C} 2 \mathrm{C}-\mathrm{C} 1 \mathrm{C}$ & $118.8(7)$ \\
\hline $\mathrm{C} 3 \mathrm{C}-\mathrm{C} 2 \mathrm{C}-\mathrm{C} 1 \mathrm{C}$ & $124.8(10)$ \\
\hline $\mathrm{C} 4 \mathrm{C}-\mathrm{C} 3 \mathrm{C}-\mathrm{C} 2 \mathrm{C}$ & $118.2(13)$ \\
\hline $\mathrm{C} 4 \mathrm{C}-\mathrm{C} 3 \mathrm{C}-\mathrm{H} 3 \mathrm{C}$ & 120.9 \\
\hline $\mathrm{C} 2 \mathrm{C}-\mathrm{C} 3 \mathrm{C}-\mathrm{H} 3 \mathrm{C}$ & 120.9 \\
\hline $\mathrm{C} 5 \mathrm{C}-\mathrm{C} 4 \mathrm{C}-\mathrm{C} 3 \mathrm{C}$ & $121.1(12)$ \\
\hline $\mathrm{C} 5 \mathrm{C}-\mathrm{C} 4 \mathrm{C}-\mathrm{H} 4 \mathrm{C}$ & 119.4 \\
\hline $\mathrm{C} 3 \mathrm{C}-\mathrm{C} 4 \mathrm{C}-\mathrm{H} 4 \mathrm{C}$ & 119.4 \\
\hline $\mathrm{C} 4 \mathrm{C}-\mathrm{C} 5 \mathrm{C}-\mathrm{C} 6 \mathrm{C}$ & $121.9(13)$ \\
\hline $\mathrm{C} 4 \mathrm{C}-\mathrm{C} 5 \mathrm{C}-\mathrm{H} 5 \mathrm{C}$ & 119.0 \\
\hline $\mathrm{C} 6 \mathrm{C}-\mathrm{C} 5 \mathrm{C}-\mathrm{H} 5 \mathrm{C}$ & 119.0 \\
\hline $\mathrm{C} 7 \mathrm{C}-\mathrm{C} 6 \mathrm{C}-\mathrm{C} 5 \mathrm{C}$ & $118.0(17)$ \\
\hline $\mathrm{C} 7 \mathrm{C}-\mathrm{C} 6 \mathrm{C}-\mathrm{H} 6 \mathrm{C}$ & 121.0 \\
\hline $\mathrm{C} 5 \mathrm{C}-\mathrm{C} 6 \mathrm{C}-\mathrm{H} 6 \mathrm{C}$ & 121.0 \\
\hline $\mathrm{C} 6 \mathrm{C}-\mathrm{C} 7 \mathrm{C}-\mathrm{C} 2 \mathrm{C}$ & $124.2(12)$ \\
\hline $\mathrm{C} 6 \mathrm{C}-\mathrm{C} 7 \mathrm{C}-\mathrm{H} 7 \mathrm{C}$ & 117.9 \\
\hline $\mathrm{C} 2 \mathrm{C}-\mathrm{C} 7 \mathrm{C}-\mathrm{H} 7 \mathrm{C}$ & 117.9 \\
\hline $\mathrm{C} 1 \mathrm{D}-\mathrm{O} 1 \mathrm{D}-\mathrm{H} 1 \mathrm{DA}$ & 109.2 \\
\hline $\mathrm{C} 1 \mathrm{D}-\mathrm{O} 1 \mathrm{D}-\mathrm{H} 1 \mathrm{DB}$ & 113.2 \\
\hline $\mathrm{C} 1 \mathrm{D}-\mathrm{O} 1 \mathrm{D}-\mathrm{H} 1 \mathrm{DC}$ & 108.5 \\
\hline $\mathrm{H} 1 \mathrm{DA}-\mathrm{O} 1 \mathrm{D}-\mathrm{H} 1 \mathrm{DB}$ & 110.2 \\
\hline $\mathrm{H} 1 \mathrm{DA}-\mathrm{O} 1 \mathrm{D}-\mathrm{H} 1 \mathrm{DC}$ & 106.0 \\
\hline $\mathrm{H} 1 \mathrm{DB}-\mathrm{O} 1 \mathrm{D}-\mathrm{H} 1 \mathrm{DC}$ & 109.5 \\
\hline $\mathrm{O} 1 \mathrm{D}-\mathrm{C} 1 \mathrm{D}-\mathrm{C} 2 \mathrm{D}$ & $108.5(5)$ \\
\hline $\mathrm{O} 1 \mathrm{D}-\mathrm{C} 1 \mathrm{D}-\mathrm{C} 8 \mathrm{D}$ & $106.6(5)$ \\
\hline $\mathrm{C} 2 \mathrm{D}-\mathrm{C} 1 \mathrm{D}-\mathrm{C} 8 \mathrm{D}$ & $112.1(5)$ \\
\hline $\mathrm{O} 1 \mathrm{D}-\mathrm{C} 1 \mathrm{D}-\mathrm{C} 14 \mathrm{D}$ & $108.8(5)$ \\
\hline $\mathrm{C} 2 \mathrm{D}-\mathrm{C} 1 \mathrm{D}-\mathrm{C} 14 \mathrm{D}$ & $110.8(5)$ \\
\hline $\mathrm{C} 8 \mathrm{D}-\mathrm{C} 1 \mathrm{D}-\mathrm{C} 14 \mathrm{D}$ & $110.0(5)$ \\
\hline $\mathrm{C} 7 \mathrm{D}-\mathrm{C} 2 \mathrm{D}-\mathrm{C} 3 \mathrm{D}$ & $116.3(11)$ \\
\hline $\mathrm{C} 7 \mathrm{D}-\mathrm{C} 2 \mathrm{D}-\mathrm{C} 1 \mathrm{D}$ & $123.6(10)$ \\
\hline $\mathrm{C} 3 \mathrm{D}-\mathrm{C} 2 \mathrm{D}-\mathrm{C} 1 \mathrm{D}$ & $120.1(6)$ \\
\hline $\mathrm{C} 4 \mathrm{D}-\mathrm{C} 3 \mathrm{D}-\mathrm{C} 2 \mathrm{D}$ & $122.3(9)$ \\
\hline $\mathrm{C} 4 \mathrm{D}-\mathrm{C} 3 \mathrm{D}-\mathrm{H} 3 \mathrm{D}$ & 118.9 \\
\hline $\mathrm{C} 2 \mathrm{D}-\mathrm{C} 3 \mathrm{D}-\mathrm{H} 3 \mathrm{D}$ & 118.9 \\
\hline $\mathrm{C} 3 \mathrm{D}-\mathrm{C} 4 \mathrm{D}-\mathrm{C} 5 \mathrm{D}$ & $121.1(13)$ \\
\hline $\mathrm{C} 3 \mathrm{D}-\mathrm{C} 4 \mathrm{D}-\mathrm{H} 4 \mathrm{D}$ & 119.5 \\
\hline $\mathrm{C} 5 \mathrm{D}-\mathrm{C} 4 \mathrm{D}-\mathrm{H} 4 \mathrm{D}$ & 119.5 \\
\hline $\mathrm{C} 6 \mathrm{D}-\mathrm{C} 5 \mathrm{D}-\mathrm{C} 4 \mathrm{D}$ & 116.0 \\
\hline $\mathrm{C} 6 \mathrm{D}-\mathrm{C} 5 \mathrm{D}-\mathrm{H} 5 \mathrm{D}$ & 122.0 \\
\hline $\mathrm{C} 4 \mathrm{D}-\mathrm{C} 5 \mathrm{D}-\mathrm{H} 5 \mathrm{D}$ & 122.0 \\
\hline $\mathrm{C} 5 \mathrm{D}-\mathrm{C} 6 \mathrm{D}-\mathrm{C} 7 \mathrm{D}$ & $122(2)$ \\
\hline $\mathrm{C} 5 \mathrm{D}-\mathrm{C} 6 \mathrm{D}-\mathrm{H} 6 \mathrm{D}$ & 118.8 \\
\hline
\end{tabular}




\begin{tabular}{|c|c|c|c|}
\hline $\mathrm{C} 5 \mathrm{~B}-\mathrm{C} 6 \mathrm{~B}-\mathrm{C} 7 \mathrm{~B}$ & $120.3(6)$ & $\mathrm{C} 7 \mathrm{D}-\mathrm{C} 6 \mathrm{D}-\mathrm{H} 6 \mathrm{D}$ & 118.8 \\
\hline $\mathrm{C} 5 \mathrm{~B}-\mathrm{C} 6 \mathrm{~B}-\mathrm{H} 6 \mathrm{~B}$ & 119.8 & $\mathrm{C} 2 \mathrm{D}-\mathrm{C} 7 \mathrm{D}-\mathrm{C} 6 \mathrm{D}$ & $121.5(19)$ \\
\hline $\mathrm{C} 7 \mathrm{~B}-\mathrm{C} 6 \mathrm{~B}-\mathrm{H} 6 \mathrm{~B}$ & 119.8 & $\mathrm{C} 2 \mathrm{D}-\mathrm{C} 7 \mathrm{D}-\mathrm{H} 7 \mathrm{D}$ & 119.2 \\
\hline $\mathrm{C} 2 \mathrm{~B}-\mathrm{C} 7 \mathrm{~B}-\mathrm{C} 6 \mathrm{~B}$ & $121.3(4)$ & $\mathrm{C} 6 \mathrm{D}-\mathrm{C} 7 \mathrm{D}-\mathrm{H} 7 \mathrm{D}$ & 119.2 \\
\hline $\mathrm{C} 2 \mathrm{~B}-\mathrm{C} 7 \mathrm{~B}-\mathrm{H} 7 \mathrm{~B}$ & 119.3 & $\mathrm{C} 13 \mathrm{D}-\mathrm{C} 8 \mathrm{D}-\mathrm{C} 9 \mathrm{D}$ & $117.5(9)$ \\
\hline $\mathrm{C} 6 \mathrm{~B}-\mathrm{C} 7 \mathrm{~B}-\mathrm{H} 7 \mathrm{~B}$ & 119.3 & $\mathrm{C} 13 \mathrm{D}-\mathrm{C} 8 \mathrm{D}-\mathrm{C} 1 \mathrm{D}$ & $119.3(6)$ \\
\hline $\mathrm{C} 13 \mathrm{~B}-\mathrm{C} 8 \mathrm{~B}-\mathrm{C} 9 \mathrm{~B}$ & $117.1(3)$ & $\mathrm{C} 9 \mathrm{D}-\mathrm{C} 8 \mathrm{D}-\mathrm{C} 1 \mathrm{D}$ & $123.3(9)$ \\
\hline $\mathrm{C} 13 \mathrm{~B}-\mathrm{C} 8 \mathrm{~B}-\mathrm{C} 1 \mathrm{~B}$ & $122.1(3)$ & $\mathrm{C} 10 \mathrm{D}-\mathrm{C} 9 \mathrm{D}-\mathrm{C} 8 \mathrm{D}$ & $121.6(16)$ \\
\hline $\mathrm{C} 9 \mathrm{~B}-\mathrm{C} 8 \mathrm{~B}-\mathrm{C} 1 \mathrm{~B}$ & $120.7(2)$ & $\mathrm{C} 10 \mathrm{D}-\mathrm{C} 9 \mathrm{D}-\mathrm{H} 9 \mathrm{D}$ & 119.2 \\
\hline $\mathrm{C} 10 \mathrm{~B}-\mathrm{C} 9 \mathrm{~B}-\mathrm{C} 8 \mathrm{~B}$ & $121.4(5)$ & $\mathrm{C} 8 \mathrm{D}-\mathrm{C} 9 \mathrm{D}-\mathrm{H} 9 \mathrm{D}$ & 119.2 \\
\hline $\mathrm{C} 10 \mathrm{~B}-\mathrm{C} 9 \mathrm{~B}-\mathrm{H} 9 \mathrm{~B}$ & 119.3 & $\mathrm{C} 9 \mathrm{D}-\mathrm{C} 10 \mathrm{D}-\mathrm{C} 11 \mathrm{D}$ & $117.4(16)$ \\
\hline $\mathrm{C} 8 \mathrm{~B}-\mathrm{C} 9 \mathrm{~B}-\mathrm{H} 9 \mathrm{~B}$ & 119.3 & $\mathrm{C} 9 \mathrm{D}-\mathrm{C} 10 \mathrm{D}-\mathrm{H} 10 \mathrm{D}$ & 121.3 \\
\hline $\mathrm{C} 9 \mathrm{~B}-\mathrm{C} 10 \mathrm{~B}-\mathrm{C} 11 \mathrm{~B}$ & $121.5(8)$ & $\mathrm{C} 11 \mathrm{D}-\mathrm{C} 10 \mathrm{D}-\mathrm{H} 10 \mathrm{D}$ & 121.3 \\
\hline $\mathrm{C} 9 \mathrm{~B}-\mathrm{C} 10 \mathrm{~B}-\mathrm{H} 10 \mathrm{~B}$ & 119.3 & $\mathrm{C} 10 \mathrm{D}-\mathrm{C} 11 \mathrm{D}-\mathrm{C} 12 \mathrm{D}$ & $124.7(14)$ \\
\hline $\mathrm{C} 11 \mathrm{~B}-\mathrm{C} 10 \mathrm{~B}-\mathrm{H} 10 \mathrm{~B}$ & 119.3 & $\mathrm{C} 10 \mathrm{D}-\mathrm{C} 11 \mathrm{D}-\mathrm{H} 11 \mathrm{D}$ & 117.6 \\
\hline $\mathrm{C} 10 \mathrm{~B}-\mathrm{C} 11 \mathrm{~B}-\mathrm{C} 12 \mathrm{~B}$ & $120.1(8)$ & $\mathrm{C} 12 \mathrm{D}-\mathrm{C} 11 \mathrm{D}-\mathrm{H} 11 \mathrm{D}$ & 117.6 \\
\hline $\mathrm{C} 10 \mathrm{~B}-\mathrm{C} 11 \mathrm{~B}-\mathrm{H} 11 \mathrm{~B}$ & 119.9 & $\mathrm{C} 13 \mathrm{D}-\mathrm{C} 12 \mathrm{D}-\mathrm{C} 11 \mathrm{D}$ & $114.4(12)$ \\
\hline $\mathrm{C} 12 \mathrm{~B}-\mathrm{C} 11 \mathrm{~B}-\mathrm{H} 11 \mathrm{~B}$ & 119.9 & $\mathrm{C} 13 \mathrm{D}-\mathrm{C} 12 \mathrm{D}-\mathrm{H} 12 \mathrm{D}$ & 122.8 \\
\hline $\mathrm{C} 11 \mathrm{~B}-\mathrm{C} 12 \mathrm{~B}-\mathrm{C} 13 \mathrm{~B}$ & $117.4(8)$ & $\mathrm{C} 11 \mathrm{D}-\mathrm{C} 12 \mathrm{D}-\mathrm{H} 12 \mathrm{D}$ & 122.8 \\
\hline $\mathrm{C} 11 \mathrm{~B}-\mathrm{C} 12 \mathrm{~B}-\mathrm{H} 12 \mathrm{~B}$ & 121.3 & $\mathrm{C} 8 \mathrm{D}-\mathrm{C} 13 \mathrm{D}-\mathrm{C} 12 \mathrm{D}$ & $123.6(8)$ \\
\hline $\mathrm{C} 13 \mathrm{~B}-\mathrm{C} 12 \mathrm{~B}-\mathrm{H} 12 \mathrm{~B}$ & 121.3 & $\mathrm{C} 8 \mathrm{D}-\mathrm{C} 13 \mathrm{D}-\mathrm{H} 13 \mathrm{D}$ & 118.2 \\
\hline $\mathrm{C} 8 \mathrm{~B}-\mathrm{C} 13 \mathrm{~B}-\mathrm{C} 12 \mathrm{~B}$ & $122.5(5)$ & $\mathrm{C} 12 \mathrm{D}-\mathrm{C} 13 \mathrm{D}-\mathrm{H} 13 \mathrm{D}$ & 118.2 \\
\hline $\mathrm{C} 8 \mathrm{~B}-\mathrm{C} 13 \mathrm{~B}-\mathrm{H} 13 \mathrm{~B}$ & 118.8 & $\mathrm{C} 15 \mathrm{D}-\mathrm{C} 14 \mathrm{D}-\mathrm{C} 19 \mathrm{D}$ & $118.6(9)$ \\
\hline $\mathrm{C} 12 \mathrm{~B}-\mathrm{C} 13 \mathrm{~B}-\mathrm{H} 13 \mathrm{~B}$ & 118.8 & $\mathrm{C} 15 \mathrm{D}-\mathrm{C} 14 \mathrm{D}-\mathrm{C} 1 \mathrm{D}$ & $119.7(6)$ \\
\hline $\mathrm{C} 15 \mathrm{~B}-\mathrm{C} 14 \mathrm{~B}-\mathrm{C} 19 \mathrm{~B}$ & $117.5(3)$ & $\mathrm{C} 19 \mathrm{D}-\mathrm{C} 14 \mathrm{D}-\mathrm{C} 1 \mathrm{D}$ & $121.7(9)$ \\
\hline $\mathrm{C} 15 \mathrm{~B}-\mathrm{C} 14 \mathrm{~B}-\mathrm{C} 1 \mathrm{~B}$ & $119.1(2)$ & $\mathrm{C} 14 \mathrm{D}-\mathrm{C} 15 \mathrm{D}-\mathrm{C} 16 \mathrm{D}$ & $120.5(11)$ \\
\hline $\mathrm{C} 19 \mathrm{~B}-\mathrm{C} 14 \mathrm{~B}-\mathrm{C} 1 \mathrm{~B}$ & $123.4(3)$ & $\mathrm{C} 14 \mathrm{D}-\mathrm{C} 15 \mathrm{D}-\mathrm{H} 15 \mathrm{D}$ & 119.7 \\
\hline $\mathrm{C} 16 \mathrm{~B}-\mathrm{C} 15 \mathrm{~B}-\mathrm{C} 14 \mathrm{~B}$ & $121.1(3)$ & $\mathrm{C} 16 \mathrm{D}-\mathrm{C} 15 \mathrm{D}-\mathrm{H} 15 \mathrm{D}$ & 119.7 \\
\hline $\mathrm{C} 16 \mathrm{~B}-\mathrm{C} 15 \mathrm{~B}-\mathrm{H} 15 \mathrm{~B}$ & 119.4 & $\mathrm{C} 17 \mathrm{D}-\mathrm{C} 16 \mathrm{D}-\mathrm{C} 15 \mathrm{D}$ & $121.1(16)$ \\
\hline $\mathrm{C} 14 \mathrm{~B}-\mathrm{C} 15 \mathrm{~B}-\mathrm{H} 15 \mathrm{~B}$ & 119.4 & $\mathrm{C} 17 \mathrm{D}-\mathrm{C} 16 \mathrm{D}-\mathrm{H} 16 \mathrm{D}$ & 119.5 \\
\hline $\mathrm{C} 15 \mathrm{~B}-\mathrm{C} 16 \mathrm{~B}-\mathrm{C} 17 \mathrm{~B}$ & $121.6(4)$ & $\mathrm{C} 15 \mathrm{D}-\mathrm{C} 16 \mathrm{D}-\mathrm{H} 16 \mathrm{D}$ & 119.5 \\
\hline $\mathrm{C} 15 \mathrm{~B}-\mathrm{C} 16 \mathrm{~B}-\mathrm{H} 16 \mathrm{~B}$ & 119.2 & $\mathrm{C} 18 \mathrm{D}-\mathrm{C} 17 \mathrm{D}-\mathrm{C} 16 \mathrm{D}$ & $118.2(16)$ \\
\hline $\mathrm{C} 17 \mathrm{~B}-\mathrm{C} 16 \mathrm{~B}-\mathrm{H} 16 \mathrm{~B}$ & 119.2 & $\mathrm{C} 18 \mathrm{D}-\mathrm{C} 17 \mathrm{D}-\mathrm{H} 17 \mathrm{D}$ & 120.9 \\
\hline $\mathrm{C} 18 \mathrm{~B}-\mathrm{C} 17 \mathrm{~B}-\mathrm{C} 16 \mathrm{~B}$ & $116.9(4)$ & $\mathrm{C} 16 \mathrm{D}-\mathrm{C} 17 \mathrm{D}-\mathrm{H} 17 \mathrm{D}$ & 120.9 \\
\hline $\mathrm{C} 18 \mathrm{~B}-\mathrm{C} 17 \mathrm{~B}-\mathrm{H} 17 \mathrm{~B}$ & 121.5 & $\mathrm{C} 17 \mathrm{D}-\mathrm{C} 18 \mathrm{D}-\mathrm{C} 19 \mathrm{D}$ & $120.2(16)$ \\
\hline $\mathrm{C} 16 \mathrm{~B}-\mathrm{C} 17 \mathrm{~B}-\mathrm{H} 17 \mathrm{~B}$ & 121.5 & $\mathrm{C} 17 \mathrm{D}-\mathrm{C} 18 \mathrm{D}-\mathrm{H} 18 \mathrm{D}$ & 119.9 \\
\hline $\mathrm{C} 19 \mathrm{~B}-\mathrm{C} 18 \mathrm{~B}-\mathrm{C} 17 \mathrm{~B}$ & $121.5(4)$ & $\mathrm{C} 19 \mathrm{D}-\mathrm{C} 18 \mathrm{D}-\mathrm{H} 18 \mathrm{D}$ & 119.9 \\
\hline $\mathrm{C} 19 \mathrm{~B}-\mathrm{C} 18 \mathrm{~B}-\mathrm{H} 18 \mathrm{~B}$ & 119.3 & $\mathrm{C} 14 \mathrm{D}-\mathrm{C} 19 \mathrm{D}-\mathrm{C} 18 \mathrm{D}$ & $121.2(16)$ \\
\hline $\mathrm{C} 17 \mathrm{~B}-\mathrm{C} 18 \mathrm{~B}-\mathrm{H} 18 \mathrm{~B}$ & 119.3 & $\mathrm{C} 14 \mathrm{D}-\mathrm{C} 19 \mathrm{D}-\mathrm{H} 19 \mathrm{D}$ & 119.4 \\
\hline $\mathrm{C} 18 \mathrm{~B}-\mathrm{C} 19 \mathrm{~B}-\mathrm{C} 14 \mathrm{~B}$ & $121.2(5)$ & $\mathrm{C} 18 \mathrm{D}-\mathrm{C} 19 \mathrm{D}-\mathrm{H} 19 \mathrm{D}$ & 119.4 \\
\hline $\mathrm{C} 18 \mathrm{~B}-\mathrm{C} 19 \mathrm{~B}-\mathrm{H} 19 \mathrm{~B}$ & 119.4 & & \\
\hline $\mathrm{O} 1 \mathrm{~A}-\mathrm{C} 1 \mathrm{~A}-\mathrm{C} 2 \mathrm{~A}-\mathrm{C} 3 \mathrm{~A}$ & $35.5(2)$ & $\mathrm{O} 1 \mathrm{C}-\mathrm{C} 1 \mathrm{C}-\mathrm{C} 2 \mathrm{C}-\mathrm{C} 7 \mathrm{C}$ & $31.0(9)$ \\
\hline $\mathrm{C} 2 \mathrm{~A}^{\mathrm{ii}}-\mathrm{C} 1 \mathrm{~A}-\mathrm{C} 2 \mathrm{~A}-\mathrm{C} 3 \mathrm{~A}$ & $-82.7(3)$ & $\mathrm{C} 2 \mathrm{C}^{\mathrm{ii}}-\mathrm{C} 1 \mathrm{C}-\mathrm{C} 2 \mathrm{C}-\mathrm{C} 7 \mathrm{C}$ & $150.7(9)$ \\
\hline $\mathrm{C} 2 \mathrm{~A}^{\mathrm{L}}-\mathrm{C} 1 \mathrm{~A}-\mathrm{C} 2 \mathrm{~A}-\mathrm{C} 3 \mathrm{~A}$ & $153.6(2)$ & $\mathrm{C} 2 \mathrm{C}-\mathrm{C} 1 \mathrm{C}-\mathrm{C} 2 \mathrm{C}-\mathrm{C} 7 \mathrm{C}$ & $-88.8(12)$ \\
\hline $\mathrm{O} 1 \mathrm{~A}-\mathrm{C} 1 \mathrm{~A}-\mathrm{C} 2 \mathrm{~A}-\mathrm{C} 7 \mathrm{~A}$ & $-146.7(4)$ & $\mathrm{O} 1 \mathrm{C}-\mathrm{C} 1 \mathrm{C}-\mathrm{C} 2 \mathrm{C}-\mathrm{C} 3 \mathrm{C}$ & $-145.6(9)$ \\
\hline $\mathrm{C} 2 \mathrm{~A}^{\mathrm{ii}}-\mathrm{C} 1 \mathrm{~A}-\mathrm{C} 2 \mathrm{~A}-\mathrm{C} 7 \mathrm{~A}$ & $95.1(4)$ & $\mathrm{C} 2 \mathrm{C}^{\mathrm{ii}}-\mathrm{C} 1 \mathrm{C}-\mathrm{C} 2 \mathrm{C}-\mathrm{C} 3 \mathrm{C}$ & $-25.8(12)$ \\
\hline $\mathrm{C} 2 \mathrm{~A}^{ }-\mathrm{C} 1 \mathrm{~A}-\mathrm{C} 2 \mathrm{~A}-\mathrm{C} 7 \mathrm{~A}$ & $-28.6(5)$ & $\mathrm{C} 2 \mathrm{C}^{\mathrm{i}}-\mathrm{C} 1 \mathrm{C}-\mathrm{C} 2 \mathrm{C}-\mathrm{C} 3 \mathrm{C}$ & $94.6(9)$ \\
\hline
\end{tabular}




\begin{tabular}{|c|c|}
\hline $\mathrm{C} 7 \mathrm{~A}-\mathrm{C} 2 \mathrm{~A}-\mathrm{C} 3 \mathrm{~A}-\mathrm{C} 4 \mathrm{~A}$ & $-1.6(6)$ \\
\hline $\mathrm{C} 1 \mathrm{~A}-\mathrm{C} 2 \mathrm{~A}-\mathrm{C} 3 \mathrm{~A}-\mathrm{C} 4 \mathrm{~A}$ & $176.3(4)$ \\
\hline $\mathrm{C} 2 \mathrm{~A}-\mathrm{C} 3 \mathrm{~A}-\mathrm{C} 4 \mathrm{~A}-\mathrm{C} 5 \mathrm{~A}$ & $-0.1(9)$ \\
\hline $\mathrm{C} 3 \mathrm{~A}-\mathrm{C} 4 \mathrm{~A}-\mathrm{C} 5 \mathrm{~A}-\mathrm{C} 6 \mathrm{~A}$ & $1.9(12)$ \\
\hline $\mathrm{C} 4 \mathrm{~A}-\mathrm{C} 5 \mathrm{~A}-\mathrm{C} 6 \mathrm{~A}-\mathrm{C} 7 \mathrm{~A}$ & $-1.9(12)$ \\
\hline $\mathrm{C} 5 \mathrm{~A}-\mathrm{C} 6 \mathrm{~A}-\mathrm{C} 7 \mathrm{~A}-\mathrm{C} 2 \mathrm{~A}$ & $0.0(10)$ \\
\hline $\mathrm{C} 3 \mathrm{~A}-\mathrm{C} 2 \mathrm{~A}-\mathrm{C} 7 \mathrm{~A}-\mathrm{C} 6 \mathrm{~A}$ & $1.7(8)$ \\
\hline $\mathrm{C} 1 \mathrm{~A}-\mathrm{C} 2 \mathrm{~A}-\mathrm{C} 7 \mathrm{~A}-\mathrm{C} 6 \mathrm{~A}$ & $-176.2(5)$ \\
\hline $\mathrm{O} 1 \mathrm{~B}-\mathrm{C} 1 \mathrm{~B}-\mathrm{C} 2 \mathrm{~B}-\mathrm{C} 7 \mathrm{~B}$ & $-137.2(3)$ \\
\hline $\mathrm{C} 8 \mathrm{~B}-\mathrm{C} 1 \mathrm{~B}-\mathrm{C} 2 \mathrm{~B}-\mathrm{C} 7 \mathrm{~B}$ & $104.2(3)$ \\
\hline $\mathrm{C} 14 \mathrm{~B}-\mathrm{C} 1 \mathrm{~B}-\mathrm{C} 2 \mathrm{~B}-\mathrm{C} 7 \mathrm{~B}$ & $-20.2(4)$ \\
\hline $\mathrm{O} 1 \mathrm{~B}-\mathrm{C} 1 \mathrm{~B}-\mathrm{C} 2 \mathrm{~B}-\mathrm{C} 3 \mathrm{~B}$ & $44.6(3)$ \\
\hline $\mathrm{C} 8 \mathrm{~B}-\mathrm{C} 1 \mathrm{~B}-\mathrm{C} 2 \mathrm{~B}-\mathrm{C} 3 \mathrm{~B}$ & $-73.9(3)$ \\
\hline $\mathrm{C} 14 \mathrm{~B}-\mathrm{C} 1 \mathrm{~B}-\mathrm{C} 2 \mathrm{~B}-\mathrm{C} 3 \mathrm{~B}$ & $161.7(3)$ \\
\hline $\mathrm{C} 7 \mathrm{~B}-\mathrm{C} 2 \mathrm{~B}-\mathrm{C} 3 \mathrm{~B}-\mathrm{C} 4 \mathrm{~B}$ & $1.8(6)$ \\
\hline $\mathrm{C} 1 \mathrm{~B}-\mathrm{C} 2 \mathrm{~B}-\mathrm{C} 3 \mathrm{~B}-\mathrm{C} 4 \mathrm{~B}$ & $-180.0(5)$ \\
\hline $\mathrm{C} 2 \mathrm{~B}-\mathrm{C} 3 \mathrm{~B}-\mathrm{C} 4 \mathrm{~B}-\mathrm{C} 5 \mathrm{~B}$ & $-2.0(11)$ \\
\hline $\mathrm{C} 3 \mathrm{~B}-\mathrm{C} 4 \mathrm{~B}-\mathrm{C} 5 \mathrm{~B}-\mathrm{C} 6 \mathrm{~B}$ & $1.3(13)$ \\
\hline $\mathrm{C} 4 \mathrm{~B}-\mathrm{C} 5 \mathrm{~B}-\mathrm{C} 6 \mathrm{~B}-\mathrm{C} 7 \mathrm{~B}$ & $-0.6(12)$ \\
\hline $\mathrm{C} 3 \mathrm{~B}-\mathrm{C} 2 \mathrm{~B}-\mathrm{C} 7 \mathrm{~B}-\mathrm{C} 6 \mathrm{~B}$ & $-1.2(6)$ \\
\hline $\mathrm{C} 1 \mathrm{~B}-\mathrm{C} 2 \mathrm{~B}-\mathrm{C} 7 \mathrm{~B}-\mathrm{C} 6 \mathrm{~B}$ & $-179.3(4)$ \\
\hline $\mathrm{C} 5 \mathrm{~B}-\mathrm{C} 6 \mathrm{~B}-\mathrm{C} 7 \mathrm{~B}-\mathrm{C} 2 \mathrm{~B}$ & $0.6(9)$ \\
\hline $\mathrm{O} 1 \mathrm{~B}-\mathrm{C} 1 \mathrm{~B}-\mathrm{C} 8 \mathrm{~B}-\mathrm{C} 13 \mathrm{~B}$ & $-147.8(3)$ \\
\hline $\mathrm{C} 2 \mathrm{~B}-\mathrm{C} 1 \mathrm{~B}-\mathrm{C} 8 \mathrm{~B}-\mathrm{C} 13 \mathrm{~B}$ & $-30.0(3)$ \\
\hline $\mathrm{C} 14 \mathrm{~B}-\mathrm{C} 1 \mathrm{~B}-\mathrm{C} 8 \mathrm{~B}-\mathrm{C} 13 \mathrm{~B}$ & $94.2(3)$ \\
\hline $\mathrm{O} 1 \mathrm{~B}-\mathrm{C} 1 \mathrm{~B}-\mathrm{C} 8 \mathrm{~B}-\mathrm{C} 9 \mathrm{~B}$ & $35.3(3)$ \\
\hline $\mathrm{C} 2 \mathrm{~B}-\mathrm{C} 1 \mathrm{~B}-\mathrm{C} 8 \mathrm{~B}-\mathrm{C} 9 \mathrm{~B}$ & $153.1(2)$ \\
\hline $\mathrm{C} 14 \mathrm{~B}-\mathrm{C} 1 \mathrm{~B}-\mathrm{C} 8 \mathrm{~B}-\mathrm{C} 9 \mathrm{~B}$ & $-82.8(3)$ \\
\hline $\mathrm{C} 13 \mathrm{~B}-\mathrm{C} 8 \mathrm{~B}-\mathrm{C} 9 \mathrm{~B}-\mathrm{C} 10 \mathrm{~B}$ & $1.5(5)$ \\
\hline $\mathrm{C} 1 \mathrm{~B}-\mathrm{C} 8 \mathrm{~B}-\mathrm{C} 9 \mathrm{~B}-\mathrm{C} 10 \mathrm{~B}$ & $178.5(4)$ \\
\hline $\mathrm{C} 8 \mathrm{~B}-\mathrm{C} 9 \mathrm{~B}-\mathrm{C} 10 \mathrm{~B}-\mathrm{C} 11 \mathrm{~B}$ & $-3.4(9)$ \\
\hline $\mathrm{C} 9 \mathrm{~B}-\mathrm{C} 10 \mathrm{~B}-\mathrm{C} 11 \mathrm{~B}-\mathrm{C} 12 \mathrm{~B}$ & $3.7(12)$ \\
\hline $\mathrm{C} 10 \mathrm{~B}-\mathrm{C} 11 \mathrm{~B}-\mathrm{C} 12 \mathrm{~B}-\mathrm{C} 13 \mathrm{~B}$ & $-2.0(12)$ \\
\hline $\mathrm{C} 9 \mathrm{~B}-\mathrm{C} 8 \mathrm{~B}-\mathrm{C} 13 \mathrm{~B}-\mathrm{C} 12 \mathrm{~B}$ & $0.1(7)$ \\
\hline $\mathrm{C} 1 \mathrm{~B}-\mathrm{C} 8 \mathrm{~B}-\mathrm{C} 13 \mathrm{~B}-\mathrm{C} 12 \mathrm{~B}$ & $-176.9(5)$ \\
\hline $\mathrm{C} 11 \mathrm{~B}-\mathrm{C} 12 \mathrm{~B}-\mathrm{C} 13 \mathrm{~B}-\mathrm{C} 8 \mathrm{~B}$ & $0.2(11)$ \\
\hline $\mathrm{O} 1 \mathrm{~B}-\mathrm{C} 1 \mathrm{~B}-\mathrm{C} 14 \mathrm{~B}-\mathrm{C} 15 \mathrm{~B}$ & $42.8(4)$ \\
\hline $\mathrm{C} 8 \mathrm{~B}-\mathrm{C} 1 \mathrm{~B}-\mathrm{C} 14 \mathrm{~B}-\mathrm{C} 15 \mathrm{~B}$ & $161.5(3)$ \\
\hline $\mathrm{C} 2 \mathrm{~B}-\mathrm{C} 1 \mathrm{~B}-\mathrm{C} 14 \mathrm{~B}-\mathrm{C} 15 \mathrm{~B}$ & $-74.1(3)$ \\
\hline $\mathrm{O} 1 \mathrm{~B}-\mathrm{C} 1 \mathrm{~B}-\mathrm{C} 14 \mathrm{~B}-\mathrm{C} 19 \mathrm{~B}$ & $-139.0(4)$ \\
\hline $\mathrm{C} 8 \mathrm{~B}-\mathrm{C} 1 \mathrm{~B}-\mathrm{C} 14 \mathrm{~B}-\mathrm{C} 19 \mathrm{~B}$ & $-20.3(5)$ \\
\hline $\mathrm{C} 2 \mathrm{~B}-\mathrm{C} 1 \mathrm{~B}-\mathrm{C} 14 \mathrm{~B}-\mathrm{C} 19 \mathrm{~B}$ & $104.1(5)$ \\
\hline $\mathrm{C} 19 \mathrm{~B}-\mathrm{C} 14 \mathrm{~B}-\mathrm{C} 15 \mathrm{~B}-\mathrm{C} 16 \mathrm{~B}$ & $-1.9(6)$ \\
\hline $\mathrm{C} 1 \mathrm{~B}-\mathrm{C} 14 \mathrm{~B}-\mathrm{C} 15 \mathrm{~B}-\mathrm{C} 16 \mathrm{~B}$ & $176.4(4)$ \\
\hline $\mathrm{C} 14 \mathrm{~B}-\mathrm{C} 15 \mathrm{~B}-\mathrm{C} 16 \mathrm{~B}-\mathrm{C} 17 \mathrm{~B}$ & $0.3(8)$ \\
\hline $\mathrm{C} 15 \mathrm{~B}-\mathrm{C} 16 \mathrm{~B}-\mathrm{C} 17 \mathrm{~B}-\mathrm{C} 18 \mathrm{~B}$ & $0.9(8)$ \\
\hline $\mathrm{C} 16 \mathrm{~B}-\mathrm{C} 17 \mathrm{~B}-\mathrm{C} 18 \mathrm{~B}-\mathrm{C} 19 \mathrm{~B}$ & $-0.4(7)$ \\
\hline $\mathrm{C} 17 \mathrm{~B}-\mathrm{C} 18 \mathrm{~B}-\mathrm{C} 19 \mathrm{~B}-\mathrm{C} 14 \mathrm{~B}$ & $-1.4(8)$ \\
\hline
\end{tabular}

$\begin{array}{ll}\text { C7C-C2C-C3C-C4C } & -0.7(14) \\ \text { C1C-C2C-C3C-C4C } & 175.9(10) \\ \text { C2C-C3C-C4C-C5C } & 3.2(17) \\ \text { C3C-C4C-C5C-C6C } & -5(3) \\ \text { C4C-C5C-C6C-C7C } & 5(4) \\ \text { C5C-C6C-C7C-C2C } & -2(4) \\ \text { C3C-C2C-C7C-C6C } & 0(2) \\ \text { C1C-C2C-C7C-C6C } & -176.6(19) \\ \text { O1D-C1D-C2D-C7D } & -126.3(16) \\ \text { C8D-C1D-C2D-C7D } & 116.3(17) \\ \text { C14D-C1D-C2D-C7D } & -7.0(17) \\ \text { O1D-C1D-C2D-C3D } & 51.4(8) \\ \text { C8D-C1D-C2D-C3D } & -66.0(8) \\ \text { C14D-C1D-C2D-C3D } & 170.8(6) \\ \text { C7D-C2D-C3D-C4D } & -6.0(17) \\ \text { C1D-C2D-C3D-C4D } & 176.1(8) \\ \text { C2D-C3D-C4D-C5D } & 1.0(17) \\ \text { C3D-C4D-C5D-C6D } & 2(3) \\ \text { C4D-C5D-C6D-C7D } & 1(4) \\ \text { C3D-C2D-C7D-C6D } & 8(3) \\ \text { C1D-C2D-C7D-C6D } & -173.9(18) \\ \text { C5D-C6D-C7D-C2D } & -6(4) \\ \text { O1D-C1D-C8D-C13D } & 40.9(9) \\ \text { C2D-C1D-C8D-C13D } & 159.4(7) \\ \text { C14D-C1D-C8D-C13D } & -76.9(8) \\ \text { O1D-C1D-C8D-C9D } & -140.6(9) \\ \text { C2D-C1D-C8D-C9D } & -22.1(10) \\ \text { C14D-C1D-C8D-C9D } & 101.6(9) \\ \text { C13D-C8D-C9D-C10D } & 1.9(14) \\ \text { C1D-C8D-C9D-C10D } & -176.6(9) \\ \text { C8D-C9D-C10D-C11D } & -3.5(16) \\ \text { C9D-C10D-C11D-C12D } & 8(2) \\ \text { C10D-C11D-C12D-C13D } & -11(3) \\ \text { C9D-C8D-C13D-C12D } & -4.9(16) \\ \text { C1D-C8D-C13D-C12D } & 173.7(10) \\ \text { C11D-C12D-C13D-C8D } & 9(2) \\ \text { O1D-C1D-C14D-C15D } & 29.5(8) \\ \text { C2D-C1D-C14D-C15D } & -89.7(7) \\ \text { C8D-C1D-C14D-C15D } & 145.9(6) \\ \text { O1D-C1D-C14D-C19D } & -149.5(12) \\ \text { C2D-C1D-C14D-C19D } & 91.4(13) \\ \text { C8D-C1D-C14D-C19D } & -33.0(13) \\ \text { C19D-C14D-C15D-C16D } & -1.7(14) \\ \text { C1D-C14D-C15D-C16D } & 179.4(10) \\ \text { C14D-C15D-C16D-C17D } & -2.7(19) \\ \text { C15D-C16D-C17D-C18D } & 5(3) \\ \text { C16D-C17D-C18D-C19D } & -3(3) \\ \text { C15D-C14D-C19D-C18D } & 4(3) \\ & \end{array}$




\begin{tabular}{llll}
$\mathrm{C} 15 \mathrm{~B}-\mathrm{C} 14 \mathrm{~B}-\mathrm{C} 19 \mathrm{~B}-\mathrm{C} 18 \mathrm{~B}$ & $2.5(8)$ & $\mathrm{C} 1 \mathrm{D}-\mathrm{C} 14 \mathrm{D}-\mathrm{C} 19 \mathrm{D}-\mathrm{C} 18 \mathrm{D}$ & $-177.3(16)$ \\
$\mathrm{C} 1 \mathrm{~B}-\mathrm{C} 14 \mathrm{~B}-\mathrm{C} 19 \mathrm{~B}-\mathrm{C} 18 \mathrm{~B}$ & $-175.8(4)$ & $\mathrm{C} 17 \mathrm{D}-\mathrm{C} 18 \mathrm{D}-\mathrm{C} 19 \mathrm{D}-\mathrm{C} 14 \mathrm{D}$ & $-2(3)$ \\
\hline
\end{tabular}

Symmetry codes: (i) $-y+1, x-y, z$; (ii) $-x+y+1,-x+1, z$.

Hydrogen-bond geometry $\left(\AA,{ }^{\circ}\right)$

\begin{tabular}{lllll}
\hline$D-\mathrm{H} \cdots A$ & $D-\mathrm{H}$ & $\mathrm{H} \cdots A$ & $D \cdots A$ & $D-\mathrm{H} \cdots A$ \\
\hline $\mathrm{O} 1 A-\mathrm{H} 1 A \cdots \mathrm{O} 1 B$ & 0.85 & 2.33 & $2.869(3)$ & 121 \\
$\mathrm{O} 1 B-\mathrm{H} 1 B A \cdots \mathrm{O} 1 A$ & 0.84 & 2.27 & $2.869(3)$ & 129 \\
$\mathrm{O} 1 B-\mathrm{H} 1 B B \cdots \mathrm{O} 1 B^{\mathrm{i}}$ & 0.82 & 2.21 & $2.869(3)$ & 138 \\
$\mathrm{O} 1 B-\mathrm{H} 1 B C \cdots \mathrm{O} 1 B^{\mathrm{ii}}$ & 0.86 & 2.24 & $2.869(3)$ & 130 \\
$\mathrm{O} 1 C-\mathrm{H} 1 C \cdots \mathrm{O} 1 D$ & 0.85 & 2.22 & $2.856(7)$ & 131 \\
$\mathrm{O} 1 D-\mathrm{H} 1 D A \cdots \mathrm{O} 1 C$ & 0.84 & 2.24 & $2.856(7)$ & 131 \\
$\mathrm{O} 1 D-\mathrm{H} 1 D B \cdots \mathrm{O} 1 D^{\mathrm{ii}}$ & 0.82 & 2.38 & $2.951(8)$ & 127 \\
$\mathrm{O} 1 D-\mathrm{H} 1 D C \cdots \mathrm{O} 1 D^{\mathrm{i}}$ & 0.85 & 2.33 & $2.951(8)$ & 131
\end{tabular}

Symmetry codes: (i) $-y+1, x-y, z$; (ii) $-x+y+1,-x+1, z$.

Triphenylmethanol (295K_data)

Crystal data

$\mathrm{C}_{19} \mathrm{H}_{16} \mathrm{O}$

$M_{r}=260.32$

Trigonal, $R \overline{3}: H$

$a=19.3309$ (8) $\AA$

$c=26.8542(11) \AA$

$V=8690.5(8) \AA^{3}$

$Z=24$

$F(000)=3312$

$D_{\mathrm{x}}=1.194 \mathrm{Mg} \mathrm{m}^{-3}$

\section{Data collection}

Stoe Stadivari diffractometer

Radiation source: Sealed X-ray tube, Axo Astixf Microfocus source

Graded multilayer mirror monochromator

Detector resolution: 5.81 pixels $\mathrm{mm}^{-1}$

$\omega$ scans

Absorption correction: multi-scan

(X-AREA; Stoe \& Cie, 2018)

\section{Refinement}

Refinement on $F^{2}$

Least-squares matrix: full

$R\left[F^{2}>2 \sigma\left(F^{2}\right)\right]=0.063$

$w R\left(F^{2}\right)=0.242$

$S=0.99$

4523 reflections

483 parameters

279 restraints

0 constraints

Primary atom site location: dual
Melting point: $433 \mathrm{~K}$

$\mathrm{Ag} K \alpha$ radiation, $\lambda=0.56083 \AA$

Cell parameters from 22044 reflections

$\theta=2.3-23.6^{\circ}$

$\mu=0.05 \mathrm{~mm}^{-1}$

$T=295 \mathrm{~K}$

Prism, colourless

$0.38 \times 0.33 \times 0.33 \mathrm{~mm}$

$T_{\min }=0.558, T_{\max }=1.000$

72080 measured reflections

4523 independent reflections

1656 reflections with $I>2 \sigma(I)$

$R_{\text {int }}=0.108$

$\theta_{\text {max }}=21.5^{\circ}, \theta_{\min }=2.3^{\circ}$

$h=-25 \rightarrow 25$

$k=-25 \rightarrow 25$

$l=-35 \rightarrow 35$

Secondary atom site location: difference Fourier map

Hydrogen site location: mixed

$\mathrm{H}$-atom parameters constrained

$w=1 /\left[\sigma^{2}\left(F_{\mathrm{o}}{ }^{2}\right)+(0.1204 P)^{2}\right]$

where $P=\left(F_{\mathrm{o}}^{2}+2 F_{\mathrm{c}}^{2}\right) / 3$

$(\Delta / \sigma)_{\max }<0.001$

$\Delta \rho_{\max }=0.11$ e $\AA^{-3}$

$\Delta \rho_{\min }=-0.14$ e $\AA^{-3}$ 
Fractional atomic coordinates and isotropic or equivalent isotropic displacement parameters $\left(\AA^{2}\right)$

\begin{tabular}{|c|c|c|c|c|c|}
\hline & $x$ & $y$ & $z$ & $U_{\text {iso }} * / U_{\text {eq }}$ & Occ. $(<1)$ \\
\hline O1A & 0.666667 & 0.333333 & $0.51270(12)$ & 0.0914 (13) & $0.761(3)$ \\
\hline H1A & 0.694157 & 0.379899 & 0.524947 & $0.137 *$ & $0.2537(10)$ \\
\hline $\mathrm{C} 1 \mathrm{~A}$ & 0.666667 & 0.333333 & $0.45924(16)$ & $0.0615(13)$ & $0.761(3)$ \\
\hline $\mathrm{C} 2 \mathrm{~A}$ & $0.58276(19)$ & 0.31029 (19) & $0.44168(11)$ & $0.0616(8)$ & $0.761(3)$ \\
\hline $\mathrm{C} 3 \mathrm{~A}$ & $0.5387(2)$ & $0.3366(3)$ & $0.46772(14)$ & $0.0858(11)$ & $0.761(3)$ \\
\hline $\mathrm{H} 3 \mathrm{~A}$ & 0.559553 & 0.366478 & 0.496593 & $0.103 *$ & $0.761(3)$ \\
\hline $\mathrm{C} 4 \mathrm{~A}$ & $0.4638(6)$ & $0.3188(7)$ & $0.4513(4)$ & $0.116(3)$ & $0.761(3)$ \\
\hline $\mathrm{H} 4 \mathrm{~A}$ & 0.434020 & 0.336006 & 0.469166 & $0.140 *$ & $0.761(3)$ \\
\hline C5A & $0.4335(5)$ & $0.2751(6)$ & $0.4078(4)$ & $0.114(4)$ & $0.761(3)$ \\
\hline $\mathrm{H} 5 \mathrm{~A}$ & 0.383723 & 0.264142 & 0.396160 & $0.137 *$ & $0.761(3)$ \\
\hline C6A & $0.4756(5)$ & $0.2482(6)$ & 0.3821 & $0.103(3)$ & $0.761(3)$ \\
\hline H6A & 0.454732 & 0.218150 & 0.353258 & $0.124 *$ & $0.761(3)$ \\
\hline C7A & $0.5496(5)$ & $0.2659(5)$ & $0.3991(2)$ & $0.0808(16)$ & $0.761(3)$ \\
\hline H7A & 0.578390 & 0.247372 & 0.381366 & $0.097 *$ & $0.761(3)$ \\
\hline O1B & $0.76514(12)$ & $0.36549(16)$ & $0.60145(9)$ & $0.0946(8)$ & $0.761(3)$ \\
\hline H1BA & 0.763528 & 0.369221 & 0.571207 & $0.142 *$ & $0.2537(10)$ \\
\hline H1BB & 0.746050 & 0.388243 & 0.617060 & $0.142 *$ & $0.2537(10)$ \\
\hline $\mathrm{H} 1 \mathrm{BC}$ & 0.737008 & 0.315795 & 0.608270 & $0.142 *$ & $0.2537(10)$ \\
\hline $\mathrm{C} 1 \mathrm{~B}$ & $0.84285(16)$ & $0.38706(18)$ & $0.62055(11)$ & $0.0643(8)$ & $0.761(3)$ \\
\hline $\mathrm{C} 2 \mathrm{~B}$ & $0.86564(17)$ & $0.32722(19)$ & $0.60004(11)$ & $0.0676(9)$ & $0.761(3)$ \\
\hline C3B & 0.8507 (3) & $0.3045(3)$ & $0.55144(14)$ & $0.1096(14)$ & $0.761(3)$ \\
\hline H3B & 0.824550 & 0.323452 & 0.531406 & $0.131 *$ & $0.761(3)$ \\
\hline C4B & $0.8743(6)$ & $0.2535(8)$ & $0.5317(3)$ & $0.139(4)$ & $0.761(3)$ \\
\hline H4B & 0.864501 & 0.239071 & 0.498283 & $0.167 *$ & $0.761(3)$ \\
\hline C5B & $0.9102(6)$ & $0.2251(5)$ & 0.5595 & $0.122(3)$ & $0.761(3)$ \\
\hline H5B & 0.926576 & 0.191734 & 0.545315 & $0.146^{*}$ & $0.761(3)$ \\
\hline C6B & $0.9238(5)$ & $0.2436(6)$ & $0.6083(3)$ & $0.117(3)$ & $0.761(3)$ \\
\hline H6B & 0.947295 & 0.221585 & 0.628218 & $0.140 *$ & $0.761(3)$ \\
\hline C7B & 0.9019 (4) & $0.2963(4)$ & $0.6283(3)$ & 0.0942 (19) & $0.761(3)$ \\
\hline H7B & 0.912232 & 0.310638 & 0.661646 & $0.113 *$ & $0.761(3)$ \\
\hline C8B & $0.90054(18)$ & 0.47139 (19) & $0.60328(12)$ & $0.0690(9)$ & $0.761(3)$ \\
\hline C9B & $0.8767(2)$ & $0.5277(2)$ & $0.59935(15)$ & $0.0889(11)$ & $0.761(3)$ \\
\hline H9B & 0.823725 & 0.512459 & 0.605925 & $0.107 *$ & $0.761(3)$ \\
\hline $\mathrm{C} 10 \mathrm{~B}$ & $0.9287(7)$ & $0.6052(4)$ & $0.5861(3)$ & $0.114(3)$ & $0.761(3)$ \\
\hline $\mathrm{H} 10 \mathrm{~B}$ & 0.910946 & 0.641732 & 0.583345 & $0.137^{*}$ & $0.761(3)$ \\
\hline C11B & $1.0068(7)$ & $0.6283(5)$ & $0.5769(4)$ & $0.135(4)$ & $0.761(3)$ \\
\hline H11B & 1.042807 & 0.681234 & 0.568895 & $0.162 *$ & $0.761(3)$ \\
\hline C12B & $1.0322(4)$ & $0.5740(5)$ & $0.5793(4)$ & $0.149(4)$ & $0.761(3)$ \\
\hline H12B & 1.085032 & 0.589464 & 0.572045 & $0.179 *$ & $0.761(3)$ \\
\hline C13B & $0.9792(3)$ & $0.4963(3)$ & $0.5927(2)$ & 0.1009 (17) & $0.761(3)$ \\
\hline H13B & 0.997098 & 0.459746 & 0.594685 & $0.121 *$ & $0.761(3)$ \\
\hline C14B & $0.8371(2)$ & $0.3825(2)$ & 0.67749 (13) & $0.0613(8)$ & $0.761(3)$ \\
\hline C15B & $0.7720(2)$ & $0.3181(3)$ & $0.69901(15)$ & 0.0925 (12) & $0.761(3)$ \\
\hline H15B & 0.731546 & 0.280294 & 0.678923 & $0.111 *$ & $0.761(3)$ \\
\hline C16B & $0.7663(4)$ & $0.3092(5)$ & 0.7500 & $0.100(2)$ & $0.761(3)$ \\
\hline
\end{tabular}




\begin{tabular}{|c|c|c|c|c|c|}
\hline H16B & 0.722188 & 0.265369 & 0.764060 & $0.120 *$ & $0.761(3)$ \\
\hline C17B & $0.8247(4)$ & $0.3639(4)$ & 0.77990 (19) & 0.0905 (16) & $0.761(3)$ \\
\hline H17B & 0.821027 & 0.357339 & 0.814286 & $0.109 *$ & $0.761(3)$ \\
\hline C18B & $0.8887(3)$ & $0.4284(3)$ & $0.75912(17)$ & $0.0960(15)$ & $0.761(3)$ \\
\hline H18B & 0.929017 & 0.466024 & 0.779317 & $0.115^{*}$ & $0.761(3)$ \\
\hline C19B & $0.8936(3)$ & $0.4378(4)$ & $0.7080(2)$ & $0.0756(15)$ & $0.761(3)$ \\
\hline H19B & 0.936631 & 0.483054 & 0.694220 & $0.091 *$ & $0.761(3)$ \\
\hline $\mathrm{O} 1 \mathrm{C}$ & 0.666667 & 0.333333 & $0.6444(5)$ & $0.096(4)$ & $0.239(3)$ \\
\hline $\mathrm{H} 1 \mathrm{C}$ & 0.682444 & 0.378577 & 0.630954 & $0.144^{*}$ & $0.0797(10)$ \\
\hline $\mathrm{C} 1 \mathrm{C}$ & 0.666667 & 0.333333 & $0.6968(6)$ & $0.067(4)$ & $0.239(3)$ \\
\hline $\mathrm{C} 2 \mathrm{C}$ & $0.7494(7)$ & $0.3565(7)$ & $0.7159(5)$ & $0.086(3)$ & $0.239(3)$ \\
\hline $\mathrm{C} 3 \mathrm{C}$ & $0.7614(16)$ & $0.3248(15)$ & $0.7597(8)$ & $0.124(11)$ & $0.239(3)$ \\
\hline $\mathrm{H} 3 \mathrm{C}$ & 0.718063 & 0.289797 & 0.779120 & $0.148^{*}$ & $0.239(3)$ \\
\hline $\mathrm{C} 4 \mathrm{C}$ & $0.8382(16)$ & $0.3459(18)$ & $0.7740(10)$ & $0.190(16)$ & $0.239(3)$ \\
\hline $\mathrm{H} 4 \mathrm{C}$ & 0.847018 & 0.325127 & 0.803037 & $0.228 *$ & $0.239(3)$ \\
\hline C5C & $0.9014(13)$ & $0.3981(14)$ & 0.7447 (11) & $0.171(11)$ & $0.239(3)$ \\
\hline $\mathrm{H} 5 \mathrm{C}$ & 0.952844 & 0.411478 & 0.754391 & $0.206^{*}$ & $0.239(3)$ \\
\hline $\mathrm{C} 6 \mathrm{C}$ & $0.8922(13)$ & $0.431(2)$ & $0.7026(13)$ & $0.174(16)$ & $0.239(3)$ \\
\hline $\mathrm{H} 6 \mathrm{C}$ & 0.936126 & 0.466658 & 0.683861 & $0.208 *$ & $0.239(3)$ \\
\hline $\mathrm{C} 7 \mathrm{C}$ & $0.8161(8)$ & $0.4091(9)$ & $0.6883(6)$ & $0.105(5)$ & $0.239(3)$ \\
\hline $\mathrm{H} 7 \mathrm{C}$ & 0.808723 & 0.430334 & 0.659071 & $0.126^{*}$ & $0.239(3)$ \\
\hline O1D & $0.7407(5)$ & $0.4307(4)$ & $0.5563(3)$ & $0.104(3)$ & $0.239(3)$ \\
\hline H1DA & 0.735152 & 0.429060 & 0.586798 & $0.156^{*}$ & $0.0797(10)$ \\
\hline H1DB & 0.754328 & 0.398510 & 0.546886 & $0.156^{*}$ & $0.0797(10)$ \\
\hline H1DC & 0.696732 & 0.418001 & 0.543089 & $0.156^{*}$ & $0.0797(10)$ \\
\hline C1D & $0.7984(6)$ & $0.5107(5)$ & $0.5410(4)$ & $0.074(2)$ & $0.239(3)$ \\
\hline C2D & $0.7747(6)$ & $0.5696(6)$ & 0.5642 & $0.074(3)$ & $0.239(3)$ \\
\hline $\mathrm{C} 3 \mathrm{D}$ & $0.7567(9)$ & $0.5648(8)$ & $0.6131(4)$ & $0.122(5)$ & $0.239(3)$ \\
\hline H3D & 0.755580 & 0.524072 & 0.632119 & $0.146^{*}$ & $0.239(3)$ \\
\hline C4D & $0.7398(13)$ & $0.6189(14)$ & $0.6353(10)$ & $0.124(10)$ & $0.239(3)$ \\
\hline $\mathrm{H} 4 \mathrm{D}$ & 0.728498 & 0.614212 & 0.669221 & $0.149 *$ & $0.239(3)$ \\
\hline C5D & $0.7391(18)$ & 0.6777 (17) & 0.6098 (11) & $0.112(8)$ & $0.239(3)$ \\
\hline H5D & 0.728146 & 0.714530 & 0.624757 & $0.134^{*}$ & $0.239(3)$ \\
\hline C6D & $0.756(3)$ & 0.6801 (19) & $0.5606(12)$ & $0.23(2)$ & $0.239(3)$ \\
\hline H6D & 0.760372 & 0.722931 & 0.542143 & $0.270^{*}$ & $0.239(3)$ \\
\hline C7D & $0.766(2)$ & 0.6232 (19) & $0.5360(10)$ & $0.109(10)$ & $0.239(3)$ \\
\hline H7D & 0.766762 & 0.621529 & 0.501386 & $0.130 *$ & $0.239(3)$ \\
\hline C8D & $0.8812(6)$ & $0.5292(6)$ & $0.5580(4)$ & $0.073(3)$ & $0.239(3)$ \\
\hline C9D & $0.9417(15)$ & $0.6018(12)$ & $0.5772(8)$ & $0.120(10)$ & $0.239(3)$ \\
\hline H9D & 0.931304 & 0.643600 & 0.580757 & $0.143 *$ & $0.239(3)$ \\
\hline C10D & $1.0155(15)$ & $0.6146(15)$ & $0.5909(8)$ & $0.105(7)$ & $0.239(3)$ \\
\hline H10D & 1.054059 & 0.664218 & 0.603007 & $0.126^{*}$ & $0.239(3)$ \\
\hline C11D & $1.0319(15)$ & $0.5536(16)$ & $0.5866(10)$ & $0.132(9)$ & $0.239(3)$ \\
\hline H11D & 1.079899 & 0.559758 & 0.598487 & $0.159^{*}$ & $0.239(3)$ \\
\hline C12D & $0.9767(11)$ & $0.4832(13)$ & $0.5647(6)$ & $0.124(7)$ & $0.239(3)$ \\
\hline H12D & 0.990107 & 0.445364 & 0.554674 & $0.149^{*}$ & $0.239(3)$ \\
\hline C13D & $0.9009(8)$ & $0.4708(7)$ & $0.5581(7)$ & $0.113(5)$ & $0.239(3)$ \\
\hline H13D & 0.859930 & 0.418489 & 0.553273 & $0.136^{*}$ & $0.239(3)$ \\
\hline
\end{tabular}




\begin{tabular}{llllll}
$\mathrm{C} 14 \mathrm{D}$ & $0.7968(6)$ & $0.5122(7)$ & $0.4824(4)$ & $0.076(3)$ & $0.239(3)$ \\
$\mathrm{C} 15 \mathrm{D}$ & $0.7274(7)$ & $0.4662(7)$ & $0.4579(5)$ & $0.101(4)$ & $0.239(3)$ \\
$\mathrm{H} 15 \mathrm{D}$ & 0.681858 & 0.431590 & 0.475739 & $0.122^{*}$ & $0.239(3)$ \\
$\mathrm{C} 16 \mathrm{D}$ & $0.7233(16)$ & $0.4698(16)$ & $0.4069(8)$ & $0.109(9)$ & $0.239(3)$ \\
$\mathrm{H} 16 \mathrm{D}$ & 0.675128 & 0.438305 & 0.390548 & $0.131^{*}$ & $0.239(3)$ \\
$\mathrm{C} 17 \mathrm{D}$ & $0.7902(15)$ & $0.5197(17)$ & $0.3806(7)$ & $0.087(6)$ & $0.239(3)$ \\
$\mathrm{H} 17 \mathrm{D}$ & 0.788042 & 0.520924 & 0.345998 & $0.104^{*}$ & $0.239(3)$ \\
$\mathrm{C} 18 \mathrm{D}$ & $0.8597(15)$ & $0.5677(16)$ & $0.4045(7)$ & $0.086(6)$ & $0.239(3)$ \\
$\mathrm{H} 18 \mathrm{D}$ & 0.904107 & 0.605123 & 0.386849 & $0.103^{*}$ & $0.239(3)$ \\
$\mathrm{C} 19 \mathrm{D}$ & $0.8642(12)$ & $0.5605(17)$ & $0.4559(7)$ & $0.102(8)$ & $0.239(3)$ \\
$\mathrm{H} 19 \mathrm{D}$ & 0.913176 & 0.588580 & 0.472064 & $0.122^{*}$ & $0.239(3)$ \\
\hline
\end{tabular}

Atomic displacement parameters $\left(\AA^{2}\right)$

\begin{tabular}{|c|c|c|c|c|c|c|}
\hline & $U^{11}$ & $U^{22}$ & $U^{33}$ & $U^{12}$ & $U^{13}$ & $U^{23}$ \\
\hline O1A & $0.117(2)$ & $0.117(2)$ & $0.040(2)$ & $0.0584(10)$ & 0.000 & 0.000 \\
\hline $\mathrm{C} 1 \mathrm{~A}$ & $0.072(2)$ & $0.072(2)$ & $0.040(2)$ & $0.0362(10)$ & 0.000 & 0.000 \\
\hline $\mathrm{C} 2 \mathrm{~A}$ & $0.0617(19)$ & $0.0650(19)$ & $0.0534(17)$ & $0.0281(16)$ & $0.0058(14)$ & $0.0045(14)$ \\
\hline $\mathrm{C} 3 \mathrm{~A}$ & $0.078(3)$ & $0.091(3)$ & $0.092(3)$ & $0.044(2)$ & $0.014(2)$ & $-0.007(2)$ \\
\hline $\mathrm{C} 4 \mathrm{~A}$ & $0.091(5)$ & $0.117(5)$ & $0.157(7)$ & $0.063(4)$ & $0.027(4)$ & $-0.002(4)$ \\
\hline $\mathrm{C} 5 \mathrm{~A}$ & $0.057(3)$ & $0.103(7)$ & $0.166(8)$ & $0.028(3)$ & $-0.012(4)$ & $0.026(4)$ \\
\hline C6A & $0.081(4)$ & $0.097(7)$ & $0.122(5)$ & $0.037(4)$ & $-0.031(4)$ & $-0.015(4)$ \\
\hline C7A & $0.073(3)$ & $0.091(4)$ & $0.076(3)$ & $0.039(3)$ & $-0.006(3)$ & $-0.008(3)$ \\
\hline O1B & $0.0541(13)$ & $0.131(2)$ & $0.0955(18)$ & $0.0443(14)$ & $-0.0238(11)$ & $-0.0075(14)$ \\
\hline $\mathrm{C} 1 \mathrm{~B}$ & $0.0453(15)$ & $0.083(2)$ & $0.0640(18)$ & $0.0313(15)$ & $-0.0102(13)$ & $-0.0049(15)$ \\
\hline $\mathrm{C} 2 \mathrm{~B}$ & $0.0574(18)$ & $0.077(2)$ & $0.0620(19)$ & $0.0289(17)$ & $-0.0053(14)$ & $-0.0049(15)$ \\
\hline $\mathrm{C} 3 \mathrm{~B}$ & $0.148(4)$ & $0.127(4)$ & $0.075(3)$ & $0.085(3)$ & $-0.015(2)$ & $-0.018(2)$ \\
\hline $\mathrm{C} 4 \mathrm{~B}$ & $0.209(12)$ & $0.162(9)$ & $0.089(4)$ & $0.125(9)$ & $-0.016(5)$ & $-0.031(4)$ \\
\hline $\mathrm{C} 5 \mathrm{~B}$ & $0.162(8)$ & $0.122(4)$ & $0.116(5)$ & $0.096(5)$ & $0.022(5)$ & $-0.013(4)$ \\
\hline $\mathrm{C} 6 \mathrm{~B}$ & $0.149(7)$ & $0.146(8)$ & $0.105(5)$ & $0.111(7)$ & $-0.006(5)$ & $-0.010(5)$ \\
\hline $\mathrm{C} 7 \mathrm{~B}$ & $0.115(4)$ & $0.116(5)$ & $0.081(3)$ & $0.080(4)$ & $-0.013(3)$ & $-0.020(3)$ \\
\hline $\mathrm{C} 8 \mathrm{~B}$ & $0.0591(19)$ & $0.082(2)$ & $0.063(2)$ & $0.0330(17)$ & $-0.0030(15)$ & $0.0023(17)$ \\
\hline $\mathrm{C} 9 \mathrm{~B}$ & $0.097(3)$ & $0.098(3)$ & $0.084(3)$ & $0.059(3)$ & $0.002(2)$ & $0.007(2)$ \\
\hline $\mathrm{C} 10 \mathrm{~B}$ & $0.151(7)$ & $0.106(5)$ & $0.107(4)$ & $0.080(5)$ & $0.014(4)$ & $0.024(3)$ \\
\hline $\mathrm{C} 11 \mathrm{~B}$ & $0.153(9)$ & $0.098(4)$ & $0.128(8)$ & $0.043(5)$ & $0.018(6)$ & $0.034(4)$ \\
\hline $\mathrm{C} 12 \mathrm{~B}$ & $0.098(5)$ & $0.115(5)$ & $0.214(10)$ & $0.038(4)$ & $0.057(5)$ & $0.036(5)$ \\
\hline C13B & $0.069(3)$ & $0.086(3)$ & $0.141(5)$ & $0.033(2)$ & $0.023(3)$ & $0.018(3)$ \\
\hline C14B & $0.050(2)$ & $0.074(2)$ & $0.0629(19)$ & $0.0335(18)$ & $-0.0025(15)$ & $-0.0024(16)$ \\
\hline C15B & $0.071(2)$ & $0.096(3)$ & $0.081(3)$ & $0.020(2)$ & -0.0015 (19) & $-0.009(2)$ \\
\hline C16B & $0.090(4)$ & $0.101(4)$ & $0.078(3)$ & $0.026(3)$ & $0.020(3)$ & $0.011(3)$ \\
\hline C17B & $0.108(5)$ & $0.113(4)$ & $0.056(2)$ & $0.059(3)$ & $0.004(3)$ & $0.002(2)$ \\
\hline C18B & $0.101(3)$ & $0.095(3)$ & $0.073(2)$ & $0.035(3)$ & $-0.022(2)$ & $-0.013(2)$ \\
\hline C19B & $0.066(3)$ & $0.078(3)$ & $0.064(2)$ & $0.022(2)$ & $-0.013(2)$ & $-0.001(2)$ \\
\hline $\mathrm{O} 1 \mathrm{C}$ & $0.112(6)$ & $0.112(6)$ & $0.063(6)$ & $0.056(3)$ & 0.000 & 0.000 \\
\hline $\mathrm{C} 1 \mathrm{C}$ & $0.070(5)$ & $0.070(5)$ & $0.063(7)$ & $0.035(3)$ & 0.000 & 0.000 \\
\hline $\mathrm{C} 2 \mathrm{C}$ & $0.088(6)$ & $0.083(8)$ & $0.086(7)$ & $0.043(6)$ & $-0.017(5)$ & $-0.016(6)$ \\
\hline $\mathrm{C} 3 \mathrm{C}$ & $0.129(13)$ & $0.17(2)$ & $0.103(16)$ & $0.102(16)$ & $-0.009(12)$ & $0.019(16)$ \\
\hline $\mathrm{C} 4 \mathrm{C}$ & $0.132(15)$ & $0.18(3)$ & $0.23(3)$ & $0.059(18)$ & $-0.094(16)$ & $0.00(2)$ \\
\hline
\end{tabular}




$\begin{array}{lllllll}\text { C5C } & 0.090(11) & 0.132(19) & 0.27(3) & 0.035(11) & -0.081(13) & -0.049(16) \\ \text { C6C } & 0.095(12) & 0.21(4) & 0.21(3) & 0.080(19) & -0.018(17) & -0.03(2) \\ \text { C7C } & 0.086(8) & 0.107(11) & 0.127(12) & 0.051(8) & 0.014(8) & 0.020(8) \\ \text { O1D } & 0.091(5) & 0.070(4) & 0.125(7) & 0.021(4) & 0.011(5) & 0.030(4) \\ \text { C1D } & 0.065(5) & 0.051(5) & 0.092(6) & 0.018(4) & 0.005(4) & 0.014(4) \\ \text { C2D } & 0.068(6) & 0.067(6) & 0.075(6) & 0.026(5) & 0.004(5) & 0.015(4) \\ \text { C3D } & 0.163(14) & 0.136(11) & 0.088(7) & 0.091(11) & 0.053(9) & 0.044(7) \\ \text { C4D } & 0.13(2) & 0.15(2) & 0.110(13) & 0.087(19) & 0.037(14) & 0.012(12) \\ \text { C5D } & 0.077(11) & 0.153(19) & 0.130(15) & 0.077(14) & -0.016(10) & -0.013(13) \\ \text { C6D } & 0.43(7) & 0.19(3) & 0.16(2) & 0.24(4) & 0.10(3) & 0.06(2) \\ \text { C7D } & 0.131(19) & 0.12(2) & 0.119(14) & 0.10(2) & 0.049(14) & 0.049(15) \\ \text { C8D } & 0.071(5) & 0.077(6) & 0.071(7) & 0.036(5) & 0.008(5) & 0.010(5) \\ \text { C9D } & 0.084(10) & 0.117(13) & 0.14(2) & 0.041(10) & -0.018(13) & -0.028(14) \\ \text { C10D } & 0.077(9) & 0.142(19) & 0.059(9) & 0.028(12) & -0.004(7) & -0.010(12) \\ \text { C11D } & 0.134(17) & 0.20(2) & 0.081(12) & 0.098(15) & -0.037(13) & 0.005(13) \\ \text { C12D } & 0.135(11) & 0.191(17) & 0.086(12) & 0.111(12) & -0.018(10) & -0.019(11) \\ \text { C13D } & 0.099(8) & 0.087(8) & 0.156(14) & 0.049(7) & -0.002(9) & 0.005(8) \\ \text { C14D } & 0.066(6) & 0.068(7) & 0.091(6) & 0.032(5) & 0.007(4) & 0.010(5) \\ \text { C15D } & 0.088(8) & 0.084(9) & 0.109(8) & 0.026(7) & -0.011(6) & 0.001(7) \\ \text { C16D } & 0.103(13) & 0.12(2) & 0.117(10) & 0.061(13) & -0.034(9) & -0.017(11) \\ \text { C17D } & 0.136(14) & 0.091(12) & 0.063(8) & 0.079(12) & -0.020(7) & -0.031(7) \\ \text { C18D } & 0.088(11) & 0.131(15) & 0.066(8) & 0.075(10) & 0.006(6) & 0.001(7) \\ \text { C19D } & 0.094(10) & 0.13(2) & 0.068(8) & 0.048(10) & 0.012(7) & 0.032(10) \\ & & & & & & \end{array}$

Geometric parameters (A, $\stackrel{\circ}{)}$

\begin{tabular}{llll}
\hline $\mathrm{O} 1 \mathrm{~A}-\mathrm{C} 1 \mathrm{~A}$ & $1.436(5)$ & $\mathrm{O} 1 \mathrm{C}-\mathrm{C} 1 \mathrm{C}$ & $1.407(19)$ \\
$\mathrm{O} 1 \mathrm{~A}-\mathrm{H} 1 \mathrm{~A}$ & 0.8500 & $\mathrm{O} 1 \mathrm{C}-\mathrm{H} 1 \mathrm{C}$ & 0.8500 \\
$\mathrm{O} 1 \mathrm{~A}-\mathrm{H} 1 \mathrm{~A}^{\mathrm{i}}$ & 0.8500 & $\mathrm{O} 1 \mathrm{C}-\mathrm{H} 1 \mathrm{C}^{\mathrm{i}}$ & 0.8501 \\
$\mathrm{O} 1 \mathrm{~A}-\mathrm{H} 1 \mathrm{~A}^{\mathrm{ii}}$ & 0.8501 & $\mathrm{O} 1 \mathrm{C}-\mathrm{H} 1 \mathrm{C}^{\mathrm{ii}}$ & 0.8501 \\
$\mathrm{C} 1 \mathrm{~A}-\mathrm{C} 2 \mathrm{~A}^{\mathrm{i}}$ & $1.526(3)$ & $\mathrm{C} 1 \mathrm{C}-\mathrm{C} 2 \mathrm{C}$ & $1.518(12)$ \\
$\mathrm{C} 1 \mathrm{~A}-\mathrm{C} 2 \mathrm{~A}^{\mathrm{ii}}$ & $1.526(3)$ & $\mathrm{C} 1 \mathrm{C}-\mathrm{C} 2 \mathrm{C}^{\mathrm{ii}}$ & $1.518(12)$ \\
$\mathrm{C} 1 \mathrm{~A}-\mathrm{C} 2 \mathrm{~A}$ & $1.526(3)$ & $\mathrm{C} 1 \mathrm{C}-\mathrm{C} 2 \mathrm{C}^{\mathrm{i}}$ & $1.518(12)$ \\
$\mathrm{C} 2 \mathrm{~A}-\mathrm{C} 7 \mathrm{~A}$ & $1.380(7)$ & $\mathrm{C} 2 \mathrm{C}-\mathrm{C} 7 \mathrm{C}$ & $1.391(14)$ \\
$\mathrm{C} 2 \mathrm{~A}-\mathrm{C} 3 \mathrm{~A}$ & $1.380(5)$ & $\mathrm{C} 2 \mathrm{C}-\mathrm{C} 3 \mathrm{C}$ & $1.399(18)$ \\
$\mathrm{C} 3 \mathrm{~A}-\mathrm{C} 4 \mathrm{~A}$ & $1.383(10)$ & $\mathrm{C} 3 \mathrm{C}-\mathrm{C} 4 \mathrm{C}$ & 0.9300 \\
$\mathrm{C} 3 \mathrm{~A}-\mathrm{H} 3 \mathrm{~A}$ & 0.9300 & $\mathrm{C} 3 \mathrm{C}-\mathrm{H} 3 \mathrm{C}$ & $1.38(2)$ \\
$\mathrm{C} 4 \mathrm{~A}-\mathrm{C} 5 \mathrm{~A}$ & $1.388(11)$ & $\mathrm{C} 4 \mathrm{C}-\mathrm{C} 5 \mathrm{C}$ & 0.9300 \\
$\mathrm{C} 4 \mathrm{~A}-\mathrm{H} 4 \mathrm{~A}$ & 0.9300 & $\mathrm{C} 4 \mathrm{C}-\mathrm{H} 4 \mathrm{C}$ & $1.35(2)$ \\
$\mathrm{C} 5 \mathrm{~A}-\mathrm{C} 6 \mathrm{~A}$ & $1.354(10)$ & $\mathrm{C} 5 \mathrm{C}-\mathrm{C} 6 \mathrm{C}$ & 0.9300 \\
$\mathrm{C} 5 \mathrm{~A}-\mathrm{H} 5 \mathrm{~A}$ & 0.9300 & $\mathrm{C} 5 \mathrm{C}-\mathrm{H} 5 \mathrm{C}$ & $1.368(19)$ \\
$\mathrm{C} 6 \mathrm{~A}-\mathrm{C} 7 \mathrm{~A}$ & $1.371(9)$ & $\mathrm{C} 6 \mathrm{C}-\mathrm{C} 7 \mathrm{C}$ & 0.9300 \\
$\mathrm{C} 6 \mathrm{~A}-\mathrm{H} 6 \mathrm{~A}$ & 0.9300 & $\mathrm{C} 6 \mathrm{C}-\mathrm{H} 6 \mathrm{C}$ & 0.9300 \\
$\mathrm{C} 7 \mathrm{~A}-\mathrm{H} 7 \mathrm{~A}$ & 0.9300 & $\mathrm{C} 7 \mathrm{C}-\mathrm{H} 7 \mathrm{C}$ & $1.442(11)$ \\
$\mathrm{O} 1 \mathrm{C}-\mathrm{C} 1 \mathrm{~B}$ & $1.438(3)$ & O1D-C1D & 0.8254 \\
$\mathrm{O} 1 \mathrm{~B}-\mathrm{H} 1 \mathrm{BA}$ & 0.8172 & O1D-H1DA & 0.8264 \\
O1B-H1BB & 0.8171 & O1D-H1DB & 0.8364 \\
O1B-H1BC & 0.8543 & O1D-H1DC
\end{tabular}




\begin{tabular}{|c|c|c|c|}
\hline $\mathrm{C} 1 \mathrm{~B}-\mathrm{C} 8 \mathrm{~B}$ & $1.516(4)$ & $\mathrm{C} 1 \mathrm{D}-\mathrm{C} 8 \mathrm{D}$ & $1.524(14)$ \\
\hline $\mathrm{C} 1 \mathrm{~B}-\mathrm{C} 2 \mathrm{~B}$ & $1.531(4)$ & $\mathrm{C} 1 \mathrm{D}-\mathrm{C} 2 \mathrm{D}$ & $1.554(14)$ \\
\hline $\mathrm{C} 1 \mathrm{~B}-\mathrm{C} 14 \mathrm{~B}$ & $1.532(4)$ & $\mathrm{C} 1 \mathrm{D}-\mathrm{C} 14 \mathrm{D}$ & $1.575(15)$ \\
\hline $\mathrm{C} 2 \mathrm{~B}-\mathrm{C} 7 \mathrm{~B}$ & $1.357(7)$ & $\mathrm{C} 2 \mathrm{D}-\mathrm{C} 3 \mathrm{D}$ & $1.350(12)$ \\
\hline $\mathrm{C} 2 \mathrm{~B}-\mathrm{C} 3 \mathrm{~B}$ & $1.361(4)$ & $\mathrm{C} 2 \mathrm{D}-\mathrm{C} 7 \mathrm{D}$ & $1.361(19)$ \\
\hline $\mathrm{C} 3 \mathrm{~B}-\mathrm{C} 4 \mathrm{~B}$ & $1.382(12)$ & $\mathrm{C} 3 \mathrm{D}-\mathrm{C} 4 \mathrm{D}$ & $1.38(2)$ \\
\hline $\mathrm{C} 3 \mathrm{~B}-\mathrm{H} 3 \mathrm{~B}$ & 0.9300 & $\mathrm{C} 3 \mathrm{D}-\mathrm{H} 3 \mathrm{D}$ & 0.9300 \\
\hline $\mathrm{C} 4 \mathrm{~B}-\mathrm{C} 5 \mathrm{~B}$ & $1.312(11)$ & $\mathrm{C} 4 \mathrm{D}-\mathrm{C} 5 \mathrm{D}$ & $1.33(2)$ \\
\hline $\mathrm{C} 4 \mathrm{~B}-\mathrm{H} 4 \mathrm{~B}$ & 0.9300 & $\mathrm{C} 4 \mathrm{D}-\mathrm{H} 4 \mathrm{D}$ & 0.9300 \\
\hline $\mathrm{C} 5 \mathrm{~B}-\mathrm{C} 6 \mathrm{~B}$ & $1.351(7)$ & $\mathrm{C} 5 \mathrm{D}-\mathrm{C} 6 \mathrm{D}$ & $1.355(17)$ \\
\hline $\mathrm{C} 5 \mathrm{~B}-\mathrm{H} 5 \mathrm{~B}$ & 0.9300 & $\mathrm{C} 5 \mathrm{D}-\mathrm{H} 5 \mathrm{D}$ & 0.9300 \\
\hline $\mathrm{C} 6 \mathrm{~B}-\mathrm{C} 7 \mathrm{~B}$ & $1.392(9)$ & $\mathrm{C} 6 \mathrm{D}-\mathrm{C} 7 \mathrm{D}$ & $1.38(2)$ \\
\hline $\mathrm{C} 6 \mathrm{~B}-\mathrm{H} 6 \mathrm{~B}$ & 0.9300 & $\mathrm{C} 6 \mathrm{D}-\mathrm{H} 6 \mathrm{D}$ & 0.9300 \\
\hline $\mathrm{C} 7 \mathrm{~B}-\mathrm{H} 7 \mathrm{~B}$ & 0.9300 & C7D-H7D & 0.9300 \\
\hline $\mathrm{C} 8 \mathrm{~B}-\mathrm{C} 13 \mathrm{~B}$ & $1.376(5)$ & $\mathrm{C} 8 \mathrm{D}-\mathrm{C} 13 \mathrm{D}$ & $1.360(13)$ \\
\hline $\mathrm{C} 8 \mathrm{~B}-\mathrm{C} 9 \mathrm{~B}$ & $1.381(5)$ & $C 8 D-C 9 D$ & $1.402(18)$ \\
\hline $\mathrm{C} 9 \mathrm{~B}-\mathrm{C} 10 \mathrm{~B}$ & $1.370(9)$ & $\mathrm{C} 9 \mathrm{D}-\mathrm{C} 10 \mathrm{D}$ & $1.37(2)$ \\
\hline $\mathrm{C} 9 \mathrm{~B}-\mathrm{H} 9 \mathrm{~B}$ & 0.9300 & C9D-H9D & 0.9300 \\
\hline $\mathrm{C} 10 \mathrm{~B}-\mathrm{C} 11 \mathrm{~B}$ & $1.366(11)$ & $\mathrm{C} 10 \mathrm{D}-\mathrm{C} 11 \mathrm{D}$ & $1.37(2)$ \\
\hline $\mathrm{C} 10 \mathrm{~B}-\mathrm{H} 10 \mathrm{~B}$ & 0.9300 & C10D-H10D & 0.9300 \\
\hline $\mathrm{C} 11 \mathrm{~B}-\mathrm{C} 12 \mathrm{~B}$ & $1.364(9)$ & $\mathrm{C} 11 \mathrm{D}-\mathrm{C} 12 \mathrm{D}$ & $1.37(2)$ \\
\hline $\mathrm{C} 11 \mathrm{~B}-\mathrm{H} 11 \mathrm{~B}$ & 0.9300 & C11D-H11D & 0.9300 \\
\hline $\mathrm{C} 12 \mathrm{~B}-\mathrm{C} 13 \mathrm{~B}$ & $1.378(9)$ & $\mathrm{C} 12 \mathrm{D}-\mathrm{C} 13 \mathrm{D}$ & $1.372(16)$ \\
\hline $\mathrm{C} 12 \mathrm{~B}-\mathrm{H} 12 \mathrm{~B}$ & 0.9300 & $\mathrm{C} 12 \mathrm{D}-\mathrm{H} 12 \mathrm{D}$ & 0.9300 \\
\hline $\mathrm{C} 13 \mathrm{~B}-\mathrm{H} 13 \mathrm{~B}$ & 0.9300 & $\mathrm{C} 13 \mathrm{D}-\mathrm{H} 13 \mathrm{D}$ & 0.9300 \\
\hline $\mathrm{C} 14 \mathrm{~B}-\mathrm{C} 19 \mathrm{~B}$ & $1.356(7)$ & $\mathrm{C} 14 \mathrm{D}-\mathrm{C} 15 \mathrm{D}$ & $1.353(13)$ \\
\hline $\mathrm{C} 14 \mathrm{~B}-\mathrm{C} 15 \mathrm{~B}$ & $1.379(5)$ & $\mathrm{C} 14 \mathrm{D}-\mathrm{C} 19 \mathrm{D}$ & $1.365(18)$ \\
\hline $\mathrm{C} 15 \mathrm{~B}-\mathrm{C} 16 \mathrm{~B}$ & $1.378(8)$ & $\mathrm{C} 15 \mathrm{D}-\mathrm{C} 16 \mathrm{D}$ & $1.374(19)$ \\
\hline $\mathrm{C} 15 \mathrm{~B}-\mathrm{H} 15 \mathrm{~B}$ & 0.9300 & $\mathrm{C} 15 \mathrm{D}-\mathrm{H} 15 \mathrm{D}$ & 0.9300 \\
\hline $\mathrm{C} 16 \mathrm{~B}-\mathrm{C} 17 \mathrm{~B}$ & $1.357(9)$ & $\mathrm{C} 16 \mathrm{D}-\mathrm{C} 17 \mathrm{D}$ & $1.36(2)$ \\
\hline $\mathrm{C} 16 \mathrm{~B}-\mathrm{H} 16 \mathrm{~B}$ & 0.9300 & $\mathrm{C} 16 \mathrm{D}-\mathrm{H} 16 \mathrm{D}$ & 0.9300 \\
\hline $\mathrm{C} 17 \mathrm{~B}-\mathrm{C} 18 \mathrm{~B}$ & $1.361(7)$ & $\mathrm{C} 17 \mathrm{D}-\mathrm{C} 18 \mathrm{D}$ & $1.355(19)$ \\
\hline $\mathrm{C} 17 \mathrm{~B}-\mathrm{H} 17 \mathrm{~B}$ & 0.9300 & $\mathrm{C} 17 \mathrm{D}-\mathrm{H} 17 \mathrm{D}$ & 0.9300 \\
\hline $\mathrm{C} 18 \mathrm{~B}-\mathrm{C} 19 \mathrm{~B}$ & $1.382(7)$ & $\mathrm{C} 18 \mathrm{D}-\mathrm{C} 19 \mathrm{D}$ & $1.393(18)$ \\
\hline $\mathrm{C} 18 \mathrm{~B}-\mathrm{H} 18 \mathrm{~B}$ & 0.9300 & $\mathrm{C} 18 \mathrm{D}-\mathrm{H} 18 \mathrm{D}$ & 0.9300 \\
\hline C19B-H19B & 0.9300 & C19D-H19D & 0.9300 \\
\hline $\mathrm{C} 1 \mathrm{~A}-\mathrm{O} 1 \mathrm{~A}-\mathrm{H} 1 \mathrm{~A}$ & 112.8 & $\mathrm{C} 14 \mathrm{~B}-\mathrm{C} 19 \mathrm{~B}-\mathrm{H} 19 \mathrm{~B}$ & 119.3 \\
\hline $\mathrm{C} 1 \mathrm{~A}-\mathrm{O} 1 \mathrm{~A}-\mathrm{H} 1 \mathrm{~A}^{\mathrm{i}}$ & $112.764(1)$ & $\mathrm{C} 18 \mathrm{~B}-\mathrm{C} 19 \mathrm{~B}-\mathrm{H} 19 \mathrm{~B}$ & 119.3 \\
\hline $\mathrm{C} 1 \mathrm{~A}-\mathrm{O} 1 \mathrm{~A}-\mathrm{H} 1 \mathrm{~A}^{\mathrm{ii}}$ & $112.760(1)$ & $\mathrm{C} 1 \mathrm{C}-\mathrm{O} 1 \mathrm{C}-\mathrm{H} 1 \mathrm{C}$ & 115.2 \\
\hline $\mathrm{H} 1 \mathrm{~A}-\mathrm{O} 1 \mathrm{~A}-\mathrm{H} 1 \mathrm{~A}^{\mathrm{i}}$ & 106.0 & $\mathrm{C} 1 \mathrm{C}-\mathrm{O} 1 \mathrm{C}-\mathrm{H} 1 \mathrm{C}^{\mathrm{i}}$ & $115.240(4)$ \\
\hline $\mathrm{H} 1 \mathrm{~A}-\mathrm{O} 1 \mathrm{~A}-\mathrm{H} 1 \mathrm{~A}^{\mathrm{ii}}$ & 106.0 & $\mathrm{H} 1 \mathrm{C}-\mathrm{O} 1 \mathrm{C}-\mathrm{H} 1 \mathrm{C}^{\mathrm{i}}$ & 103.1 \\
\hline $\mathrm{H} 1 \mathrm{~A}^{\mathrm{i}}-\mathrm{O} 1 \mathrm{~A}-\mathrm{H} 1 \mathrm{~A}^{\mathrm{ii}}$ & 106.0 & $\mathrm{O} 1 \mathrm{C}-\mathrm{C} 1 \mathrm{C}-\mathrm{C} 2 \mathrm{C}$ & $109.7(7)$ \\
\hline $\mathrm{O} 1 \mathrm{~A}-\mathrm{C} 1 \mathrm{~A}-\mathrm{C} 2 \mathrm{~A}^{\mathrm{i}}$ & $108.00(19)$ & $\mathrm{O} 1 \mathrm{C}-\mathrm{C} 1 \mathrm{C}-\mathrm{C} 2 \mathrm{C}^{\mathrm{ii}}$ & $109.7(7)$ \\
\hline $\mathrm{O} 1 \mathrm{~A}-\mathrm{C} 1 \mathrm{~A}-\mathrm{C} 2 \mathrm{~A}^{\mathrm{ii}}$ & $108.00(19)$ & $\mathrm{C} 2 \mathrm{C}-\mathrm{C} 1 \mathrm{C}-\mathrm{C} 2 \mathrm{C}^{\mathrm{ii}}$ & $109.3(8)$ \\
\hline $\mathrm{C} 2 \mathrm{~A}^{\mathrm{i}}-\mathrm{C} 1 \mathrm{~A}-\mathrm{C} 2 \mathrm{~A}^{\mathrm{ii}}$ & $110.91(18)$ & $\mathrm{O} 1 \mathrm{C}-\mathrm{C} 1 \mathrm{C}-\mathrm{C} 2 \mathrm{C}^{\mathrm{i}}$ & $109.7(7)$ \\
\hline $\mathrm{O} 1 \mathrm{~A}-\mathrm{C} 1 \mathrm{~A}-\mathrm{C} 2 \mathrm{~A}$ & $108.00(19)$ & $\mathrm{C} 2 \mathrm{C}-\mathrm{C} 1 \mathrm{C}-\mathrm{C} 2 \mathrm{C}^{\mathrm{i}}$ & $109.3(8)$ \\
\hline $\mathrm{C} 2 \mathrm{~A}-\mathrm{C} 1 \mathrm{~A}-\mathrm{C} 2 \mathrm{~A}$ & $110.91(18)$ & $\mathrm{C} 2 \mathrm{C}^{\mathrm{ii}}-\mathrm{C} 1 \mathrm{C}-\mathrm{C} 2 \mathrm{C}^{\mathrm{i}}$ & $109.3(8)$ \\
\hline
\end{tabular}




\begin{tabular}{|c|c|}
\hline $\mathrm{C} 2 \mathrm{~A}^{\mathrm{ii}}-\mathrm{C} 1 \mathrm{~A}-\mathrm{C} 2 \mathrm{~A}$ & $110.90(18)$ \\
\hline $\mathrm{C} 7 \mathrm{~A}-\mathrm{C} 2 \mathrm{~A}-\mathrm{C} 3 \mathrm{~A}$ & $117.9(4)$ \\
\hline $\mathrm{C} 7 \mathrm{~A}-\mathrm{C} 2 \mathrm{~A}-\mathrm{C} 1 \mathrm{~A}$ & $122.2(4)$ \\
\hline $\mathrm{C} 3 \mathrm{~A}-\mathrm{C} 2 \mathrm{~A}-\mathrm{C} 1 \mathrm{~A}$ & $119.8(3)$ \\
\hline $\mathrm{C} 2 \mathrm{~A}-\mathrm{C} 3 \mathrm{~A}-\mathrm{C} 4 \mathrm{~A}$ & $120.6(5)$ \\
\hline $\mathrm{C} 2 \mathrm{~A}-\mathrm{C} 3 \mathrm{~A}-\mathrm{H} 3 \mathrm{~A}$ & 119.7 \\
\hline $\mathrm{C} 4 \mathrm{~A}-\mathrm{C} 3 \mathrm{~A}-\mathrm{H} 3 \mathrm{~A}$ & 119.7 \\
\hline $\mathrm{C} 3 \mathrm{~A}-\mathrm{C} 4 \mathrm{~A}-\mathrm{C} 5 \mathrm{~A}$ & $119.4(7)$ \\
\hline $\mathrm{C} 3 \mathrm{~A}-\mathrm{C} 4 \mathrm{~A}-\mathrm{H} 4 \mathrm{~A}$ & 120.3 \\
\hline $\mathrm{C} 5 \mathrm{~A}-\mathrm{C} 4 \mathrm{~A}-\mathrm{H} 4 \mathrm{~A}$ & 120.3 \\
\hline $\mathrm{C} 6 \mathrm{~A}-\mathrm{C} 5 \mathrm{~A}-\mathrm{C} 4 \mathrm{~A}$ & $120.7(7)$ \\
\hline $\mathrm{C} 6 \mathrm{~A}-\mathrm{C} 5 \mathrm{~A}-\mathrm{H} 5 \mathrm{~A}$ & 119.6 \\
\hline $\mathrm{C} 4 \mathrm{~A}-\mathrm{C} 5 \mathrm{~A}-\mathrm{H} 5 \mathrm{~A}$ & 119.6 \\
\hline $\mathrm{C} 5 \mathrm{~A}-\mathrm{C} 6 \mathrm{~A}-\mathrm{C} 7 \mathrm{~A}$ & $119.1(8)$ \\
\hline $\mathrm{C} 5 \mathrm{~A}-\mathrm{C} 6 \mathrm{~A}-\mathrm{H} 6 \mathrm{~A}$ & 120.5 \\
\hline $\mathrm{C} 7 \mathrm{~A}-\mathrm{C} 6 \mathrm{~A}-\mathrm{H} 6 \mathrm{~A}$ & 120.5 \\
\hline $\mathrm{C} 6 \mathrm{~A}-\mathrm{C} 7 \mathrm{~A}-\mathrm{C} 2 \mathrm{~A}$ & $122.3(7)$ \\
\hline $\mathrm{C} 6 \mathrm{~A}-\mathrm{C} 7 \mathrm{~A}-\mathrm{H} 7 \mathrm{~A}$ & 118.9 \\
\hline $\mathrm{C} 2 \mathrm{~A}-\mathrm{C} 7 \mathrm{~A}-\mathrm{H} 7 \mathrm{~A}$ & 118.9 \\
\hline $\mathrm{C} 1 \mathrm{~B}-\mathrm{O} 1 \mathrm{~B}-\mathrm{H} 1 \mathrm{BA}$ & 114.2 \\
\hline $\mathrm{C} 1 \mathrm{~B}-\mathrm{O} 1 \mathrm{~B}-\mathrm{H} 1 \mathrm{BB}$ & 110.4 \\
\hline $\mathrm{C} 1 \mathrm{~B}-\mathrm{O} 1 \mathrm{~B}-\mathrm{H} 1 \mathrm{BC}$ & 103.6 \\
\hline $\mathrm{H} 1 \mathrm{BA}-\mathrm{O} 1 \mathrm{~B}-\mathrm{H} 1 \mathrm{BB}$ & 114.5 \\
\hline $\mathrm{H} 1 \mathrm{BA}-\mathrm{O} 1 \mathrm{~B}-\mathrm{H} 1 \mathrm{BC}$ & 106.3 \\
\hline $\mathrm{H} 1 \mathrm{BB}-\mathrm{O} 1 \mathrm{~B}-\mathrm{H} 1 \mathrm{BC}$ & 106.9 \\
\hline $\mathrm{C} 1 \mathrm{~B}-\mathrm{O} 1 \mathrm{~B}-\mathrm{H} 1 \mathrm{C}^{\mathrm{ii}}$ & $119.5(4)$ \\
\hline $\mathrm{O} 1 \mathrm{~B}-\mathrm{C} 1 \mathrm{~B}-\mathrm{C} 8 \mathrm{~B}$ & $107.6(2)$ \\
\hline $\mathrm{O} 1 \mathrm{~B}-\mathrm{C} 1 \mathrm{~B}-\mathrm{C} 2 \mathrm{~B}$ & $107.8(2)$ \\
\hline $\mathrm{C} 8 \mathrm{~B}-\mathrm{C} 1 \mathrm{~B}-\mathrm{C} 2 \mathrm{~B}$ & $111.6(2)$ \\
\hline $\mathrm{O} 1 \mathrm{~B}-\mathrm{C} 1 \mathrm{~B}-\mathrm{C} 14 \mathrm{~B}$ & $107.7(2)$ \\
\hline $\mathrm{C} 8 \mathrm{~B}-\mathrm{C} 1 \mathrm{~B}-\mathrm{C} 14 \mathrm{~B}$ & $111.1(3)$ \\
\hline $\mathrm{C} 2 \mathrm{~B}-\mathrm{C} 1 \mathrm{~B}-\mathrm{C} 14 \mathrm{~B}$ & $110.8(3)$ \\
\hline $\mathrm{C} 7 \mathrm{~B}-\mathrm{C} 2 \mathrm{~B}-\mathrm{C} 3 \mathrm{~B}$ & $117.8(4)$ \\
\hline $\mathrm{C} 7 \mathrm{~B}-\mathrm{C} 2 \mathrm{~B}-\mathrm{C} 1 \mathrm{~B}$ & $122.6(4)$ \\
\hline $\mathrm{C} 3 \mathrm{~B}-\mathrm{C} 2 \mathrm{~B}-\mathrm{C} 1 \mathrm{~B}$ & $119.6(3)$ \\
\hline $\mathrm{C} 2 \mathrm{~B}-\mathrm{C} 3 \mathrm{~B}-\mathrm{C} 4 \mathrm{~B}$ & $120.4(5)$ \\
\hline $\mathrm{C} 2 \mathrm{~B}-\mathrm{C} 3 \mathrm{~B}-\mathrm{H} 3 \mathrm{~B}$ & 119.8 \\
\hline $\mathrm{C} 4 \mathrm{~B}-\mathrm{C} 3 \mathrm{~B}-\mathrm{H} 3 \mathrm{~B}$ & 119.8 \\
\hline $\mathrm{C} 5 \mathrm{~B}-\mathrm{C} 4 \mathrm{~B}-\mathrm{C} 3 \mathrm{~B}$ & $120.8(7)$ \\
\hline $\mathrm{C} 5 \mathrm{~B}-\mathrm{C} 4 \mathrm{~B}-\mathrm{H} 4 \mathrm{~B}$ & 119.6 \\
\hline $\mathrm{C} 3 \mathrm{~B}-\mathrm{C} 4 \mathrm{~B}-\mathrm{H} 4 \mathrm{~B}$ & 119.6 \\
\hline $\mathrm{C} 4 \mathrm{~B}-\mathrm{C} 5 \mathrm{~B}-\mathrm{C} 6 \mathrm{~B}$ & $121.0(8)$ \\
\hline $\mathrm{C} 4 \mathrm{~B}-\mathrm{C} 5 \mathrm{~B}-\mathrm{H} 5 \mathrm{~B}$ & 119.5 \\
\hline $\mathrm{C} 6 \mathrm{~B}-\mathrm{C} 5 \mathrm{~B}-\mathrm{H} 5 \mathrm{~B}$ & 119.5 \\
\hline $\mathrm{C} 5 \mathrm{~B}-\mathrm{C} 6 \mathrm{~B}-\mathrm{C} 7 \mathrm{~B}$ & $118.5(7)$ \\
\hline $\mathrm{C} 5 \mathrm{~B}-\mathrm{C} 6 \mathrm{~B}-\mathrm{H} 6 \mathrm{~B}$ & 120.8 \\
\hline $\mathrm{C} 7 \mathrm{~B}-\mathrm{C} 6 \mathrm{~B}-\mathrm{H} 6 \mathrm{~B}$ & 120.8 \\
\hline $\mathrm{C} 2 \mathrm{~B}-\mathrm{C} 7 \mathrm{~B}-\mathrm{C} 6 \mathrm{~B}$ & $121.4(6)$ \\
\hline
\end{tabular}

\begin{tabular}{|c|c|}
\hline $\mathrm{C} 7 \mathrm{C}-\mathrm{C} 2 \mathrm{C}-\mathrm{C} 3 \mathrm{C}$ & $118.1(14)$ \\
\hline $\mathrm{C} 7 \mathrm{C}-\mathrm{C} 2 \mathrm{C}-\mathrm{C} 1 \mathrm{C}$ & $119.4(11)$ \\
\hline $\mathrm{C} 3 \mathrm{C}-\mathrm{C} 2 \mathrm{C}-\mathrm{C} 1 \mathrm{C}$ & $122.4(14)$ \\
\hline $\mathrm{C} 4 \mathrm{C}-\mathrm{C} 3 \mathrm{C}-\mathrm{C} 2 \mathrm{C}$ & $119.6(19)$ \\
\hline $\mathrm{C} 4 \mathrm{C}-\mathrm{C} 3 \mathrm{C}-\mathrm{H} 3 \mathrm{C}$ & 120.2 \\
\hline $\mathrm{C} 2 \mathrm{C}-\mathrm{C} 3 \mathrm{C}-\mathrm{H} 3 \mathrm{C}$ & 120.2 \\
\hline $\mathrm{C} 5 \mathrm{C}-\mathrm{C} 4 \mathrm{C}-\mathrm{C} 3 \mathrm{C}$ & $119(2)$ \\
\hline $\mathrm{C} 5 \mathrm{C}-\mathrm{C} 4 \mathrm{C}-\mathrm{H} 4 \mathrm{C}$ & 120.5 \\
\hline $\mathrm{C} 3 \mathrm{C}-\mathrm{C} 4 \mathrm{C}-\mathrm{H} 4 \mathrm{C}$ & 120.5 \\
\hline $\mathrm{C} 6 \mathrm{C}-\mathrm{C} 5 \mathrm{C}-\mathrm{C} 4 \mathrm{C}$ & $123.1(19)$ \\
\hline $\mathrm{C} 6 \mathrm{C}-\mathrm{C} 5 \mathrm{C}-\mathrm{H} 5 \mathrm{C}$ & 118.5 \\
\hline $\mathrm{C} 4 \mathrm{C}-\mathrm{C} 5 \mathrm{C}-\mathrm{H} 5 \mathrm{C}$ & 118.5 \\
\hline $\mathrm{C} 5 \mathrm{C}-\mathrm{C} 6 \mathrm{C}-\mathrm{C} 7 \mathrm{C}$ & $118(2)$ \\
\hline $\mathrm{C} 5 \mathrm{C}-\mathrm{C} 6 \mathrm{C}-\mathrm{H} 6 \mathrm{C}$ & 121.2 \\
\hline $\mathrm{C} 7 \mathrm{C}-\mathrm{C} 6 \mathrm{C}-\mathrm{H} 6 \mathrm{C}$ & 121.2 \\
\hline $\mathrm{C} 6 \mathrm{C}-\mathrm{C} 7 \mathrm{C}-\mathrm{C} 2 \mathrm{C}$ & $122.5(16)$ \\
\hline $\mathrm{C} 6 \mathrm{C}-\mathrm{C} 7 \mathrm{C}-\mathrm{H} 7 \mathrm{C}$ & 118.7 \\
\hline $\mathrm{C} 2 \mathrm{C}-\mathrm{C} 7 \mathrm{C}-\mathrm{H} 7 \mathrm{C}$ & 118.7 \\
\hline $\mathrm{C} 1 \mathrm{D}-\mathrm{O} 1 \mathrm{D}-\mathrm{H} 1 \mathrm{DA}$ & 109.8 \\
\hline $\mathrm{C} 1 \mathrm{D}-\mathrm{O} 1 \mathrm{D}-\mathrm{H} 1 \mathrm{DB}$ & 110.7 \\
\hline $\mathrm{C} 1 \mathrm{D}-\mathrm{O} 1 \mathrm{D}-\mathrm{H} 1 \mathrm{DC}$ & 108.8 \\
\hline $\mathrm{H} 1 \mathrm{DA}-\mathrm{O} 1 \mathrm{D}-\mathrm{H} 1 \mathrm{DB}$ & 111.0 \\
\hline H1DA-O1D-H1DC & 108.4 \\
\hline $\mathrm{H} 1 \mathrm{DB}-\mathrm{O} 1 \mathrm{D}-\mathrm{H} 1 \mathrm{DC}$ & 108.1 \\
\hline $\mathrm{O} 1 \mathrm{D}-\mathrm{C} 1 \mathrm{D}-\mathrm{C} 8 \mathrm{D}$ & $109.0(8)$ \\
\hline $\mathrm{O} 1 \mathrm{D}-\mathrm{C} 1 \mathrm{D}-\mathrm{C} 2 \mathrm{D}$ & $108.6(8)$ \\
\hline $\mathrm{C} 8 \mathrm{D}-\mathrm{C} 1 \mathrm{D}-\mathrm{C} 2 \mathrm{D}$ & $111.6(8)$ \\
\hline $\mathrm{O} 1 \mathrm{D}-\mathrm{C} 1 \mathrm{D}-\mathrm{C} 14 \mathrm{D}$ & $107.0(8)$ \\
\hline $\mathrm{C} 8 \mathrm{D}-\mathrm{C} 1 \mathrm{D}-\mathrm{C} 14 \mathrm{D}$ & $108.9(8)$ \\
\hline $\mathrm{C} 2 \mathrm{D}-\mathrm{C} 1 \mathrm{D}-\mathrm{C} 14 \mathrm{D}$ & $111.7(8)$ \\
\hline $\mathrm{C} 3 \mathrm{D}-\mathrm{C} 2 \mathrm{D}-\mathrm{C} 7 \mathrm{D}$ & $117.8(14)$ \\
\hline $\mathrm{C} 3 \mathrm{D}-\mathrm{C} 2 \mathrm{D}-\mathrm{C} 1 \mathrm{D}$ & $120.0(9)$ \\
\hline $\mathrm{C} 7 \mathrm{D}-\mathrm{C} 2 \mathrm{D}-\mathrm{C} 1 \mathrm{D}$ & $122.1(13)$ \\
\hline $\mathrm{C} 2 \mathrm{D}-\mathrm{C} 3 \mathrm{D}-\mathrm{C} 4 \mathrm{D}$ & $121.4(15)$ \\
\hline $\mathrm{C} 2 \mathrm{D}-\mathrm{C} 3 \mathrm{D}-\mathrm{H} 3 \mathrm{D}$ & 119.3 \\
\hline $\mathrm{C} 4 \mathrm{D}-\mathrm{C} 3 \mathrm{D}-\mathrm{H} 3 \mathrm{D}$ & 119.3 \\
\hline $\mathrm{C} 5 \mathrm{D}-\mathrm{C} 4 \mathrm{D}-\mathrm{C} 3 \mathrm{D}$ & $122(2)$ \\
\hline $\mathrm{C} 5 \mathrm{D}-\mathrm{C} 4 \mathrm{D}-\mathrm{H} 4 \mathrm{D}$ & 119.0 \\
\hline $\mathrm{C} 3 \mathrm{D}-\mathrm{C} 4 \mathrm{D}-\mathrm{H} 4 \mathrm{D}$ & 119.0 \\
\hline $\mathrm{C} 4 \mathrm{D}-\mathrm{C} 5 \mathrm{D}-\mathrm{C} 6 \mathrm{D}$ & $115(2)$ \\
\hline $\mathrm{C} 4 \mathrm{D}-\mathrm{C} 5 \mathrm{D}-\mathrm{H} 5 \mathrm{D}$ & 122.3 \\
\hline $\mathrm{C} 6 \mathrm{D}-\mathrm{C} 5 \mathrm{D}-\mathrm{H} 5 \mathrm{D}$ & 122.3 \\
\hline $\mathrm{C} 5 \mathrm{D}-\mathrm{C} 6 \mathrm{D}-\mathrm{C} 7 \mathrm{D}$ & $124(2)$ \\
\hline $\mathrm{C} 5 \mathrm{D}-\mathrm{C} 6 \mathrm{D}-\mathrm{H} 6 \mathrm{D}$ & 117.8 \\
\hline $\mathrm{C} 7 \mathrm{D}-\mathrm{C} 6 \mathrm{D}-\mathrm{H} 6 \mathrm{D}$ & 117.8 \\
\hline $\mathrm{C} 2 \mathrm{D}-\mathrm{C} 7 \mathrm{D}-\mathrm{C} 6 \mathrm{D}$ & $118(2)$ \\
\hline $\mathrm{C} 2 \mathrm{D}-\mathrm{C} 7 \mathrm{D}-\mathrm{H} 7 \mathrm{D}$ & 121.2 \\
\hline $\mathrm{C} 6 \mathrm{D}-\mathrm{C} 7 \mathrm{D}-\mathrm{H} 7 \mathrm{D}$ & 121.2 \\
\hline
\end{tabular}




\begin{tabular}{|c|c|}
\hline $\mathrm{C} 2 \mathrm{~B}-\mathrm{C} 7 \mathrm{~B}-\mathrm{H} 7 \mathrm{~B}$ & 119.3 \\
\hline $\mathrm{C} 6 \mathrm{~B}-\mathrm{C} 7 \mathrm{~B}-\mathrm{H} 7 \mathrm{~B}$ & 119.3 \\
\hline $\mathrm{C} 13 \mathrm{~B}-\mathrm{C} 8 \mathrm{~B}-\mathrm{C} 9 \mathrm{~B}$ & $117.0(4)$ \\
\hline $\mathrm{C} 13 \mathrm{~B}-\mathrm{C} 8 \mathrm{~B}-\mathrm{C} 1 \mathrm{~B}$ & $122.0(3)$ \\
\hline $\mathrm{C} 9 \mathrm{~B}-\mathrm{C} 8 \mathrm{~B}-\mathrm{C} 1 \mathrm{~B}$ & $121.0(3)$ \\
\hline $\mathrm{C} 10 \mathrm{~B}-\mathrm{C} 9 \mathrm{~B}-\mathrm{C} 8 \mathrm{~B}$ & $122.0(6)$ \\
\hline $\mathrm{C} 10 \mathrm{~B}-\mathrm{C} 9 \mathrm{~B}-\mathrm{H} 9 \mathrm{~B}$ & 119.0 \\
\hline $\mathrm{C} 8 \mathrm{~B}-\mathrm{C} 9 \mathrm{~B}-\mathrm{H} 9 \mathrm{~B}$ & 119.0 \\
\hline $\mathrm{C} 11 \mathrm{~B}-\mathrm{C} 10 \mathrm{~B}-\mathrm{C} 9 \mathrm{~B}$ & $119.4(8)$ \\
\hline $\mathrm{C} 11 \mathrm{~B}-\mathrm{C} 10 \mathrm{~B}-\mathrm{H} 10 \mathrm{~B}$ & 120.3 \\
\hline $\mathrm{C} 9 \mathrm{~B}-\mathrm{C} 10 \mathrm{~B}-\mathrm{H} 10 \mathrm{~B}$ & 120.3 \\
\hline $\mathrm{C} 12 \mathrm{~B}-\mathrm{C} 11 \mathrm{~B}-\mathrm{C} 10 \mathrm{~B}$ & $120.2(8)$ \\
\hline $\mathrm{C} 12 \mathrm{~B}-\mathrm{C} 11 \mathrm{~B}-\mathrm{H} 11 \mathrm{~B}$ & 119.9 \\
\hline $\mathrm{C} 10 \mathrm{~B}-\mathrm{C} 11 \mathrm{~B}-\mathrm{H} 11 \mathrm{~B}$ & 119.9 \\
\hline $\mathrm{C} 11 \mathrm{~B}-\mathrm{C} 12 \mathrm{~B}-\mathrm{C} 13 \mathrm{~B}$ & $119.6(7)$ \\
\hline $\mathrm{C} 11 \mathrm{~B}-\mathrm{C} 12 \mathrm{~B}-\mathrm{H} 12 \mathrm{~B}$ & 120.2 \\
\hline $\mathrm{C} 13 \mathrm{~B}-\mathrm{C} 12 \mathrm{~B}-\mathrm{H} 12 \mathrm{~B}$ & 120.2 \\
\hline $\mathrm{C} 8 \mathrm{~B}-\mathrm{C} 13 \mathrm{~B}-\mathrm{C} 12 \mathrm{~B}$ & $121.6(5)$ \\
\hline $\mathrm{C} 8 \mathrm{~B}-\mathrm{C} 13 \mathrm{~B}-\mathrm{H} 13 \mathrm{~B}$ & 119.2 \\
\hline $\mathrm{C} 12 \mathrm{~B}-\mathrm{C} 13 \mathrm{~B}-\mathrm{H} 13 \mathrm{~B}$ & 119.2 \\
\hline $\mathrm{C} 19 \mathrm{~B}-\mathrm{C} 14 \mathrm{~B}-\mathrm{C} 15 \mathrm{~B}$ & $118.1(4)$ \\
\hline $\mathrm{C} 19 \mathrm{~B}-\mathrm{C} 14 \mathrm{~B}-\mathrm{C} 1 \mathrm{~B}$ & $123.4(3)$ \\
\hline $\mathrm{C} 15 \mathrm{~B}-\mathrm{C} 14 \mathrm{~B}-\mathrm{C} 1 \mathrm{~B}$ & $118.5(3)$ \\
\hline $\mathrm{C} 16 \mathrm{~B}-\mathrm{C} 15 \mathrm{~B}-\mathrm{C} 14 \mathrm{~B}$ & $120.6(4)$ \\
\hline $\mathrm{C} 16 \mathrm{~B}-\mathrm{C} 15 \mathrm{~B}-\mathrm{H} 15 \mathrm{~B}$ & 119.7 \\
\hline $\mathrm{C} 14 \mathrm{~B}-\mathrm{C} 15 \mathrm{~B}-\mathrm{H} 15 \mathrm{~B}$ & 119.7 \\
\hline $\mathrm{C} 17 \mathrm{~B}-\mathrm{C} 16 \mathrm{~B}-\mathrm{C} 15 \mathrm{~B}$ & $120.5(5)$ \\
\hline $\mathrm{C} 17 \mathrm{~B}-\mathrm{C} 16 \mathrm{~B}-\mathrm{H} 16 \mathrm{~B}$ & 119.8 \\
\hline $\mathrm{C} 15 \mathrm{~B}-\mathrm{C} 16 \mathrm{~B}-\mathrm{H} 16 \mathrm{~B}$ & 119.8 \\
\hline $\mathrm{C} 16 \mathrm{~B}-\mathrm{C} 17 \mathrm{~B}-\mathrm{C} 18 \mathrm{~B}$ & $119.5(5)$ \\
\hline $\mathrm{C} 16 \mathrm{~B}-\mathrm{C} 17 \mathrm{~B}-\mathrm{H} 17 \mathrm{~B}$ & 120.3 \\
\hline $\mathrm{C} 18 \mathrm{~B}-\mathrm{C} 17 \mathrm{~B}-\mathrm{H} 17 \mathrm{~B}$ & 120.3 \\
\hline $\mathrm{C} 17 \mathrm{~B}-\mathrm{C} 18 \mathrm{~B}-\mathrm{C} 19 \mathrm{~B}$ & $120.0(5)$ \\
\hline $\mathrm{C} 17 \mathrm{~B}-\mathrm{C} 18 \mathrm{~B}-\mathrm{H} 18 \mathrm{~B}$ & 120.0 \\
\hline $\mathrm{C} 19 \mathrm{~B}-\mathrm{C} 18 \mathrm{~B}-\mathrm{H} 18 \mathrm{~B}$ & 120.0 \\
\hline $\mathrm{C} 14 \mathrm{~B}-\mathrm{C} 19 \mathrm{~B}-\mathrm{C} 18 \mathrm{~B}$ & $121.3(4)$ \\
\hline $\mathrm{O} 1 \mathrm{~A}-\mathrm{C} 1 \mathrm{~A}-\mathrm{C} 2 \mathrm{~A}-\mathrm{C} 7 \mathrm{~A}$ & $-145.6(5)$ \\
\hline $\mathrm{C} 2 \mathrm{~A}-\mathrm{C} 1 \mathrm{~A}-\mathrm{C} 2 \mathrm{~A}-\mathrm{C} 7 \mathrm{~A}$ & $-27.4(6)$ \\
\hline $\mathrm{C} 2 \mathrm{~A}^{\mathrm{ii}}-\mathrm{C} 1 \mathrm{~A}-\mathrm{C} 2 \mathrm{~A}-\mathrm{C} 7 \mathrm{~A}$ & $96.3(5)$ \\
\hline $\mathrm{O} 1 \mathrm{~A}-\mathrm{C} 1 \mathrm{~A}-\mathrm{C} 2 \mathrm{~A}-\mathrm{C} 3 \mathrm{~A}$ & $36.8(3)$ \\
\hline $\mathrm{C} 2 \mathrm{~A}^{\mathrm{i}}-\mathrm{C} 1 \mathrm{~A}-\mathrm{C} 2 \mathrm{~A}-\mathrm{C} 3 \mathrm{~A}$ & $155.0(3)$ \\
\hline $\mathrm{C} 2 \mathrm{~A}^{\mathrm{ii}}-\mathrm{C} 1 \mathrm{~A}-\mathrm{C} 2 \mathrm{~A}-\mathrm{C} 3 \mathrm{~A}$ & $-81.3(5)$ \\
\hline $\mathrm{C} 7 \mathrm{~A}-\mathrm{C} 2 \mathrm{~A}-\mathrm{C} 3 \mathrm{~A}-\mathrm{C} 4 \mathrm{~A}$ & $-0.2(8)$ \\
\hline $\mathrm{C} 1 \mathrm{~A}-\mathrm{C} 2 \mathrm{~A}-\mathrm{C} 3 \mathrm{~A}-\mathrm{C} 4 \mathrm{~A}$ & $177.5(6)$ \\
\hline $\mathrm{C} 2 \mathrm{~A}-\mathrm{C} 3 \mathrm{~A}-\mathrm{C} 4 \mathrm{~A}-\mathrm{C} 5 \mathrm{~A}$ & $-0.9(11)$ \\
\hline $\mathrm{C} 3 \mathrm{~A}-\mathrm{C} 4 \mathrm{~A}-\mathrm{C} 5 \mathrm{~A}-\mathrm{C} 6 \mathrm{~A}$ & $1.5(14)$ \\
\hline $\mathrm{C} 4 \mathrm{~A}-\mathrm{C} 5 \mathrm{~A}-\mathrm{C} 6 \mathrm{~A}-\mathrm{C} 7 \mathrm{~A}$ & $-1.1(14)$ \\
\hline
\end{tabular}

$\begin{array}{ll}\text { C13D-C8D-C9D } & 113.0(14) \\ \text { C13D-C8D-C1D } & 120.2(10) \\ \text { C9D-C8D-C1D } & 126.7(14) \\ \text { C10D-C9D-C8D } & 124(2) \\ \text { C10D-C9D-H9D } & 118.2 \\ \text { C8D-C9D-H9D } & 118.2 \\ \text { C9D-C10D-C11D } & 119(2) \\ \text { C9D-C10D-H10D } & 120.4 \\ \text { C11D-C10D-H10D } & 120.4 \\ \text { C10D-C11D-C12D } & 119.2(19) \\ \text { C10D-C11D-H11D } & 120.4 \\ \text { C12D-C11D-H11D } & 120.4 \\ \text { C13D-C12D-C11D } & 117.5(17) \\ \text { C13D-C12D-H12D } & 121.2 \\ \text { C11D-C12D-H12D } & 121.2 \\ \text { C8D-C13D-C12D } & 124.9(14) \\ \text { C8D-C13D-H13D } & 117.5 \\ \text { C12D-C13D-H13D } & 117.5 \\ \text { C15D-C14D-C19D } & 119.3(13) \\ \text { C15D-C14D-C1D } & 119.9(10) \\ \text { C19D-C14D-C1D } & 120.9(11) \\ \text { C14D-C15D-C16D } & 121.0(15) \\ \text { C14D-C15D-H15D } & 119.5 \\ \text { C16D-C15D-H15D } & 119.5 \\ \text { C17D-C16D-C15D } & 120(2) \\ \text { C17D-C16D-H16D } & 120.2 \\ \text { C15D-C16D-H16D } & 120.2 \\ \text { C18D-C17D-C16D } & 120.2(18) \\ \text { C18D-C17D-H17D } & 119.9 \\ \text { C16D-C17D-H17D } & 119.9 \\ \text { C17D-C18D-C19D } & 119.5(18) \\ \text { C17D-C18D-H18D } & 120.2 \\ \text { C19D-C18D-H18D } & 120.2 \\ \text { C14D-C19D-C18D } & 119.9(18) \\ \text { C14D-C19D-H19D } & 120.0 \\ \text { C18D-C19D-H19D } & 120.0 \\ & \\ \text { O1C-C1C-C2C-C7C } & 31.7(13) \\ \text { C2C Ci-C1C-C2C-C7C } & 151.9(13) \\ \text { C2C-C1C-C2C-C7C } & -88.6(18) \\ \text { O1C-C1C-C2C-C3C } & -146.3(13) \\ \text { C2CC-C1C-C2C-C3C } & -26.0(18) \\ \text { C2C-C1C-C2C-C3C } & 93.5(14) \\ \text { C7C-C2C-C3C-C4C } & -0.5(17) \\ \text { C1C-C2C-C3C-C4C } & 177.4(15) \\ \text { C2C-C3C-C4C-C5C } & 0(2) \\ \text { C3C-C4C-C5C-C6C } & 1(4) \\ \text { C4C-C5C-C6C-C7C } & -1(5) \\ & \end{array}$




\begin{tabular}{|c|c|}
\hline $\mathrm{C} 5 \mathrm{~A}-\mathrm{C} 6 \mathrm{~A}-\mathrm{C} 7 \mathrm{~A}-\mathrm{C} 2 \mathrm{~A}$ & $0.0(13)$ \\
\hline $\mathrm{C} 3 \mathrm{~A}-\mathrm{C} 2 \mathrm{~A}-\mathrm{C} 7 \mathrm{~A}-\mathrm{C} 6 \mathrm{~A}$ & $0.6(10)$ \\
\hline $\mathrm{C} 1 \mathrm{~A}-\mathrm{C} 2 \mathrm{~A}-\mathrm{C} 7 \mathrm{~A}-\mathrm{C} 6 \mathrm{~A}$ & $-177.0(6)$ \\
\hline $\mathrm{O} 1 \mathrm{~B}-\mathrm{C} 1 \mathrm{~B}-\mathrm{C} 2 \mathrm{~B}-\mathrm{C} 7 \mathrm{~B}$ & $-138.5(4)$ \\
\hline $\mathrm{C} 8 \mathrm{~B}-\mathrm{C} 1 \mathrm{~B}-\mathrm{C} 2 \mathrm{~B}-\mathrm{C} 7 \mathrm{~B}$ & $103.5(5)$ \\
\hline $\mathrm{C} 14 \mathrm{~B}-\mathrm{C} 1 \mathrm{~B}-\mathrm{C} 2 \mathrm{~B}-\mathrm{C} 7 \mathrm{~B}$ & $-20.9(5)$ \\
\hline $\mathrm{O} 1 \mathrm{~B}-\mathrm{C} 1 \mathrm{~B}-\mathrm{C} 2 \mathrm{~B}-\mathrm{C} 3 \mathrm{~B}$ & $42.6(4)$ \\
\hline $\mathrm{C} 8 \mathrm{~B}-\mathrm{C} 1 \mathrm{~B}-\mathrm{C} 2 \mathrm{~B}-\mathrm{C} 3 \mathrm{~B}$ & $-75.3(4)$ \\
\hline $\mathrm{C} 14 \mathrm{~B}-\mathrm{C} 1 \mathrm{~B}-\mathrm{C} 2 \mathrm{~B}-\mathrm{C} 3 \mathrm{~B}$ & $160.3(3)$ \\
\hline $\mathrm{C} 7 \mathrm{~B}-\mathrm{C} 2 \mathrm{~B}-\mathrm{C} 3 \mathrm{~B}-\mathrm{C} 4 \mathrm{~B}$ & $-1.9(8)$ \\
\hline $\mathrm{C} 1 \mathrm{~B}-\mathrm{C} 2 \mathrm{~B}-\mathrm{C} 3 \mathrm{~B}-\mathrm{C} 4 \mathrm{~B}$ & $177.0(6)$ \\
\hline $\mathrm{C} 2 \mathrm{~B}-\mathrm{C} 3 \mathrm{~B}-\mathrm{C} 4 \mathrm{~B}-\mathrm{C} 5 \mathrm{~B}$ & $1.0(13)$ \\
\hline $\mathrm{C} 3 \mathrm{~B}-\mathrm{C} 4 \mathrm{~B}-\mathrm{C} 5 \mathrm{~B}-\mathrm{C} 6 \mathrm{~B}$ & $1.3(16)$ \\
\hline $\mathrm{C} 4 \mathrm{~B}-\mathrm{C} 5 \mathrm{~B}-\mathrm{C} 6 \mathrm{~B}-\mathrm{C} 7 \mathrm{~B}$ & $-2.7(14)$ \\
\hline $\mathrm{C} 3 \mathrm{~B}-\mathrm{C} 2 \mathrm{~B}-\mathrm{C} 7 \mathrm{~B}-\mathrm{C} 6 \mathrm{~B}$ & $0.5(9)$ \\
\hline $\mathrm{C} 1 \mathrm{~B}-\mathrm{C} 2 \mathrm{~B}-\mathrm{C} 7 \mathrm{~B}-\mathrm{C} 6 \mathrm{~B}$ & $-178.3(6)$ \\
\hline $\mathrm{C} 5 \mathrm{~B}-\mathrm{C} 6 \mathrm{~B}-\mathrm{C} 7 \mathrm{~B}-\mathrm{C} 2 \mathrm{~B}$ & $1.7(12)$ \\
\hline $\mathrm{O} 1 \mathrm{~B}-\mathrm{C} 1 \mathrm{~B}-\mathrm{C} 8 \mathrm{~B}-\mathrm{C} 13 \mathrm{~B}$ & $-147.2(4)$ \\
\hline $\mathrm{C} 2 \mathrm{~B}-\mathrm{C} 1 \mathrm{~B}-\mathrm{C} 8 \mathrm{~B}-\mathrm{C} 13 \mathrm{~B}$ & $-29.1(5)$ \\
\hline $\mathrm{C} 14 \mathrm{~B}-\mathrm{C} 1 \mathrm{~B}-\mathrm{C} 8 \mathrm{~B}-\mathrm{C} 13 \mathrm{~B}$ & $95.1(4)$ \\
\hline $\mathrm{O} 1 \mathrm{~B}-\mathrm{C} 1 \mathrm{~B}-\mathrm{C} 8 \mathrm{~B}-\mathrm{C} 9 \mathrm{~B}$ & $35.4(4)$ \\
\hline $\mathrm{C} 2 \mathrm{~B}-\mathrm{C} 1 \mathrm{~B}-\mathrm{C} 8 \mathrm{~B}-\mathrm{C} 9 \mathrm{~B}$ & $153.5(3)$ \\
\hline $\mathrm{C} 14 \mathrm{~B}-\mathrm{C} 1 \mathrm{~B}-\mathrm{C} 8 \mathrm{~B}-\mathrm{C} 9 \mathrm{~B}$ & $-82.3(4)$ \\
\hline $\mathrm{C} 13 \mathrm{~B}-\mathrm{C} 8 \mathrm{~B}-\mathrm{C} 9 \mathrm{~B}-\mathrm{C} 10 \mathrm{~B}$ & $-0.6(7)$ \\
\hline $\mathrm{C} 1 \mathrm{~B}-\mathrm{C} 8 \mathrm{~B}-\mathrm{C} 9 \mathrm{~B}-\mathrm{C} 10 \mathrm{~B}$ & $176.9(5)$ \\
\hline $\mathrm{C} 8 \mathrm{~B}-\mathrm{C} 9 \mathrm{~B}-\mathrm{C} 10 \mathrm{~B}-\mathrm{C} 11 \mathrm{~B}$ & $-0.7(10)$ \\
\hline $\mathrm{C} 9 \mathrm{~B}-\mathrm{C} 10 \mathrm{~B}-\mathrm{C} 11 \mathrm{~B}-\mathrm{C} 12 \mathrm{~B}$ & $2.1(14)$ \\
\hline $\mathrm{C} 10 \mathrm{~B}-\mathrm{C} 11 \mathrm{~B}-\mathrm{C} 12 \mathrm{~B}-\mathrm{C} 13 \mathrm{~B}$ & $-2.1(15)$ \\
\hline $\mathrm{C} 9 \mathrm{~B}-\mathrm{C} 8 \mathrm{~B}-\mathrm{C} 13 \mathrm{~B}-\mathrm{C} 12 \mathrm{~B}$ & $0.7(9)$ \\
\hline $\mathrm{C} 1 \mathrm{~B}-\mathrm{C} 8 \mathrm{~B}-\mathrm{C} 13 \mathrm{~B}-\mathrm{C} 12 \mathrm{~B}$ & $-176.9(6)$ \\
\hline $\mathrm{C} 11 \mathrm{~B}-\mathrm{C} 12 \mathrm{~B}-\mathrm{C} 13 \mathrm{~B}-\mathrm{C} 8 \mathrm{~B}$ & $0.7(13)$ \\
\hline $\mathrm{O} 1 \mathrm{~B}-\mathrm{C} 1 \mathrm{~B}-\mathrm{C} 14 \mathrm{~B}-\mathrm{C} 19 \mathrm{~B}$ & $-137.9(5)$ \\
\hline $\mathrm{C} 8 \mathrm{~B}-\mathrm{C} 1 \mathrm{~B}-\mathrm{C} 14 \mathrm{~B}-\mathrm{C} 19 \mathrm{~B}$ & $-20.2(6)$ \\
\hline $\mathrm{C} 2 \mathrm{~B}-\mathrm{C} 1 \mathrm{~B}-\mathrm{C} 14 \mathrm{~B}-\mathrm{C} 19 \mathrm{~B}$ & $104.4(5)$ \\
\hline $\mathrm{O} 1 \mathrm{~B}-\mathrm{C} 1 \mathrm{~B}-\mathrm{C} 14 \mathrm{~B}-\mathrm{C} 15 \mathrm{~B}$ & $43.6(4)$ \\
\hline $\mathrm{C} 8 \mathrm{~B}-\mathrm{C} 1 \mathrm{~B}-\mathrm{C} 14 \mathrm{~B}-\mathrm{C} 15 \mathrm{~B}$ & $161.2(3)$ \\
\hline $\mathrm{C} 2 \mathrm{~B}-\mathrm{C} 1 \mathrm{~B}-\mathrm{C} 14 \mathrm{~B}-\mathrm{C} 15 \mathrm{~B}$ & $-74.2(4)$ \\
\hline $\mathrm{C} 19 \mathrm{~B}-\mathrm{C} 14 \mathrm{~B}-\mathrm{C} 15 \mathrm{~B}-\mathrm{C} 16 \mathrm{~B}$ & $-2.2(8)$ \\
\hline $\mathrm{C} 1 \mathrm{~B}-\mathrm{C} 14 \mathrm{~B}-\mathrm{C} 15 \mathrm{~B}-\mathrm{C} 16 \mathrm{~B}$ & $176.5(5)$ \\
\hline $\mathrm{C} 14 \mathrm{~B}-\mathrm{C} 15 \mathrm{~B}-\mathrm{C} 16 \mathrm{~B}-\mathrm{C} 17 \mathrm{~B}$ & $0.2(10)$ \\
\hline $\mathrm{C} 15 \mathrm{~B}-\mathrm{C} 16 \mathrm{~B}-\mathrm{C} 17 \mathrm{~B}-\mathrm{C} 18 \mathrm{~B}$ & $0.8(11)$ \\
\hline $\mathrm{C} 16 \mathrm{~B}-\mathrm{C} 17 \mathrm{~B}-\mathrm{C} 18 \mathrm{~B}-\mathrm{C} 19 \mathrm{~B}$ & $0.2(9)$ \\
\hline $\mathrm{C} 15 \mathrm{~B}-\mathrm{C} 14 \mathrm{~B}-\mathrm{C} 19 \mathrm{~B}-\mathrm{C} 18 \mathrm{~B}$ & $3.2(8)$ \\
\hline $\mathrm{C} 1 \mathrm{~B}-\mathrm{C} 14 \mathrm{~B}-\mathrm{C} 19 \mathrm{~B}-\mathrm{C} 18 \mathrm{~B}$ & $-175.4(5)$ \\
\hline $\mathrm{C} 17 \mathrm{~B}-\mathrm{C} 18 \mathrm{~B}-\mathrm{C} 19 \mathrm{~B}-\mathrm{C} 14 \mathrm{~B}$ & $-2.3(9)$ \\
\hline
\end{tabular}

$\begin{array}{ll}\text { C5C-C6C-C7C-C2C } & 1(5) \\ \text { C3C-C2C-C7C-C6C } & 0(3) \\ \text { C1C-C2C-C7C-C6C } & -178(3) \\ \text { O1D-C1D-C2D-C3D } & 48.5(13) \\ \text { C8D-C1D-C2D-C3D } & -71.6(13) \\ \text { C14D-C1D-C2D-C3D } & 166.2(10) \\ \text { O1D-C1D-C2D-C7D } & -127(2) \\ \text { C8D-C1D-C2D-C7D } & 113(2) \\ \text { C14D-C1D-C2D-C7D } & -9(2) \\ \text { C7D-C2D-C3D-C4D } & -8(2) \\ \text { C1D-C2D-C3D-C4D } & 176.3(12) \\ \text { C2D-C3D-C4D-C5D } & 1(2) \\ \text { C3D-C4D-C5D-C6D } & 0(4) \\ \text { C4D-C5D-C6D-C7D } & 6(6) \\ \text { C3D-C2D-C7D-C6D } & 13(4) \\ \text { C1D-C2D-C7D-C6D } & -171(3) \\ \text { C5D-C6D-C7D-C2D } & -13(6) \\ \text { O1D-C1D-C8D-C13D } & 35.4(15) \\ \text { C2D-C1D-C8D-C13D } & 155.3(11) \\ \text { C14D-C1D-C8D-C13D } & -80.9(14) \\ \text { O1D-C1D-C8D-C9D } & -140.8(13) \\ \text { C2D-C1D-C8D-C9D } & -20.8(16) \\ \text { C14D-C1D-C8D-C9D } & 102.9(14) \\ \text { C13D-C8D-C9D-C10D } & 4.8(16) \\ \text { C1D-C8D-C9D-C10D } & -178.8(13) \\ \text { C8D-C9D-C10D-C11D } & -1(2) \\ \text { C9D-C10D-C11D-C12D } & 6(3) \\ \text { C10D-C11D-C12D-C13D } & -14(3) \\ \text { C9D-C8D-C13D-C12D } & -15(2) \\ \text { C1D-C8D-C13D-C12D } & 168.8(15) \\ \text { C11D-C12D-C13D-C8D } & 20(3) \\ \text { O1D-C1D-C14D-C15D } & 33.3(14) \\ \text { C8D-C1D-C14D-C15D } & 151.0(11) \\ \text { C2D-C1D-C14D-C15D } & -85.3(12) \\ \text { O1D-C1D-C14D-C19D } & -147.8(18) \\ \text { C8D-C1D-C14D-C19D } & -30.2(19) \\ \text { C2D-C1D-C14D-C19D } & 93.5(19) \\ \text { C19D-C14D-C15D-C16D } & -2.6(19) \\ \text { C1D-C14D-C15D-C16D } & 176.2(14) \\ \text { C14D-C15D-C16D-C17D } & 1(2) \\ \text { C15D-C16D-C17D-C18D } & -2(4) \\ \text { C16D-C17D-C18D-C19D } & 6(4) \\ \text { C15D-C14D-C19D-C18D } & 6(4) \\ \text { C1D-C14D-C19D-C18D } & -173(2) \\ \text { C17D-C18D-C19D-C14D } & -8(4) \\ & \end{array}$

Symmetry codes: (i) $-y+1, x-y, z$; (ii) $-x+y+1,-x+1, z$. 
Hydrogen-bond geometry $\left(\AA,{ }^{\circ}\right)$

\begin{tabular}{lllll}
\hline$D-\mathrm{H} \cdots A$ & $D-\mathrm{H}$ & $\mathrm{H} \cdots A$ & $D \cdots A$ & $D-\mathrm{H} \cdots A$ \\
\hline $\mathrm{O} 1 A-\mathrm{H} 1 A \cdots \mathrm{O} 1 B$ & 0.85 & 2.56 & $2.917(3)$ & 106 \\
$\mathrm{O} 1 B-\mathrm{H} 1 B A \cdots \mathrm{O} 1 A$ & 0.82 & 2.27 & $2.917(3)$ & 136 \\
$\mathrm{O} 1 B-\mathrm{H} 1 B B \cdots \mathrm{O} 1 B^{\mathrm{i}}$ & 0.82 & 2.31 & $2.912(3)$ & 131 \\
$\mathrm{O} 1 B-\mathrm{H} 1 B C \cdots \mathrm{O} 1 B^{\mathrm{ii}}$ & 0.85 & 2.31 & $2.912(4)$ & 128 \\
$\mathrm{O} 1 C-\mathrm{H} 1 C \cdots \mathrm{O} 1 D$ & 0.85 & 2.27 & $2.916(13)$ & 133 \\
$\mathrm{O} 1 D-\mathrm{H} 1 D A \cdots \mathrm{O} 1 C$ & 0.83 & 2.26 & $2.916(13)$ & 136 \\
$\mathrm{O} 1 D-\mathrm{H} 1 D B \cdots \mathrm{O} 1 D^{\mathrm{ii}}$ & 0.83 & 2.35 & $2.947(13)$ & 130 \\
$\mathrm{O} 1 D-\mathrm{H} 1 D C \cdots \mathrm{O} 1 D^{\mathrm{i}}$ & 0.84 & 2.33 & $2.947(13)$ & 132
\end{tabular}

Symmetry codes: (i) $-y+1, x-y, z$; (ii) $-x+y+1,-x+1, z$.

Comparison between the three X-ray structures of triphenylmethanol determined at room temperature

\begin{tabular}{llll}
\hline Date of publication & $1992^{\mathrm{a}}$ & $1999^{\mathrm{b}}$ & $2019^{\mathrm{c}}$ \\
Diffractometer & CAD-4 & R-Axis II & Stadivari \\
Detector & NaI scintillator & Image plate & HPAD $^{\mathrm{d}}$ \\
$T(\mathrm{~K})$ & 294 & 293 & 295 \\
No. independent reflections & 2467 & 3448 & 4523 \\
Refined parameters & 253 & 322 & 483 \\
Data resolution $(\AA)$ & 0.89 & 0.82 & 0.77 \\
Range for $\sigma(\mathrm{C}-\mathrm{C})$ & $0.040-0.007 \AA$ & - & $0.020-0.003 \AA$
\end{tabular}

Nores and references: (a) Ferguson et al. (1992); (b) Serrano-González et al. (1999); (c) this work; (d) Hybrid Pixel Array Detector. 